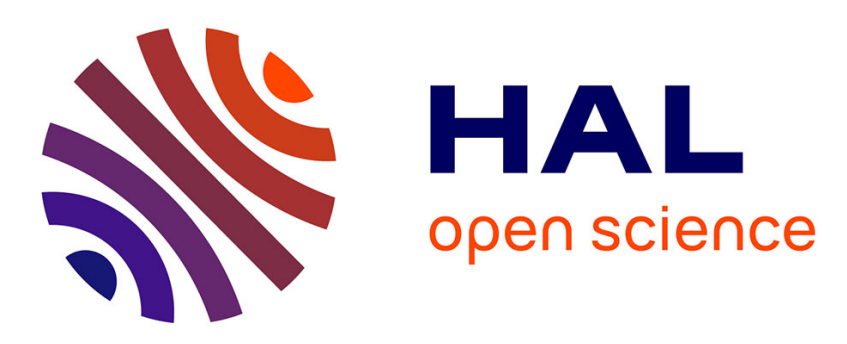

\title{
Shape Optimization of a Coupled Thermal Fluid-Structure Problem in a Level Set Mesh Evolution Framework
}

\author{
Florian Feppon, Grégoire Allaire, Felipe Bordeu, Julien Cortial, Charles
}

Dapogny

\section{To cite this version:}

Florian Feppon, Grégoire Allaire, Felipe Bordeu, Julien Cortial, Charles Dapogny. Shape Optimization of a Coupled Thermal Fluid-Structure Problem in a Level Set Mesh Evolution Framework. SeMA Journal: Boletin de la Sociedad Española de Matemática Aplicada, 2019, 76 (3), pp.413-458. 10.1007/s40324-018-00185-4 . hal-01686770v3

\section{HAL Id: hal-01686770 \\ https://hal.science/hal-01686770v3}

Submitted on 9 Jan 2019

HAL is a multi-disciplinary open access archive for the deposit and dissemination of scientific research documents, whether they are published or not. The documents may come from teaching and research institutions in France or abroad, or from public or private research centers.
L'archive ouverte pluridisciplinaire HAL, est destinée au dépôt et à la diffusion de documents scientifiques de niveau recherche, publiés ou non, émanant des établissements d'enseignement et de recherche français ou étrangers, des laboratoires publics ou privés. 


\title{
SHAPE OPTIMIZATION OF A COUPLED THERMAL FLUID-STRUCTURE PROBLEM IN A LEVEL SET MESH EVOLUTION FRAMEWORK
}

\author{
F. FEPPON ${ }^{12}$, G. ALLAIRE ${ }^{1}$, F. BORDEU ${ }^{2}$, J. CORTIAL ${ }^{2}$ AND C. DAPOGNY ${ }^{3}$ \\ ${ }^{1}$ Centre de Mathématiques Appliquées, École polytechnique, Palaiseau, France \\ ${ }^{2}$ Safran Tech, Magny-les-Hameaux, France \\ ${ }^{3}$ Univ. Grenoble Alpes, CNRS, Inria, Grenoble INP ${ }^{1}$, LJK, 38000 Grenoble, France
}

\begin{abstract}
Hadamard's method of shape differentiation is applied to topology optimization of a weakly coupled three physics problem. The coupling is weak because the equations involved are solved consecutively, namely the steady state Navier-Stokes equations for the fluid domain, first, the convection diffusion equation for the whole domain, second, and the linear thermo-elasticity system in the solid domain, third. Shape sensitivities are derived in a fully Lagrangian setting which allows us to obtain shape derivatives of general objective functions. An emphasis is given on the derivation of the adjoint interface condition dual to the one of equality of the normal stresses at the fluid solid interface. The arguments allowing to obtain this surprising condition are specifically detailed on a simplified scalar problem. Numerical test cases are presented using the level set mesh evolution framework of [4]. It is demonstrated how the implementation enables to treat a variety of shape optimization problems.
\end{abstract}

keywords. Topology and shape optimization, adjoint methods, fluid structure interaction, convective heat transfer, adaptive remeshing.

AMS Subject classifications. 74P10, 76B75, 74F10, 35Q79, 65N50.

\section{Contents}

1. Introduction 2

2. Setting of the three-physic problem 4

3. A simplified scalar fluid structure interaction problem 5

3.1. Presentation of the simplified problem $\quad 6$

3.2. Hadamard's boundary variation method for shape optimization $\quad 7$

3.3. Calculating the shape derivative of a particular objective functional and its adjoint system with Céa's method 8

3.4. A fully Lagrangian setting for computing shape derivatives of arbitrary objective functionals 10

4. Shape derivatives for the three-physic problem 15

5. Numerical representation of shapes and their deformations 19

5.1. Two complementary numerical representations of shapes 20

5.2. Switching between both representations of shapes 21

5.3. The shape optimization algorithm 22

6. Numerical examples 23

6.1. Details about the numerical implementation 23

6.2. A steady-state fluid-structure interaction problem 24

6.3. Convective heat transfer $\quad 27$

6.4. Optimization of a compliant thermoelastic solid with fluid-structure interaction 30

Appendix A. Proof of Proposition 4

$\begin{array}{ll}\text { References } & 37\end{array}$

Corresponding author. Email: florian.feppon@polytechnique.edu.

${ }^{1}$ Institute of Engineering Univ. Grenoble Alpes 


\section{INTRODUCTION}

In this article, we investigate shape and topology optimization problems for a weakly coupled model of heat propagation, fluid flow and structure deformation. The ultimate goal is to optimize mechanical structures subjected to thermal loads and cooled down by a fluid. In such a case, very often, the mechanical deformations and displacements are small. This implies that the fluid domain is fixed at first order, that is, independent of the deformation of the structure. It is therefore natural to consider only a weak coupling between the three physics at stake. First, the hydrodynamic model is solved in the fluid domain (we consider the incompressible Stokes or Navier-Stokes equations). Second, we solve the convection-diffusion equation for the temperature in the whole domain (the reunion of the fluid and solid subdomains). Third, we solve the linearized thermoelasticity system for the description of the solid domain: the forces are a combination of the applied external loads, of the fluid stress applied on the fluid-solid interface at play, and of the thermal dilation induced by the temperature. This weak coupling is of course a major simplification and it dramatically reduces the computational cost since no monolithic coupled system has to be solved. All these equations are considered in a steady-state setting. We refer to Section 2 for more details about our weakly coupled model.

A typical shape optimization problem arising in this context involves an objective function, depending on the geometries of the fluid and solid subdomains, which has to be minimized under some constraints (e.g. volume or mass constraints). This objective function depends on the solution of the weakly coupled model. Its derivative with respect to the geometry of the interface between the fluid and solid domains is computed by Hadamard's method of shape differentiation [32, 40, 39, 51], introducing adjoint states. As far as these adjoint states are concerned, it turns out, classically, that the ordering of the coupling is reversed for the adjoint system: for the direct problem, one first solves the fluid equation, and in a second step the solid equation; for the adjoint problem, it is the opposite, namely elasticity is solved first, followed by fluid mechanics. In addition, a quite surprising mathematical phenomenon occurs: while the fluid and solid equations are coupled by a one-sided interface transmission condition of Neumann type (which accounts for the force exerted by the fluid on the solid), the adjoint equations are, on the contrary, coupled by a Dirichlet interface condition (for details, see Remark 11 or Section 3.3 for the elliptic simplified setting). This 'transformation' of the Neumann interface condition for the direct problem into a Dirichlet interface condition for the adjoint seems new to the best of our knowledge. Since this phenomenon is original and could be hidden in the complexity of our coupled model of heat propagation, fluid flow and structure deformation, for pedagogical reasons, we explain it by using a simplified model at first. This simplified model, exposed in Section 3, is made of two Laplace equations - see (3.1) and (3.2) -, mimicking the weak coupling between the solid and the fluid subdomains. In this context, our main theoretical results are formulas for the shape derivatives, first of a simple objective function in Proposition 1, second of a general objection function in Proposition 2 and Proposition 3 (the former one giving a volume expression of the shape derivative and the latter one an equivalent surface expression). In a second step, we extend our result to the full three physics problem in Section 4 (our main results are again formulas for the shape derivatives in Propositions Proposition 4 and Proposition 5). Of course, these shapes derivatives are at the basis of our gradient-based optimization algorithm which is used for our numerical simulations.

Another mathematical originality of the present paper lies in the generality of the objective functions considered in our treatment of shape derivatives. Indeed, the most commonly used method for computing a shape derivative is a Eulerian approach, the so-called Lagrangian method of Céa [15] (see also [1, 32, 51]), which has the advantage of being quite simple but relies on the specific knowledge of the formula for the objective function. In other words, if the objective function changes (say from a volume integral to a surface integral), the calculation of the shape derivative has to be performed again from the beginning. Here, following the lead of [40], we rather rely on a Lagrangian approach which allows us to treat very general objective functions, without precise formulas, under a mild assumption on the existence of some partial derivatives. Again, we first explain our results in the simplified setting of Section 3, where we also make a comparison with the more classical Céa's method. Obviously, it is in the more complex framework of the full three-physic problem that our proposed Lagrangian approach is really more efficient than the classical Céa's method. 
From a modeling point of view, the main originality of the present paper is the coupled model of heat propagation, fluid flow and structure deformation it features. There are few previous contributions [59] using this complete model for shape and topology optimization, and they all rely on a completely different method for parametrizing shapes and topologies, namely SIMP [8] or variable density methods. Some simpler variants of this model have been more extensively studied. For example, there are quite many works dealing with convective heat transfer problems (involving coupled fluid and thermal equations, without elasticity) and relying on density methods; see e.g. [37, 22, 62]. Similarly when it comes to optimizing fluid-structure systems (without taking into account thermal effects) one can refer to [60].

As far as the numerical framework is considered, a specificity of our approach - in sharp contrast with the SIMP method - is that we are able to optimize such coupled systems without introducing a relaxed formulation for the description of a mixture of the fluid and solid domain. In the context of the SIMP method (or Brinkman penalization approach for the Navier-Stokes equations [9]), the entire domain is assumed to be filled with a porous material containing a volumic fraction $\eta_{f}$ of fluid and $1-\eta_{f}$ of solid at every point of the domain. Then the state equations posed in the fluid domain $\Omega_{f}$ or in the solid domain $\Omega_{s}$ are replaced with relaxed, homogenized equations to be solved on the whole domain $\Omega=\Omega_{s} \cup \Omega_{f}$ with coefficients depending on the volumic fraction $\eta_{f}$ (if not on a local microstructure, see [2]). In these density approaches, interpolation laws relating the homogenized coefficients to the optimization variable (the material density) must be proposed to penalize adequately intermediate densities and to obtain in practice convergence towards "black and white" designs. One law must be proposed per homogenized coefficient, which makes it delicate to set when considering complex multiphysics problems involving several or many of these coefficients.

On the contrary, our level set approach keeps distinct fluid and solid domains, $\Omega_{f}$ and $\Omega_{s}$, and makes the fluid-solid interface $\Gamma$ the main optimization variable. Keeping track of a clear and neat interface avoids various problems such as mass conservation, load transmission or boundary layers description. Therefore, we propose yet another ingredient which is to mesh exactly the interface at each iteration of the optimization process. In order to still be able to change the topology, we extend the (level set based) mesh evolution algorithm of $[3,4]$ to the present multiphysics setting. In numerical practice, the fluid solid interface $\Gamma$ is evolved with a level set method and remeshed at every iteration for the finite element analysis. This is particularly well suited for our fluid-solid applications, as no alteration of the physical equations is required in contrast with density methods or fixed mesh level set methods, requiring ersatz materials. Another advantage is that it allows the user to use its own favorite finite element solver for each physics, without any need to blend them intrusively.

Shape optimization in fluid mechanics is a very classical problem (see the monographs [34, 46, 39, 47]) and it is known that keeping a clear definition of the boundary is a crucial ingredient for efficiency in many cases. In the context of topology optimization for fluid flows let us quote the recent works [21, 25].

The present article is organized as follows. Section 2 gives a precise account of our weakly coupled model of heat propagation, fluid flow and structure deformation. Section 3 is concerned with our simplified elliptic model, for which we give a full account of the proofs of shape differentiation. Section 4 extends these results to the full multiphysics model (the proofs being postponed to Appendix A). In Section 5 are described our representation of shapes and how they are remeshed after each level set evolution. One advantage of the level set framework is that the topology of the shapes or interfaces can change during the optimization process. Eventually, numerical examples are considered in Section 6. We perform several optimizations using a simple gradient based algorithm, relying on our knowledge of the shape derivatives. Constraints, like volume constraint for one of the fluid or solid phases, are enforced in a classical Lagrangian algorithm. Our first test case is a fluid-structure interaction problem, without taking into account thermal effects. It is inspired from a similar example in [60]. Our second test case is a convective heat transfer problem where the elastic deformation of the solid phase is neglected. It was already studied in [38] with a different approach (based on a variable density relaxation of the problem). Our third and last test case comprises the three physics and is new, to the best of our knowledge. All three examples work nicely in the sense that the objective function is indeed minimized and that the obtained optimal shapes are quite different from the initial ones. These results are preliminary since we have not tried to improve our optimization algorithm (in order to reduce the computational cost) nor did we perform a 3-d implementation. This will be the topic of future work, featuring more industrial test cases and including comparisons with other models, for example a fully coupled fluid-structure interaction system (not merely weakly coupled). 


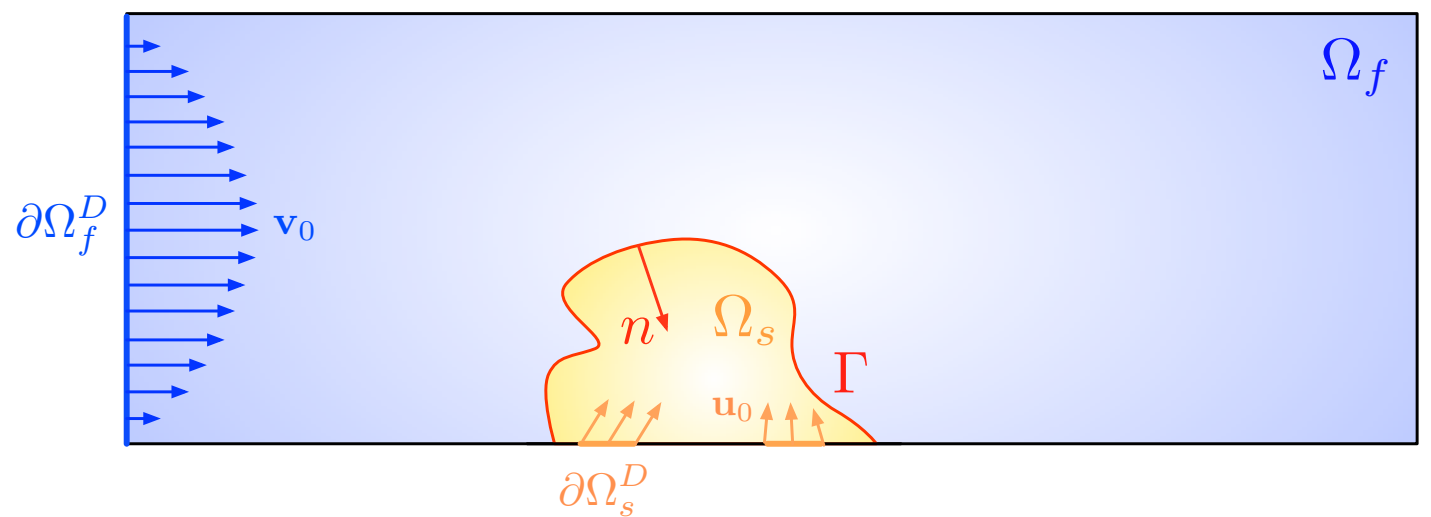

FIGURE 1. Setting of the considered three-physic problem (not all the regions of $\partial \Omega$ featured in (2.1) (2.2) and (2.3) are represented, see the numerical Section 6 for more complete settings).

\section{Setting of the three-Physic PROBlem}

Let $\Omega$ be a fixed, open bounded domain in $\mathbb{R}^{d}$ ( $d=2$ or 3 in applications), arising as the disjoint reunion of a 'fluid' phase $\Omega_{f}$ and a 'solid' phase $\Omega_{s}=\Omega \backslash \overline{\Omega_{f}}$ (see Figure 1), separated by an interface $\Gamma:=\partial \Omega_{f} \cap \partial \Omega_{s}$ which we aim to optimize. Throughout this article, the normal vector $\boldsymbol{n}$ to $\Gamma$ is pointing outward the fluid domain $\Omega_{f}$.

The mechanical behavior of $\Omega$ is governed by three coupled models which we now describe in a formal way, referring to Section 4 for a precise mathematical setting:

- The motion of the fluid inside $\Omega_{f}$;

- The diffusion of heat inside the whole domain $\Omega$;

- The deformation of the solid region $\Omega_{s}$ as a result of the stress exerted by the fluid part and of the dilation entailed by thermoelastic effects.

The fluid domain $\Omega_{f}$ is filled with a fluid characterized by its viscosity $\nu$ and density $\rho$; its velocity $\boldsymbol{v}$ and pressure $p$ satisfy the incompressible steady-state Navier-Stokes equations:

$$
\left\{\begin{aligned}
-\operatorname{div}\left(\sigma_{f}(\boldsymbol{v}, p)\right)+\rho \nabla \boldsymbol{v} v & =\boldsymbol{f}_{f} & & \text { in } \Omega_{f} \\
\operatorname{div}(\boldsymbol{v}) & =0 & & \text { in } \Omega_{f} \\
\boldsymbol{v} & =\boldsymbol{v}_{0} & & \text { on } \partial \Omega_{f}^{D} \\
\sigma_{f}(\boldsymbol{v}, p) \boldsymbol{n} & =0 & & \text { on } \partial \Omega_{f}^{N} \\
\boldsymbol{v} & =0 & & \text { on } \Gamma,
\end{aligned}\right.
$$

where $\nabla \boldsymbol{v}$ is the Jacobian matrix $(\nabla \boldsymbol{v})_{i j}=\partial_{j} \boldsymbol{v}_{i}, i, j=1, \ldots, d$ (note that the nonlinear term $\nabla \boldsymbol{v} \boldsymbol{v}$ can also be written in the more usual way $\boldsymbol{v} \cdot \nabla \boldsymbol{v})$. The fluid stress tensor $\sigma_{f}(\boldsymbol{v}, p)$ is given by

$$
\sigma_{f}(\boldsymbol{v}, p)=2 \nu e(\boldsymbol{v})-p I \text { with } e(\boldsymbol{v})=\left(\nabla \boldsymbol{v}+\nabla \boldsymbol{v}^{T}\right) / 2,
$$

where $I$ is the $d \times d$ identity matrix. In (2.1), $\boldsymbol{f}_{f}$ is an applied body force; the boundary of the fluid phase is the disjoint reunion $\partial \Omega_{f}=\partial \Omega_{f}^{D} \cup \partial \Omega_{f}^{N} \cup \Gamma$ of a Dirichlet (or inlet) part $\partial \Omega_{f}^{D}$ where the flow enters with a given velocity $\boldsymbol{v}=\boldsymbol{v}_{0}$, a Neumann (or outlet) part $\partial \Omega_{f}^{N}$ where zero normal stress is observed, and the interface $\Gamma$ with the solid domain $\Omega_{s}$. At this stage it is assumed that the deformation of the solid domain is sufficiently small so that no slip boundary conditions hold on $\Gamma: \boldsymbol{v}=0$; see Remark 1 below about this point. Therefore, the variables $(\boldsymbol{v}, p)$ depend solely on the geometry of the fluid domain $\Omega_{f}$.

The fluid velocity $\boldsymbol{v}$ determines the physical behavior of the temperature $T$ in the whole domain $\Omega$, as a result of advection and diffusion effects inside the fluid domain $\Omega_{f}$, and of pure diffusion inside the solid domain $\Omega_{s}$. Denoting by $k_{f}$ and $k_{s}$ the thermal conductivity inside $\Omega_{f}$ and $\Omega_{s}$ respectively, and by $c_{p}$ the 
thermal capacity of the fluid, the temperature field $T$ is determined by the steady-state convection-diffusion equations:

$$
\left\{\begin{array}{rlrl}
-\operatorname{div}\left(k_{f} \nabla T_{f}\right)+\rho \boldsymbol{v} \cdot \nabla T_{f} & =Q_{f} & & \text { in } \Omega_{f} \\
-\operatorname{div}\left(k_{s} \nabla T_{s}\right) & =Q_{s} & & \text { in } \Omega_{s} \\
T & =T_{0} & & \text { on } \partial \Omega_{T}^{D} \\
-k_{f} \frac{\partial T_{f}}{\partial n} & =h_{f} & & \text { on } \partial \Omega_{T}^{N} \cap \partial \Omega_{f} \\
-k_{s} \frac{\partial T_{s}}{\partial n} & =h_{s} & & \text { on } \partial \Omega_{T}^{N} \cap \partial \Omega_{s} \\
T_{f} & =T_{s} & & \text { on } \Gamma \\
-k_{f} \frac{\partial T_{f}}{\partial n} & =-k_{s} \frac{\partial T_{s}}{\partial n} & \text { on } \Gamma,
\end{array}\right.
$$

where we use the subscripts ${ }_{f}$ and ${ }_{s}$ for the restrictions $T_{f}$ and $T_{s}$ of $T$ to $\Omega_{f}$ and $\Omega_{s}$ respectively. In (2.2), $Q_{f}$ and $Q_{s}$ are volumic sources inside $\Omega_{f}$ and $\Omega_{s}$; the boundary $\partial \Omega=\partial \Omega_{T}^{N} \cup \partial \Omega_{T}^{D}$ is split into a Dirichlet part, where a temperature $T_{0}$ is imposed and a Neumann part where a given incoming heat flux is observed, which we denote by $h_{f}$ or $h_{s}$ depending on whether it is applied on $\partial \Omega_{T}^{N} \cap \partial \Omega_{f}$ or $\partial \Omega_{T}^{N} \cap \partial \Omega_{s}$. The temperature $T$ as well as the heat flux are continuous across the interface $\Gamma$ between $\Omega_{f}$ and $\Omega_{s}$.

Finally, the fluid variables $(\boldsymbol{v}, p)$ and the temperature $T$ together determine the displacement $\boldsymbol{u}$ of the solid domain $\Omega_{s}$, which is assumed to consist of an isotropic thermoelastic material with Lamé coefficients $\lambda, \mu$, thermal expansion parameter $\alpha$ and temperature at rest $T_{\text {ref }}$. This variable $\boldsymbol{u}$ is characterized by the equations of linear thermoelasticity:

$$
\left\{\begin{aligned}
-\operatorname{div}\left(\sigma_{s}\left(\boldsymbol{u}, T_{s}\right)\right) & =\boldsymbol{f}_{s} & & \text { in } \Omega_{s} \\
\boldsymbol{u} & =\boldsymbol{u}_{0} & & \text { on } \partial \Omega_{s}^{D} \\
\sigma_{s}\left(\boldsymbol{u}, T_{s}\right) \cdot \boldsymbol{n} & =\boldsymbol{g} & & \text { on } \partial \Omega_{s}^{N} \\
\sigma_{s}\left(\boldsymbol{u}, T_{s}\right) \cdot \boldsymbol{n} & =\sigma_{f}(\boldsymbol{v}, p) \cdot \boldsymbol{n} & & \text { on } \Gamma .
\end{aligned}\right.
$$

where the solid stress tensor is given by

$$
\sigma_{s}\left(\boldsymbol{u}, T_{s}\right)=A e(\boldsymbol{u})-\alpha\left(T_{s}-T_{\mathrm{ref}}\right) I \text { with } A e(\boldsymbol{u})=2 \mu e(\boldsymbol{u})+\lambda \operatorname{Tr}(e(\boldsymbol{u})) I,
$$

and $\boldsymbol{f}_{s}$ is an applied body force. In (2.3), the boundary $\partial \Omega_{s}$ is split into respectively a Dirichlet part $\partial \Omega_{s}^{D}$ where a displacement $\boldsymbol{u}=\boldsymbol{u}_{0}$ is prescribed, a Neumann part $\partial \Omega_{s}^{N}$ where a stress $\boldsymbol{g}$ is imposed, and the interface $\Gamma$ with the fluid domain. This latter boundary is submitted to the friction force imposed by the fluid, which translates into the equality $\sigma_{f}(\boldsymbol{v}, p) \cdot \boldsymbol{n}=\sigma_{s}\left(\boldsymbol{u}, T_{s}\right) \cdot \boldsymbol{n}$ between the normal fluid and solid stresses.

Remark 1. The above model is a simplified version of a genuine fluid-solid-thermic coupling between the phases $\Omega_{f}$ and $\Omega_{s}$. As a matter of fact, a more accurate description of fluid-structure interaction would feature a vanishing fluid velocity $\boldsymbol{v}$ on the deformed interface $(\operatorname{Id}+\boldsymbol{u})(\Gamma)$, namely:

$$
\boldsymbol{v}(x+\boldsymbol{u}(x))=0, x \in \Gamma,
$$

see e.g. [48], or [60] in an optimization context. Likewise, the equality between normal stresses $\sigma_{s}\left(\boldsymbol{u}, T_{s}\right) \boldsymbol{n}=$ $\sigma_{f}(\boldsymbol{v}, p) \boldsymbol{n}$ should hold on the deformed interface. In the present article, we assume that the displacement $\boldsymbol{u}$ of the solid phase is small enough so that the influence of the interface deformation on the physical behavior of the fluid can be neglected. Notice that, thanks to these simplifications, the system (2.1) to (2.3) is only weakly coupled: its resolution is achieved by solving first the fluid system (2.1), then using the resulting fluid velocity $\boldsymbol{v}$ in the heat transfer equation (2.2), then using the fluid stress $\sigma_{f}(\boldsymbol{v}, p)$ and the temperature $T_{s}$ to solve (2.3).

\section{A Simplified SCALAR FLUid STRUCTURE INTERACTION PROBlem}

One of the salient features of the system (2.1) to (2.3) is the equality of normal stresses $\sigma_{s}\left(\boldsymbol{u}, T_{s}\right) \boldsymbol{n}=$ $\sigma_{f}(\boldsymbol{v}, p) \boldsymbol{n}$ imposed on the optimized interface $\Gamma$. The calculation of shape derivatives in this context is not a completely standard issue to the best of our knowledge, and the result has an interesting structure: the 
boundary conditions for the adjoint systems are different from those appearing in the state equations (see the expressions (4.9) and (4.11)). To illustrate this fact, we first consider a simplified scalar problem which gathers with lighter notations all the essential mathematical points.

\subsection{Presentation of the simplified problem.}

We consider the following setting: the fluid variable $\boldsymbol{v}$ is replaced by a scalar variable $u_{f}$ solving the Poisson equation (3.1) in $\Omega_{f}$ with homogeneous Dirichlet boundary conditions on the interface $\Gamma$. The elastic variable $\boldsymbol{u}$ is replaced by the solution $u_{s}$ to another Poisson equation (3.2) posed in the complement $\Omega_{s}$. Sources $f_{f} \in H^{1}\left(\Omega_{f}\right), f_{s} \in H^{1}\left(\Omega_{s}\right)$, with the dimension of a force, are applied in both cases. Note that such $H^{1}$ regularity is needed when computing shape derivatives in the sequel (e.g. in (3.36)), although weaker regularity would be enough for state equations to be well-posed. For the sake of physical units $\left(u_{f}\right.$ and $u_{s}$ have respectively the dimension of a velocity and of a displacement), the Laplace operators involved in (3.1) and (3.2) are scaled with constants $\nu$ and $\mu$ that assume respectively the role of the viscosity in the fluid domain, and of a Lamé coefficient in the solid domain. The two systems for $u_{f}$ and $u_{s}$ are weakly coupled: the equation (3.1) for $u_{f}$ does not depend on $u_{s}$ and in the system (3.2) for $u_{s}$, equality of normal fluxes is imposed at the interface $\Gamma$, as the exact counterpart in the scalar setting of the continuity of normal stresses $\sigma_{s}\left(\boldsymbol{u}, T_{s}\right) \boldsymbol{n}=\sigma_{f}(\boldsymbol{v}, p) \boldsymbol{n}$. For simplicity, homogeneous Dirichlet boundary conditions are imposed on $\partial \Omega$ in both systems for $u_{f}$ and $u_{s}$ :

$$
\begin{gathered}
\left\{\begin{array}{rrr}
-\nu \Delta u_{f}=f_{f} & \text { in } \Omega_{f} \\
u_{f}=0 & \text { on } \Gamma \\
u_{f}=0 & \text { on } \partial \Omega_{f} \backslash \Gamma,
\end{array}\right. \\
\left\{\begin{array}{rr}
-\mu \Delta u_{s}=f_{s} & \text { in } \Omega_{s} \\
\mu \frac{\partial u_{s}}{\partial n}=\nu \frac{\partial u_{f}}{\partial n} & \text { on } \Gamma \\
u_{s}=0 & \text { on } \partial \Omega_{s} \backslash \Gamma,
\end{array}\right.
\end{gathered}
$$

where we recall our convention that the normal vector $\boldsymbol{n}$ in (3.2) is pointing outward $\Omega_{f}$ (the Neumann boundary condition is equally valid if $\boldsymbol{n}$ is pointing outward $\Omega_{s}$ ). Our aim is to solve the minimization problem

$$
\min _{\Gamma} J\left(\Gamma, u_{f}(\Gamma), u_{s}(\Gamma)\right)
$$

where $J$ is a given cost function upon which we shall impose adequate regularity conditions in due time. Usually, (3.3) is enriched with constraints, e.g. on the volume or the perimeter of one of the phases $\Omega_{f}, \Omega_{s}$; we leave aside the consideration of such classical shape functionals for the mathematical analysis.

In the sequel, the dependence of the state variables $u_{f}$ and $u_{s}$ with respect to $\Gamma$ shall be made explicit - using the notations $u_{s}(\Gamma)$ and $u_{f}(\Gamma)$ as in (3.3) - only when it comes to distinguishing these from generic functions defined on $\Omega_{f}$ and $\Omega_{s}$ (e.g. as in. (3.26) below). In order to discuss the precise mathematical setting for (3.1) (3.2), we introduce the following spaces of functions on the subdomains $\Omega_{s}$ and $\Omega_{f}$ :

$$
\begin{aligned}
V_{s}(\Gamma) & =\left\{v_{s} \in H^{1}\left(\Omega_{s}\right) \mid v_{s}=0 \text { on } \partial \Omega_{s} \backslash \Gamma\right\}, \\
V_{f}(\Gamma) & =\left\{v_{f} \in H^{1}\left(\Omega_{f}\right) \mid v_{f}=0 \text { on } \partial \Omega_{f} \backslash \Gamma\right\}, \\
V_{f, 0}(\Gamma) & =\left\{v_{f} \in H^{1}\left(\Omega_{f}\right) \mid v_{f}=0 \text { on } \partial \Omega_{f}\right\} .
\end{aligned}
$$

We also consider the subspace $H_{00}^{1 / 2}(\Gamma)$ of $H^{1 / 2}(\Gamma)$ composed of restrictions to $\Gamma$ of functions in $V_{f}(\Gamma)$,

$$
H_{00}^{1 / 2}(\Gamma)=\left\{\left.v\right|_{\Gamma} \mid v \in V_{f}(\Gamma)\right\},
$$

and its dual space $H_{00}^{-1 / 2}(\Gamma)$. Roughly speaking, any element $v \in H_{00}^{1 / 2}(\Gamma)$ has an extension $v_{f}$ to $\Omega_{f}$ vanishing on $\partial \Omega_{f} \backslash \Gamma[36]$. 
In this framework, the state variables $u_{s}$ and $u_{f}$ in (3.1) and (3.2) are the unique solutions to the following variational problems:

$$
\text { Find } u_{f} \in V_{f, 0}(\Gamma) \text { such that } \forall v_{f} \in V_{f, 0}(\Gamma), \int_{\Omega_{f}} \nu \nabla u_{f} \cdot \nabla v_{f} \mathrm{~d} x=\int_{\Omega_{f}} f_{f} v_{f} \mathrm{~d} x
$$

$$
\text { Find } u_{s} \in V_{s}(\Gamma) \text { such that } \forall v_{s} \in V_{s}(\Gamma), \int_{\Omega_{s}} \mu \nabla u_{s} \cdot \nabla v_{s} \mathrm{~d} x=\int_{\Omega_{s}} f_{s} v_{s} \mathrm{~d} x-\int_{\Gamma} \nu \frac{\partial u_{f}}{\partial n} v_{s} \mathrm{~d} s,
$$

where the minus sign in the last term of the right-hand side of (3.9) is due to our convention whereby $\boldsymbol{n}$ is pointing outward $\Omega_{f}$.

A comment is in order about the meaning of (3.9). In general, the normal derivative $\frac{\partial v}{\partial n}$ on the interface $\Gamma$ of an arbitrary function $v \in V_{f, 0}(\Gamma)$ is not defined, as there is no trace theorem for the gradient of functions in $H^{1}\left(\Omega_{f}\right)$. However, $u_{f}$ is not a mere function of $H^{1}\left(\Omega_{f}\right)$ : since it satisfies (3.8), one also has $\Delta u_{f}=f_{f} \in L^{2}\left(\Omega_{f}\right)$. Therefore, the last term of $(3.9)$ is defined, for any $v \in H_{00}^{1 / 2}(\Gamma)$, by

$$
-\int_{\Gamma} \nu \frac{\partial u_{f}}{\partial n} v \mathrm{~d} s:=-\int_{\Omega_{f}}\left(\nu \Delta u_{f} \tilde{v}+\nu \nabla u_{f} \cdot \nabla \tilde{v}\right) \mathrm{d} x=\int_{\Omega_{f}}\left(f_{f} \tilde{v}-\nu \nabla u_{f} \cdot \nabla \tilde{v}\right) \mathrm{d} x,
$$

where $\tilde{v} \in V_{f}(\Gamma)$ is any extension satisfying $\tilde{v}=v$ on $\Gamma$. Note that, for smooth $u_{f}$, (3.10) is just Green's formula. Since from (3.8), the right-hand side of (3.10) does not depend on the choice of such extension $\tilde{v}$, this identity actually defines $\frac{\partial u_{f}}{\partial n}$ as an element of the dual $H_{00}^{-1 / 2}(\Gamma)$; see e.g. [36].

The variational formulation (3.9) associated to (3.2) thus rewrites:

$$
\begin{aligned}
& \text { Find } u_{s} \in V_{s}(\Gamma) \text { such that } \forall v_{s} \in V_{s}(\Gamma) \text {, } \\
& \qquad \int_{\Omega_{s}} \mu \nabla u_{s} \cdot \nabla v_{s} \mathrm{~d} x=\int_{\Omega_{s}} f_{s} v_{s} \mathrm{~d} x+\int_{\Omega_{f}} f_{f} \tilde{v}_{s} \mathrm{~d} x-\int_{\Omega_{f}} \nu \nabla u_{f} \cdot \nabla \tilde{v}_{s} \mathrm{~d} x
\end{aligned}
$$

where $\tilde{v}_{s} \in V_{f}(\Gamma)$ is any extension of $v_{s}$ to $\Omega_{f}$ such that $\tilde{v}_{s}=v_{s}$ on $\Gamma$.

Remark 2. When $\Gamma \cap \partial \Omega=\emptyset$, which happens if for instance $\Omega_{s} \Subset \Omega, H_{00}^{1 / 2}(\Gamma)$ and $H_{00}^{-1 / 2}(\Gamma)$ coincide with the more usual fractional Sobolev spaces $H^{1 / 2}(\Gamma)$ and $H^{-1 / 2}(\Gamma)$ respectively; see [36, 54] about these technicalities.

\subsection{Hadamard's boundary variation method for shape optimization.}

The numerical resolution of the shape optimization problem (3.3) requires a notion of differentiation with respect to the domain. In this article, we rely on Hadamard's method for boundary variations [32, 1, 51], which considers variations of the interface $\Gamma$ of the form (see Figure 2)

$$
\Gamma_{\boldsymbol{\theta}}=(I+\boldsymbol{\theta}) \Gamma \text {, where } \boldsymbol{\theta} \in W_{0}^{1, \infty}\left(\Omega, \mathbb{R}^{d}\right),\|\boldsymbol{\theta}\|_{W^{1, \infty}\left(\mathbb{R}^{d}, \mathbb{R}^{d}\right)}<1 .
$$

Recall that, in the above circumstances, $(I+\boldsymbol{\theta})$ is a Lipschitz diffeomorphism from $\mathbb{R}^{d}$ into itself [27]. Since in our context only the interface $\Gamma$ is subject to optimization, and not the boundary $\partial \Omega$ of the total domain, the admissible space for deformations $\boldsymbol{\theta}$ is:

$$
W_{0}^{1, \infty}\left(\Omega, \mathbb{R}^{d}\right)=\left\{\boldsymbol{\theta} \in L^{\infty}\left(\Omega, \mathbb{R}^{d}\right) \mid \nabla \boldsymbol{\theta} \in L^{\infty}\left(\Omega, \mathbb{R}^{d} \times \mathbb{R}^{d}\right) \text { and } \boldsymbol{\theta}=0 \text { on } \partial \Omega\right\} .
$$

Remark 3. The condition $\boldsymbol{\theta}=0$ on $\partial \Omega$ implies that junction points corresponding to the intersection $\partial \Omega \cap \Gamma$ are fixed. The analysis to follow can of course be extended to more general shape variations $(e . g$. only $\boldsymbol{\theta} \cdot \boldsymbol{n}=0$ on $\partial \Omega$ or even $\boldsymbol{\theta} \in W^{1, \infty}\left(\Omega, \mathbb{R}^{d}\right)$ ), at the expense of including extra terms in shape derivatives accounting for the variations of boundary conditions or for the tangential displacements of the junction points.

The shape derivative of a function $J(\Gamma)$ is defined as the Fréchet derivative $\frac{\mathrm{d} J}{\mathrm{~d} \boldsymbol{\theta}}$ of the underlying mapping $\boldsymbol{\theta} \mapsto J\left(\Gamma_{\boldsymbol{\theta}}\right)$, from $W_{0}^{1, \infty}\left(\Omega, \mathbb{R}^{d}\right)$ into $\mathbb{R}$ at $\boldsymbol{\theta}=0$ :

$$
J\left(\Gamma_{\boldsymbol{\theta}}\right)=J(\Gamma)+\frac{\mathrm{d} J}{\mathrm{~d} \boldsymbol{\theta}}(\boldsymbol{\theta})+o(\boldsymbol{\theta}), \quad \text { where } \frac{|o(\boldsymbol{\theta})|}{\|\boldsymbol{\theta}\|_{W^{1, \infty}\left(\Omega, \mathbb{R}^{d}\right)}} \stackrel{\boldsymbol{\theta} \rightarrow 0}{\longrightarrow} 0,
$$




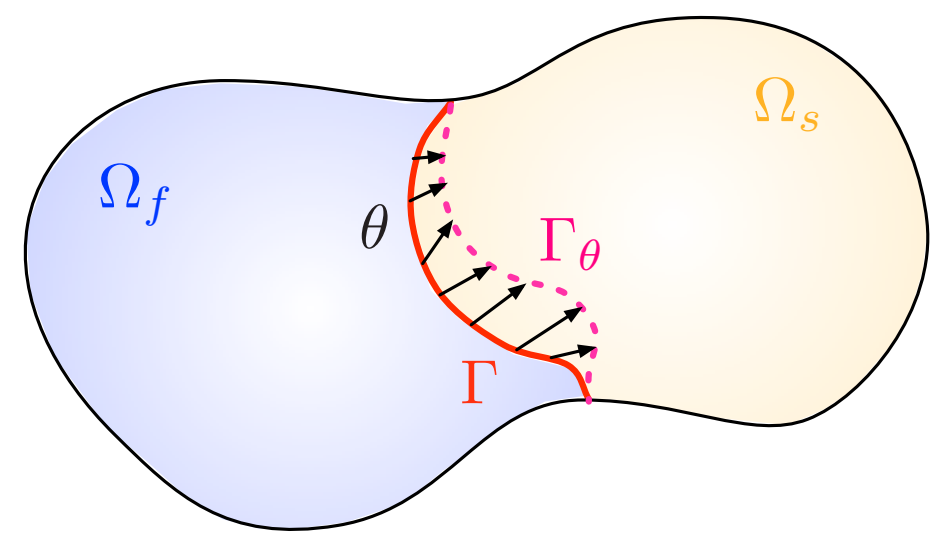

FIGURE 2. Deformation of a partition $\Omega=\Omega_{f} \cup \Omega_{s}$ using Hadamard's method.

where, with a little abuse of notations, we have also denoted by $\boldsymbol{\theta}$ the direction in which $\boldsymbol{\theta} \mapsto J\left(\Gamma_{\boldsymbol{\theta}}\right)$ is differentiated. In (3.14), $\frac{\mathrm{d} J}{\mathrm{~d} \boldsymbol{\theta}}$ appears to be an element of the dual space of $W_{0}^{1, \infty}\left(\Omega, \mathbb{R}^{d}\right)$. If sufficient regularity holds for the shape derivative $\frac{\mathrm{d} J}{\mathrm{~d} \boldsymbol{\theta}}$ to be continuous over some Hilbert space, say $H_{0}^{1}\left(\Omega, \mathbb{R}^{d}\right)$, equipped with an arbitrary inner product $a(\cdot, \cdot)$, then a descent direction $\boldsymbol{\theta}$ is obtained by identifying the linear form $\frac{\mathrm{d} J}{\mathrm{~d} \boldsymbol{\theta}}$ with a gradient, using the Riesz representation theorem [14, 23]:

$$
\text { Find } \boldsymbol{\theta} \in H_{0}^{1}\left(\Omega, \mathbb{R}^{d}\right) \text {, such that } \forall \boldsymbol{\theta}^{\prime} \in H_{0}^{1}\left(\Omega, \mathbb{R}^{d}\right), a\left(\boldsymbol{\theta}, \boldsymbol{\theta}^{\prime}\right)=-\frac{\mathrm{d} J}{\mathrm{~d} \boldsymbol{\theta}}\left(\boldsymbol{\theta}^{\prime}\right) .
$$

The fact that $\boldsymbol{\theta}$ is indeed a descent direction results from the fact that $\frac{\mathrm{d} J}{\mathrm{~d} \boldsymbol{\theta}}(\boldsymbol{\theta})=-a(\boldsymbol{\theta}, \boldsymbol{\theta}) \leq 0$ together with (3.14). In numerical practice, $a$ is often defined as

$$
\forall \boldsymbol{\theta}, \boldsymbol{\theta}^{\prime} \in H_{0}^{1}\left(\Omega, \mathbb{R}^{d}\right), a\left(\boldsymbol{\theta}, \boldsymbol{\theta}^{\prime}\right)=\int_{\Omega}\left(\gamma^{2} \nabla \boldsymbol{\theta}: \nabla \boldsymbol{\theta}^{\prime}+\boldsymbol{\theta} \cdot \boldsymbol{\theta}^{\prime}\right) \mathrm{d} x
$$

where $\gamma$ is a tunable regularizing parameter selected in function of the mesh resolution.

\subsection{Calculating the shape derivative of a particular objective functional and its adjoint system with Céa's method.}

We first calculate the shape derivative of a quite general objective function $J$ of the interface $\Gamma$ - and we exhibit the corresponding adjoint systems (including their boundary conditions) - by using the classical Céa's Lagrangian method [15]. In this subsection (and only here), we restrict ourselves to objective functionals $J$ of the form:

$$
J\left(\Gamma, u_{s}(\Gamma), u_{f}(\Gamma)\right)=\int_{\Omega_{f}} j_{f}\left(u_{f}(\Gamma)\right) \mathrm{d} x+\int_{\Omega_{s}} j_{s}\left(u_{s}(\Gamma)\right) \mathrm{d} x
$$

for two $C^{2}$ functions $j_{s}, j_{f}: \mathbb{R} \rightarrow \mathbb{R}$ with bounded second-order derivatives: $\left\|j_{s}^{\prime \prime}\right\|_{L^{\infty}(\mathbb{R})}<\infty,\left\|j_{f}^{\prime \prime}\right\|_{L^{\infty}(\mathbb{R})}<$ $\infty$.

Let us define the adjoint variables $p_{s} \in V_{s}(\Gamma)$ and $p_{f} \in V_{f}(\Gamma)$ by:

$$
\left\{\begin{aligned}
-\mu \Delta p_{s} & =j_{s}^{\prime}\left(u_{s}\right) \text { in } \Omega_{s} \\
\mu \frac{\partial p_{s}}{\partial n} & =0 \text { on } \Gamma \\
p_{s} & =0 \text { on } \partial \Omega_{s} \backslash \Gamma,
\end{aligned}\right.
$$




$$
\left\{\begin{aligned}
-\nu \Delta p_{f} & =j_{f}^{\prime}\left(u_{f}\right) \text { in } \Omega_{f} \\
p_{f} & =p_{s} \text { on } \Gamma \\
p_{f} & =0 \text { on } \partial \Omega_{f} \backslash \Gamma .
\end{aligned}\right.
$$

Interestingly, the equality between normal derivatives $\nu \frac{\partial u_{f}}{\partial n}=\mu \frac{\partial u_{s}}{\partial n}$ featured in the system (3.2) for the state variables translates into the rather surprising boundary condition $p_{f}=p_{s}$ on $\Gamma$ in (3.19) for the adjoint variables $p_{f}$. This boundary condition can be obtained in at least two ways. In the (formal) proof of Proposition 1 below, the adjoint boundary condition arises from the usual definition of the Lagrangian in Céa's method. In Section 3.4.4, we provide an equivalent mixed formulation for the state equations (3.1) and (3.2) which features different spaces for the 'primal' state variables and the 'adjoint' test functions. This difference of functional spaces is at the root of this change of boundary conditions at the interface.

Proposition 1. The functional $\boldsymbol{\theta} \mapsto J\left(\Gamma_{\boldsymbol{\theta}}, u_{s}\left(\Gamma_{\boldsymbol{\theta}}\right), u_{f}\left(\Gamma_{\boldsymbol{\theta}}\right)\right)$, from $W_{0}^{1, \infty}\left(\Omega, \mathbb{R}^{d}\right)$ into $\mathbb{R}$, as defined in (3.17), is differentiable at $\boldsymbol{\theta}=0$ and, under $H^{2}$ regularity of the variables $u_{f}, u_{s}, p_{f}, p_{s}$, the shape derivative reads:

$$
\begin{aligned}
& \frac{\mathrm{d}}{\mathrm{d} \boldsymbol{\theta}}\left[J\left(\Gamma_{\boldsymbol{\theta}}, u_{f}\left(\Gamma_{\boldsymbol{\theta}}\right), u_{s}\left(\Gamma_{\boldsymbol{\theta}}\right)\right)\right](\boldsymbol{\theta})= \\
& \int_{\Gamma}\left[j_{f}\left(u_{f}\right)-j_{s}\left(u_{s}\right)+f_{f} p_{f}-f_{s} p_{s}+\mu \nabla u_{s} \cdot \nabla p_{s}+\nu \frac{\partial u_{f}}{\partial n} \frac{\partial p_{f}}{\partial n}\right](\boldsymbol{\theta} \cdot \boldsymbol{n}) \mathrm{d} s .
\end{aligned}
$$

Remark 4. Formula (3.20) is equivalent to the following one, which shall be recovered by a different method in Section 3.4,

$$
\begin{aligned}
& \frac{\mathrm{d}}{\mathrm{d} \boldsymbol{\theta}}\left[J\left(\Gamma_{\boldsymbol{\theta}}, u_{f}\left(\Gamma_{\boldsymbol{\theta}}\right), u_{s}\left(\Gamma_{\boldsymbol{\theta}}\right)\right)\right](\boldsymbol{\theta})= \\
& \quad \int_{\Gamma}\left[j_{f}\left(u_{f}\right)-j_{s}\left(u_{s}\right)+f_{f} p_{f}-f_{s} p_{s}-\nu \nabla u_{f} \cdot \nabla p_{f}+\mu \nabla u_{s} \cdot \nabla p_{s}+2 \nu \frac{\partial u_{f}}{\partial n} \frac{\partial p_{f}}{\partial n}\right](\boldsymbol{\theta} \cdot \boldsymbol{n}) \mathrm{d} s
\end{aligned}
$$

because the boundary condition $u_{f}=0$ on $\Gamma$ implies that $\nu \frac{\partial u_{f}}{\partial n} \frac{\partial p_{f}}{\partial n}=\nu \nabla u_{f} \cdot \nabla p_{f}$.

Proof. We introduce the following Lagrangian

$$
\begin{aligned}
\mathcal{L}\left(\Gamma, \hat{u}_{f}, \hat{u}_{s}, \hat{p}_{f}, \hat{p}_{s}, \hat{\lambda}\right)=\int_{\Omega_{f}} j_{f}\left(\hat{u}_{f}\right) \mathrm{d} x+\int_{\Omega_{s}} j_{s}\left(\hat{u}_{s}\right) \mathrm{d} x \\
\quad-\int_{\Omega_{f}}\left(-\nu \Delta \hat{u}_{f}-f_{f}\right) \hat{p}_{f} \mathrm{~d} x-\int_{\Omega_{s}}\left(\mu \nabla \hat{u}_{s} \cdot \nabla \hat{p}_{s}-f_{s} \hat{p}_{s}\right) \mathrm{d} x-\int_{\Gamma} \nu \frac{\partial \hat{u}_{f}}{\partial n} \hat{p}_{s} \mathrm{~d} s-\int_{\Gamma} \hat{\lambda} \hat{u}_{f} \mathrm{~d} s
\end{aligned}
$$

where the above 'hat' functions all belong to the Sobolev space $H_{0}^{1}(\Omega)$ which is independent of the position of the interface $\Gamma$. Following the methodology described in $[1,15,43]$, Lagrange multipliers $\hat{p}_{f}, \hat{p}_{s}, \hat{\lambda}$ are introduced in (3.22) to enforce the state equations (3.1) and (3.2) and the Dirichlet boundary condition $u_{f}=0$ on the moving interface $\Gamma$. The main idea of Céa's method consists in finding the equations for the values $p_{f}, p_{s}$ of the adjoint states $\hat{p}_{f}$ and $\hat{p}_{s}$ and the value $\lambda$ of the Lagrange multiplier $\hat{\lambda}$ by requiring that the partial derivatives $\frac{\partial \mathcal{L}}{\partial \hat{u}_{f}}, \frac{\partial \mathcal{L}}{\partial \hat{u}_{s}}$ at $\hat{u}_{f}=u_{f}, \hat{u}_{s}=u_{s}$ vanish. The latter partial derivatives read, for arbitrary $v_{f}, v_{s} \in H_{0}^{1}(\Omega)$ :

$$
\begin{gathered}
\frac{\partial \mathcal{L}}{\partial \hat{u}_{f}}\left(v_{f}\right)=\int_{\Omega_{f}} j_{f}^{\prime}\left(u_{f}\right) v_{f} \mathrm{~d} x+\int_{\Omega_{f}} \nu \Delta v_{f} p_{f} \mathrm{~d} x-\int_{\Gamma} \nu \frac{\partial v_{f}}{\partial n} p_{s} \mathrm{~d} s-\int_{\Gamma} \lambda v_{f} \mathrm{~d} s \\
=\int_{\Omega_{f}}\left(j_{f}^{\prime}\left(u_{f}\right)+\nu \Delta p_{f}\right) v_{f} \mathrm{~d} x+\int_{\Gamma} \nu\left(\frac{\partial v_{f}}{\partial n} p_{f}-\frac{\partial p_{f}}{\partial n} v_{f}\right) \mathrm{d} s-\int_{\Gamma} \nu \frac{\partial v_{f}}{\partial n} p_{s} \mathrm{~d} s-\int_{\Gamma} \lambda v_{f} \mathrm{~d} s \\
\frac{\partial \mathcal{L}}{\partial \hat{u}_{s}}\left(v_{s}\right)=\int_{\Omega_{s}} j_{s}^{\prime}\left(u_{s}\right) v_{s} \mathrm{~d} x-\int_{\Omega_{s}} \mu \nabla v_{s} \cdot \nabla p_{s} \mathrm{~d} x
\end{gathered}
$$

Now requiring $\frac{\partial \mathcal{L}}{\partial \hat{u}_{s}}\left(v_{s}\right)$ to vanish for any $v_{s} \in H_{0}^{1}(\Omega)$ yields the adjoint equation (and the attached boundary conditions) (3.18) for $p_{s}$. 
Likewise, requiring $\frac{\partial \mathcal{L}}{\partial \hat{u}_{f}}\left(v_{f}\right)$ to vanish for any $v_{f} \in H_{0}^{1}(\Omega)$ should lead to the other adjoint system (3.19) but the derivation is a bit more subtle. First, choosing arbitrary $v_{f}$ with compact support in $\Omega_{f}$ in (3.23) yields:

$$
-\nu \Delta p_{f}=j_{f}^{\prime}\left(u_{f}\right)
$$

Second, choosing smooth $v_{f}$ such that $v_{f}=0$ on $\Gamma$ and that $v_{f}$ has an arbitrary trace $\frac{\partial v_{f}}{\partial n}$ yields the Dirichlet interface condition $p_{f}=p_{s}$ on $\Gamma$. Thus, the adjoint system (3.19) for $p_{f}$ is completely recovered. Finally, choosing $v_{f}$ in (3.23) with arbitrary trace on $\Gamma$ leads to the optimal value of the Lagrange multiplier $\lambda=-\nu \frac{\partial p_{f}}{\partial n}$ on $\Gamma$.

Assuming that the solutions $u_{s}$ and $u_{f}$ to (3.1) and (3.2) are differentiable with respect to $\Gamma$ (which is where Céa's method is only formal), and using that the partial derivatives of $\mathcal{L}$ with respect to $\hat{u}_{f}$ and $\hat{u}_{s}$ vanish at $\left(\boldsymbol{\theta}, \hat{u}_{f}, \hat{u}_{s}, \hat{p}_{f}, \hat{p}_{s}, \hat{\lambda}\right)=\left(0, u_{f}, u_{s}, p_{f}, p_{s}, \lambda\right)$, a simple use of the chain rule produces:

$$
\begin{aligned}
& \frac{\mathrm{d}}{\mathrm{d} \boldsymbol{\theta}}\left[J\left(\Gamma_{\boldsymbol{\theta}}, u_{f}\left(\Gamma_{\boldsymbol{\theta}}\right), u_{s}\left(\Gamma_{\boldsymbol{\theta}}\right)\right)\right](\boldsymbol{\theta})=\frac{\partial \mathcal{L}}{\partial \boldsymbol{\theta}}(\boldsymbol{\theta}) \\
& \quad=\int_{\Gamma}\left(j_{f}\left(u_{f}\right)-j_{s}\left(u_{s}\right)+\mu \nabla u_{s} \cdot \nabla p_{s}-f_{s} p_{s}-\nu \operatorname{div}\left(p_{s} \cdot \nabla u_{f}\right)-\lambda \frac{\partial u_{f}}{\partial n}\right)(\boldsymbol{\theta} \cdot \boldsymbol{n}) \mathrm{d} s \\
& \quad=\int_{\Gamma}\left(j_{f}\left(u_{f}\right)-j_{s}\left(u_{s}\right)+\mu \nabla u_{s} \cdot \nabla p_{s}-f_{s} p_{s}-\nu \nabla p_{s} \cdot \nabla u_{f}+f_{f} p_{s}+\nu \frac{\partial u_{f}}{\partial n} \frac{\partial p_{f}}{\partial n}\right)(\boldsymbol{\theta} \cdot \boldsymbol{n}) \mathrm{d} s,
\end{aligned}
$$

where we have used the technical Lemma 1 below when differentiating $\int_{\Gamma} \nu \frac{\partial u_{f}}{\partial n} p_{s} \mathrm{~d} s$ since the normal vector depends on $\Gamma$. Using that $u_{f}=0$ and $\frac{\partial p_{s}}{\partial n}=0$ on $\Gamma$, it follows that $\nabla u_{f} \cdot \nabla p_{s}=0$ on $\Gamma$, and since $p_{f}=p_{s}$ on $\Gamma$, we retrieve expression (3.21).

The following result was used in the above proof for differentiating a boundary integral involving the normal with respect to the shape; see $[19,6,32,51]$ :

Lemma 1. Let $\boldsymbol{f} \in W^{2,1}\left(\Omega, \mathbb{R}^{d}\right)$ and assume $\Gamma$ to be of class $\mathcal{C}^{1}$. Denote $\boldsymbol{n}_{\boldsymbol{\theta}}$ the normal vector on the deformed boundary $(I+\boldsymbol{\theta}) \Gamma$ oriented outward $(I+\boldsymbol{\theta}) \Omega_{f}$. Then, for arbitrary variations $\boldsymbol{\theta} \in \mathcal{C}^{1}\left(\Omega, \mathbb{R}^{d}\right)$ vanishing on $\partial \Omega$,

$$
\frac{\mathrm{d}}{\mathrm{d} \boldsymbol{\theta}}\left[\int_{\Gamma_{\boldsymbol{\theta}}} \boldsymbol{f} \cdot \boldsymbol{n}_{\boldsymbol{\theta}} \mathrm{d} s\right](\boldsymbol{\theta})=\int_{\Gamma} \operatorname{div}(\boldsymbol{f})(\boldsymbol{\theta} \cdot \boldsymbol{n}) \mathrm{d} s .
$$

\subsection{A fully Lagrangian setting for computing shape derivatives of arbitrary objective func- tionals.}

Although very common and widely used in the literature (see e.g. [6, 43]), an issue with Céa's method as exposed in the previous section is that the computation of the shape derivative of an objective function $J$ depends very much on the assumptions made on the nature of $J$. Different objective functionals may lead to different strong forms for the adjoint equations, which imposes to redo the analytical derivation whenever changing the objective function, and to update the numerical implementation accordingly.

In this section, we use a fully Lagrangian setting to derive rigorously the shape derivative of very general objective functionals, in the spirit of the seminal work of Murat and Simon [40]. We first calculate the shape sensitivities of the state variables $u_{f}(\Gamma), u_{s}(\Gamma)$ before calculating the shape derivative of an arbitrary objective functional in volume form. Then, under sufficient regularity assumptions, using the well-known Hadamard structure theorem together with suitable integration by parts yields general shape derivative formulas in the classical form of a boundary integral.

\subsubsection{A modified objective functional and Lagrangian derivative of the state variables.}

The starting remark is that the functional $J$, although appearing naturally in the formulation of the optimization problem (3.3), is not so convenient for the mathematical analysis. Indeed, the domain of definition of $J(\Gamma, \cdot, \cdot)$ is $V_{f, 0}(\Gamma) \times V_{s}(\Gamma)$, a functional space which depends on the first argument $\Gamma$. In order to address this issue, the classical idea is to work in a Lagrangian framework rather than an Eulerian one. Therefore, we 
consider a fixed reference interface $\Gamma$, and we introduce a modified functional $\mathfrak{J}$ obtained by "transporting" $J$ on a fixed space: for any $\left(\boldsymbol{\theta}, \hat{u}_{f}, \hat{u}_{s}\right) \in W_{0}^{1, \infty}\left(\Omega, \mathbb{R}^{d}\right) \times V_{f, 0}(\Gamma) \times V_{s}(\Gamma)$,

$$
\mathfrak{J}\left(\boldsymbol{\theta}, \hat{u}_{f}, \hat{u}_{s}\right):=J\left(\Gamma_{\boldsymbol{\theta}}, \hat{u}_{f} \circ(I+\boldsymbol{\theta})^{-1}, \hat{u}_{s} \circ(I+\boldsymbol{\theta})^{-1}\right) .
$$

Conversely, this allows to rewrite the objective functional $J$ as:

$$
\begin{aligned}
J\left(\Gamma_{\boldsymbol{\theta}}, u_{f}\left(\Gamma_{\boldsymbol{\theta}}\right), u_{s}\left(\Gamma_{\boldsymbol{\theta}}\right)\right) & =\mathfrak{J}\left(\boldsymbol{\theta}, u_{f}\left(\Gamma_{\boldsymbol{\theta}}\right) \circ(I+\boldsymbol{\theta}), u_{s}\left(\Gamma_{\boldsymbol{\theta}}\right) \circ(I+\boldsymbol{\theta})\right) \\
& =\mathfrak{J}\left(\boldsymbol{\theta}, u_{f, \boldsymbol{\theta}}, u_{s, \boldsymbol{\theta}}\right),
\end{aligned}
$$

where we have denoted $u_{s, \boldsymbol{\theta}}:=u_{s}\left(\Gamma_{\boldsymbol{\theta}}\right) \circ(I+\boldsymbol{\theta})$ and $u_{f, \boldsymbol{\theta}}:=u_{f}\left(\Gamma_{\boldsymbol{\theta}}\right) \circ(I+\boldsymbol{\theta})$ the transported functions on the reference domain with the reference interface $\Gamma$. Identity (3.27) is the main motivation for introducing $\mathfrak{J}$ : indeed, as it is classical in shape optimization, the transported functions $u_{f, \boldsymbol{\theta}}, u_{s, \boldsymbol{\theta}}$ are differentiable with respect to $\boldsymbol{\theta}$ without additional regularity assumptions [32,40]. More precisely, the following lemma holds:

Lemma 2. The mappings $\boldsymbol{\theta} \mapsto u_{f, \boldsymbol{\theta}}$ and $\boldsymbol{\theta} \mapsto u_{s, \boldsymbol{\theta}}$, from $W_{0}^{1, \infty}\left(\Omega, \mathbb{R}^{d}\right)$ into $V_{f, 0}(\Gamma)$ and $V_{s}(\Gamma)$ are Fréchet differentiable at $\boldsymbol{\theta}=0$ and their Fréchet derivatives $\dot{u}_{f}(\boldsymbol{\theta})$ and $\dot{u}_{s}(\boldsymbol{\theta})$ in the direction $\boldsymbol{\theta}$ are the unique solutions to the following variational problems:

Find $\dot{u}_{f}(\boldsymbol{\theta}) \in V_{f, 0}(\Gamma)$ such that $\forall v_{f} \in V_{f, 0}(\Gamma)$,

$$
\int_{\Omega_{f}} \nu \nabla \dot{u}_{f}(\boldsymbol{\theta}) \cdot \nabla v_{f} \mathrm{~d} x=\int_{\Omega_{f}}\left(\operatorname{div}\left(f_{f} \boldsymbol{\theta}\right) v_{f}+\nu\left(\nabla \boldsymbol{\theta}+\nabla \boldsymbol{\theta}^{T}-\operatorname{div}(\boldsymbol{\theta}) I\right) \nabla u_{f} \cdot \nabla v_{f}\right) \mathrm{d} x,
$$

$$
\begin{aligned}
& \text { Find } \dot{u}_{s}(\boldsymbol{\theta}) \in V_{s}(\Gamma) \text { such that } \forall v_{s} \in V_{s}(\Gamma) \text {, } \\
& \int_{\Omega_{s}} \mu \nabla \dot{u}_{s}(\boldsymbol{\theta}) \cdot \nabla v_{s} \mathrm{~d} x=\int_{\Omega_{s}}\left(\operatorname{div}\left(f_{s} \boldsymbol{\theta}\right) v_{s}+\left(\nabla \boldsymbol{\theta}+\nabla \boldsymbol{\theta}^{T}-\operatorname{div}(\boldsymbol{\theta}) I\right) \mu \nabla u_{s} \cdot \nabla v_{s}\right) \mathrm{d} x-\int_{\Gamma} \nu \frac{\partial \dot{u}_{f}(\boldsymbol{\theta})}{\partial n} v_{s} \mathrm{~d} s
\end{aligned}
$$

where $-\frac{\partial \dot{u}_{f}(\boldsymbol{\theta})}{\partial n} \in H_{00}^{-1 / 2}(\Gamma)$ is defined for any $v \in H_{00}^{1 / 2}(\Gamma)$ by:

$$
-\int_{\Gamma} \nu \frac{\partial \dot{u}_{f}(\boldsymbol{\theta})}{\partial n} v \mathrm{~d} s=\int_{\Omega_{f}}\left(\operatorname{div}\left(f_{f} \boldsymbol{\theta}\right) \tilde{v}+\left(\nabla \boldsymbol{\theta}+\nabla \boldsymbol{\theta}^{T}-\operatorname{div}(\boldsymbol{\theta}) I\right) \nu \nabla u_{f} \cdot \nabla \tilde{v}\right) \mathrm{d} x-\int_{\Omega_{f}} \nu \nabla \dot{u}_{f}(\boldsymbol{\theta}) \cdot \nabla \tilde{v} \mathrm{~d} x
$$

for any extension $\tilde{v} \in V_{f}(\Gamma)$ of $v$ such that $v=\tilde{v}$ on $\Gamma$.

Proof. This is a classical result, so we content ourselves in briefly describing the main ideas of the proof. We first perform a change of variables in the variational formulations (3.8) and (3.11) so that the integrals involved there are written on fixed domains. Taking $v_{f} \circ(I+\boldsymbol{\theta})^{-1} \in V_{f, 0}\left(\Gamma_{\boldsymbol{\theta}}\right)$ as a test function in (3.8) for arbitrary $v_{f} \in V_{f, 0}(\Gamma)$, this yields a variational formulation for $u_{f, \boldsymbol{\theta}}$ :

$$
\forall v_{f} \in V_{f, 0}(\Gamma), \int_{\Omega_{f}} \nu A(\boldsymbol{\theta}) \nabla u_{f, \boldsymbol{\theta}} \cdot \nabla v_{f} \mathrm{~d} x=\int_{\Omega_{f}} f_{f} \circ(I+\boldsymbol{\theta}) v_{f}|\operatorname{det}(I+\nabla \boldsymbol{\theta})| \mathrm{d} x,
$$

where $A(\boldsymbol{\theta})$ is the $d \times d$ matrix $A(\boldsymbol{\theta})=|\operatorname{det}(I+\nabla \boldsymbol{\theta})|(I+\nabla \boldsymbol{\theta})^{-1}(I+\nabla \boldsymbol{\theta})^{-T}$. Note that $\Gamma$ corresponds to the reference configuration in (3.31) while it is the deformed configuration in (3.8).

Now, for a given $v_{s} \in V_{f}(\Gamma)$ and any extension $\tilde{v}_{s} \in V_{s}(\Gamma)$ such that $\tilde{v}_{s}=v_{s}$ on $\Gamma$, the function $\tilde{v}_{s} \circ(I+\boldsymbol{\theta})^{-1} \in V_{f}\left(\Gamma_{\boldsymbol{\theta}}\right)$ is an extension of $v_{s} \circ(I+\boldsymbol{\theta})^{-1} \in V_{s}\left(\Gamma_{\boldsymbol{\theta}}\right)$ satisfying $\tilde{v}_{s} \circ(I+\boldsymbol{\theta})^{-1}=v_{s} \circ(I+\boldsymbol{\theta})^{-1}$ on $\Gamma_{\boldsymbol{\theta}}$. Therefore taking $v_{s} \circ(I+\boldsymbol{\theta})^{-1}$ as a test function in (3.11) and performing a change of variables yields a variational formulation for $u_{s, \boldsymbol{\theta}}$ :

$$
\begin{array}{r}
\forall v_{s} \in V_{s}(\Gamma), \int_{\Omega_{s}} \mu A(\boldsymbol{\theta}) \nabla u_{s, \boldsymbol{\theta}} \cdot \nabla v_{s} \mathrm{~d} x=\int_{\Omega_{s}} f_{s} \circ(I+\boldsymbol{\theta}) \operatorname{det}|I+\nabla \boldsymbol{\theta}| \mathrm{d} x+\int_{\Omega_{f}} f_{f} \circ(I+\boldsymbol{\theta}) \tilde{v}_{s} \operatorname{det}|I+\nabla \boldsymbol{\theta}| \mathrm{d} x \\
-\int_{\Omega_{f}} \nu A(\boldsymbol{\theta}) \nabla u_{f, \boldsymbol{\theta}} \cdot \nabla \tilde{v}_{s} \mathrm{~d} x
\end{array}
$$

for any extension $\tilde{v}_{s} \in V_{f}(\Gamma)$ satisfying $\tilde{v}_{s}=v_{s}$ on $\Gamma$.

Eventually, a classical use of the implicit function theorem, as in [32], reveals that the mappings $\boldsymbol{\theta} \mapsto u_{f, \boldsymbol{\theta}}$ and $\boldsymbol{\theta} \mapsto u_{s, \boldsymbol{\theta}}$, from $W_{0}^{1, \infty}\left(\mathbb{R}^{d}, \mathbb{R}^{d}\right)$ into $V_{f, 0}(\Gamma)$ and $V_{s}(\Gamma)$ respectively, are Fréchet differentiable in the 
neighborhood of $\boldsymbol{\theta}=0$. Eqns. (3.28) and (3.29) are then simply obtained by differentiating (3.31) and (3.32) with respect to $\boldsymbol{\theta}$.

Remark 5. The functions $\dot{u}_{f}(\boldsymbol{\theta})$ and $\dot{u}_{s}(\boldsymbol{\theta})$, defined by Lemma 2, are the 'Lagrangian' derivatives of $u_{f}$ and $u_{s}$ with respect to variations of $\Gamma$, a notion of derivative which is directly compatible with the variational setting of the PDEs (3.1) and (3.2). Recall that $\dot{u}_{s}(\boldsymbol{\theta})$ and $\dot{u}_{f}(\boldsymbol{\theta})$ do not coincide with the perhaps more physical 'Eulerian' derivatives $u_{f}^{\prime}(\boldsymbol{\theta}), u_{s}^{\prime}(\boldsymbol{\theta})$, that are the differentials of the mappings $\boldsymbol{\theta} \mapsto u_{f}\left(\Gamma_{\boldsymbol{\theta}}\right)$ and $\boldsymbol{\theta} \mapsto u_{s}\left(\Gamma_{\boldsymbol{\theta}}\right)$ (without composition by $(I+\boldsymbol{\theta})$ ); see e.g. [40, 32, 1].

\subsubsection{Adjoint system and volume expression of the shape derivative.}

Assuming that the transported objective function $\mathfrak{J}$ has continuous partial derivatives at $\left(\boldsymbol{\theta}, \hat{u}_{s}, \hat{u}_{f}\right)=$ $\left(0, u_{s}(\Gamma), u_{f}(\Gamma)\right),(3.27)$ and the chain rule imply that the mapping $\boldsymbol{\theta} \mapsto J\left(\Gamma_{\boldsymbol{\theta}}, u_{f}\left(\Gamma_{\boldsymbol{\theta}}\right), u_{s}\left(\Gamma_{\boldsymbol{\theta}}\right)\right)$ is differentiable with respect to $\boldsymbol{\theta}$ and that its derivative at $\boldsymbol{\theta}=0$ reads:

$$
\frac{\mathrm{d}}{\mathrm{d} \boldsymbol{\theta}}\left[J\left(\Gamma_{\boldsymbol{\theta}}, u_{f}\left(\Gamma_{\boldsymbol{\theta}}\right), u_{s}\left(\Gamma_{\boldsymbol{\theta}}\right)\right)\right](\boldsymbol{\theta})=\frac{\mathrm{d}}{\mathrm{d} \boldsymbol{\theta}}\left[\mathfrak{J}\left(\boldsymbol{\theta}, u_{f, \boldsymbol{\theta}}, u_{s, \boldsymbol{\theta}}\right)\right](\boldsymbol{\theta})=\frac{\partial \mathfrak{J}}{\partial \boldsymbol{\theta}}(\boldsymbol{\theta})+\frac{\partial \mathfrak{J}}{\partial \hat{u}_{f}}\left(\dot{u}_{f}(\boldsymbol{\theta})\right)+\frac{\partial \mathfrak{J}}{\partial \hat{u}_{s}}\left(\dot{u}_{s}(\boldsymbol{\theta})\right) .
$$

Remark that, to keep notations as light as possible, we omit the point $\left(0, u_{f}(\Gamma), u_{s}(\Gamma)\right)$ where the partial derivatives of $\mathfrak{J}$ are evaluated in (3.33) and below. We now classically eliminate the occurrence of the Lagrangian derivatives $\dot{u}_{s}(\boldsymbol{\theta})$ and $\dot{u}_{f}(\boldsymbol{\theta})$ in (3.33) by introducing the adjoint states $p_{f} \in V_{f}(\Gamma)$ and $p_{s} \in V_{s}(\Gamma)$, defined in weak form by the following variational problems:

$$
\text { Find } p_{s} \in V_{s}(\Gamma) \text { such that } \forall v_{s} \in V_{s}(\Gamma), \int_{\Omega_{s}} \mu \nabla p_{s} \cdot \nabla v_{s} \mathrm{~d} x=\frac{\partial \mathfrak{J}}{\partial \hat{u}_{s}}\left(v_{s}\right),
$$

$$
\text { Find } p_{f} \in V_{f}(\Gamma) \text { such that } p_{f}=p_{s} \text { on } \Gamma \text { and } \forall v_{f} \in V_{f, 0}(\Gamma), \int_{\Omega_{f}} \nu \nabla p_{f} \cdot \nabla v_{f} \mathrm{~d} x=\frac{\partial \mathfrak{J}}{\partial \hat{u}_{f}}\left(v_{f}\right) \text {. }
$$

Of course, these adjoint states are exactly the same as the previous ones, defined by (3.18) and (3.19), if the objective function $J$ is defined by (3.17). However, (3.34) and (3.35) make sense for more general objective functions.

Proposition 2. Assume that the transported objective function $\mathfrak{J}$ given by (3.26) has continuous partial derivatives at $\left(\boldsymbol{\theta}, u_{s}, u_{f}\right)=\left(0, u_{s}(\Gamma), u_{f}(\Gamma)\right)$. Then, the mapping $\boldsymbol{\theta} \mapsto J\left(\Gamma_{\boldsymbol{\theta}}, u_{f}\left(\Gamma_{\boldsymbol{\theta}}\right), u_{s}\left(\Gamma_{\boldsymbol{\theta}}\right)\right)$, from $W_{0}^{1, \infty}\left(\Omega, \mathbb{R}^{d}\right)$ into $\mathbb{R}$, is Fréchet differentiable at $\boldsymbol{\theta}=0$ and its derivative reads:

$$
\begin{aligned}
& \frac{\mathrm{d}}{\mathrm{d} \boldsymbol{\theta}}\left[J\left(\Gamma_{\boldsymbol{\theta}}, u_{f}\left(\Gamma_{\boldsymbol{\theta}}\right), u_{s}\left(\Gamma_{\boldsymbol{\theta}}\right)\right)\right](\boldsymbol{\theta})=\frac{\partial \mathfrak{J}}{\partial \boldsymbol{\theta}}(\boldsymbol{\theta}) \\
&+\int_{\Omega_{f}}\left[\operatorname{div}\left(f_{f} \boldsymbol{\theta}\right) p_{f}+\right.\left.\left(\nabla \boldsymbol{\theta}+\nabla \boldsymbol{\theta}^{T}-\operatorname{div}(\boldsymbol{\theta}) I\right) \nu \nabla u_{f} \cdot \nabla p_{f}\right] \mathrm{d} x \\
& \quad+\int_{\Omega_{s}}\left[\operatorname{div}\left(f_{s} \boldsymbol{\theta}\right) p_{s}+\left(\nabla \boldsymbol{\theta}+\nabla \boldsymbol{\theta}^{T}-\operatorname{div}(\boldsymbol{\theta}) I\right) \mu \nabla u_{s} \cdot \nabla p_{s}\right] \mathrm{d} x,
\end{aligned}
$$

where $p_{f}$ and $p_{s}$ are the adjoint states defined by (3.34) and (3.35).

Remark 6. Since formula (3.36) for the shape derivative of $J$ involves bulk integrals, it is called a volume expression of the shape derivative. In the next subsection, it is shown that it can equivalently be written in terms of surface integrals (this is the so-called Hadamard structure theorem).

Proof. We first take $v_{s}=\dot{u}_{s}(\boldsymbol{\theta})$ and $v_{f}=\dot{u}_{f}(\boldsymbol{\theta})$ in the adjoint equations (3.34) and (3.35) to obtain:

$$
\begin{gathered}
\frac{\partial \mathfrak{J}}{\partial \hat{u}_{s}}\left(\dot{u}_{s}(\boldsymbol{\theta})\right)=\int_{\Omega_{s}} \mu \nabla p_{s} \cdot \nabla \dot{u}_{s}(\boldsymbol{\theta}) \mathrm{d} x, \\
\frac{\partial \mathfrak{J}}{\partial \hat{u}_{f}}\left(\dot{u}_{f}(\boldsymbol{\theta})\right)=\int_{\Omega_{f}} \nu \nabla p_{f} \cdot \nabla \dot{u}_{f}(\boldsymbol{\theta}) \mathrm{d} x .
\end{gathered}
$$


Then taking $v_{s}=p_{s}$ in the variational formulation (3.29) for $\dot{u}_{s}(\boldsymbol{\theta})$ yields:

$$
\int_{\Omega_{s}} \mu \nabla p_{s} \cdot \nabla \dot{u}_{s}(\boldsymbol{\theta}) \mathrm{d} x=\int_{\Omega_{s}}\left(\operatorname{div}\left(f_{s} \boldsymbol{\theta}\right) p_{s}+\left(\nabla \boldsymbol{\theta}+\nabla \boldsymbol{\theta}^{T}-\operatorname{div}(\boldsymbol{\theta}) I\right) \mu \nabla u_{s} \cdot \nabla p_{s}\right) \mathrm{d} x-\int_{\Gamma} \nu \frac{\partial \dot{u}_{f}(\boldsymbol{\theta})}{\partial n} p_{s} \mathrm{~d} s .
$$

Now remarking that $\tilde{v}=p_{f} \in V_{f}(\Gamma)$ is an extension of $v=p_{s}=p_{f} \in H_{00}^{1 / 2}(\Gamma)$, taking $\tilde{v}=p_{f}$ in $(3.30)$ implies:

$$
\int_{\Omega_{f}} \nu \nabla p_{f} \cdot \nabla \dot{u}_{f}(\boldsymbol{\theta}) \mathrm{d} x=\int_{\Omega_{f}}\left(\operatorname{div}\left(f_{f} \boldsymbol{\theta}\right) p_{f}+\left(\nabla \boldsymbol{\theta}+\nabla \boldsymbol{\theta}^{T}-\operatorname{div}(\boldsymbol{\theta}) I\right) \nu \nabla u_{f} \cdot \nabla p_{f}\right) \mathrm{d} x+\int_{\Gamma} \nu \frac{\partial \dot{u}_{f}(\boldsymbol{\theta})}{\partial n} p_{s} \mathrm{~d} s .
$$

The desired formula (3.36) follows then by summation of (3.39) and (3.40).

3.4.3. Obtaining the surface expression of the shape derivative.

Hadamard's structure theorem states that under little additional regularity on the optimized interface $\Gamma$ and on the considered vector fields $\boldsymbol{\theta}$, the shape derivative of a 'regular enough' objective function $J$ depends only on the normal component $\boldsymbol{\theta} \cdot \boldsymbol{n}$ on $\Gamma$; see [32, 24] for precise statements. In this section, we review how this remark allows to obtain a surface expression for the shape derivative of $J$ from the volume expression (3.36) in a simple way. To achieve this, we classically rely on three 'regularity assumptions', which we assume to be satisfied throughout this section:

(1) The considered deformations $\boldsymbol{\theta}$ are smooth, e.g. of class $\mathcal{C}^{1}$;

(2) The state and adjoint variables $u_{s}, u_{f}, p_{s}, p_{f}$ enjoy $H^{2}$ regularity in their domain of definition; this is for instance the case when $\Omega$ and $\Gamma$ are smooth enough; see e.g. [12].

(3) The partial derivative $\frac{\partial \mathfrak{J}}{\partial \boldsymbol{\theta}}$ is sufficiently 'regular', in the sense that there exist $\boldsymbol{f}_{\mathfrak{J}} \in L^{1}\left(\Omega, \mathbb{R}^{d}\right)$ and $\boldsymbol{g}_{\mathfrak{J}} \in L^{1}\left(\Gamma, \mathbb{R}^{d}\right)$ such that

$$
\forall \boldsymbol{\theta} \in W_{0}^{1, \infty}\left(\Omega, \mathbb{R}^{d}\right), \frac{\partial \mathfrak{J}}{\partial \boldsymbol{\theta}}(\boldsymbol{\theta})=\int_{\Omega} \boldsymbol{f}_{\mathfrak{J}} \cdot \boldsymbol{\theta} \mathrm{d} x+\int_{\Gamma} \boldsymbol{g}_{\mathfrak{J}} \cdot \boldsymbol{\theta} \mathrm{d} s .
$$

Note that the uniqueness of the decomposition (3.41), when it exists, is straightforward. The existence of such a structure is typically obtained by performing integration by parts on $\frac{\partial \mathfrak{J}}{\partial \theta}$ using, in turn, the $H^{2}$ regularity of the state and adjoint variables $u_{s}, u_{f}, p_{s}, p_{f}$.

In the following and under these assumptions, we denote by

$$
\forall \boldsymbol{\theta} \in W_{0}^{1, \infty}\left(\Omega, \mathbb{R}^{d}\right), \quad \frac{\overline{\partial \mathfrak{J}}}{\partial \boldsymbol{\theta}}(\boldsymbol{\theta}):=\int_{\Gamma}\left(\boldsymbol{g}_{\mathfrak{J}} \cdot \boldsymbol{n}\right)(\boldsymbol{\theta} \cdot \boldsymbol{n}) \mathrm{d} s,
$$

the part of $\frac{\partial \mathfrak{J}}{\partial \boldsymbol{\theta}}$ that depends only on $\boldsymbol{\theta} \cdot \boldsymbol{n}$. The main result of this section is the following proposition:

Proposition 3. Under the above assumptions, the shape derivative (3.36) rewrites as an integral over the boundary $\Gamma$ involving only the normal component $\boldsymbol{\theta} \cdot \boldsymbol{n}$ :

$$
\begin{aligned}
\frac{\mathrm{d}}{\mathrm{d} \boldsymbol{\theta}}\left[J\left(\Gamma_{\boldsymbol{\theta}}, u_{f}\left(\Gamma_{\boldsymbol{\theta}}\right), u_{s}\left(\Gamma_{\boldsymbol{\theta}}\right)\right)\right](\boldsymbol{\theta}) & =\frac{\overline{\partial \mathfrak{J}}}{\partial \boldsymbol{\theta}}(\boldsymbol{\theta}) \\
& +\int_{\Gamma}\left[f_{f} p_{f}-f_{s} p_{s}-\nu \nabla u_{f} \cdot \nabla p_{f}+\mu \nabla u_{s} \cdot \nabla p_{s}+2 \nu \frac{\partial u_{f}}{\partial n} \frac{\partial p_{f}}{\partial n}-2 \mu \frac{\partial u_{s}}{\partial n} \frac{\partial p_{s}}{\partial n}\right](\boldsymbol{\theta} \cdot \boldsymbol{n}) \mathrm{d} s .
\end{aligned}
$$

Proof. The regularity assumptions make it possible to perform integration by parts in the volume expression (3.36), which yields:

$$
\begin{aligned}
& \frac{\mathrm{d}}{\mathrm{d} \boldsymbol{\theta}}\left[J\left(\Gamma_{\boldsymbol{\theta}}, u_{f}\left(\Gamma_{\boldsymbol{\theta}}\right), u_{s}\left(\Gamma_{\boldsymbol{\theta}}\right)\right)\right](\boldsymbol{\theta}) \\
& =\int_{\Gamma} \boldsymbol{g}_{\mathfrak{J}} \cdot \boldsymbol{\theta} \mathrm{d} s+\int_{\Gamma}\left[f_{f} p_{f}(\boldsymbol{\theta} \cdot \boldsymbol{n})+\nu\left(\boldsymbol{\theta} \cdot \nabla u_{f}\right)\left(\nabla p_{f} \cdot \boldsymbol{n}\right)+\nu\left(\boldsymbol{n} \cdot \nabla u_{f}\right)\left(\nabla p_{f} \cdot \boldsymbol{\theta}\right)-\nu\left(\nabla u_{f} \cdot \nabla p_{f}\right)(\boldsymbol{\theta} \cdot \boldsymbol{n})\right] \mathrm{d} s \\
& \quad-\int_{\Gamma}\left[f_{s} p_{s}(\boldsymbol{\theta} \cdot \boldsymbol{n})+\mu\left(\boldsymbol{\theta} \cdot \nabla u_{s}\right)\left(\nabla p_{s} \cdot \boldsymbol{n}\right)+\mu\left(\boldsymbol{n} \cdot \nabla u_{s}\right)\left(\nabla p_{s} \cdot \boldsymbol{\theta}\right)-\mu\left(\nabla u_{s} \cdot \nabla p_{s}\right)(\boldsymbol{\theta} \cdot \boldsymbol{n})\right] \mathrm{d} s \\
& +\int_{\Omega} \boldsymbol{\Lambda} \cdot \boldsymbol{\theta} \mathrm{d} x,
\end{aligned}
$$


for some function $\boldsymbol{\Lambda} \in L^{1}\left(\Omega, \mathbb{R}^{d}\right)$. Hadamard's structure theorem implies that (3.44) must vanish for vector fields $\boldsymbol{\theta}$ compactly supported in $\Omega_{s}$ or in $\Omega_{f}$, or for vector fields $\boldsymbol{\theta}$ which are tangential to $\Gamma$. Therefore, $\boldsymbol{\Lambda}=0$, and one obtains (3.43) by computing (3.44) with $\boldsymbol{\theta}$ normal to $\Gamma$.

Formula (3.43) is called a surface expression of the shape derivative of $J$. It is easily verified that (3.43) coincides with (3.21) in the particular case where the objective functional $J$ has the form (3.17) considered previously. An asset of the above Lagrangian method is that it depends neither on the nature of the objective function $J$, nor on the type of boundary conditions satisfied by the state variables $u_{f}$ and $u_{s}$. Both expressions (3.36) and (3.43) are convenient to implement because they require minimal inputs from the user: namely, the expression of the partial derivatives of $\mathfrak{J}$ with respect to $\boldsymbol{\theta}$ (for (3.36) and (3.43)) and the state variables $u_{s}, u_{f}$ (for solving the adjoint system (3.28) and (3.29)).

Remark 7. As emphasized in e.g. [29, 57], the volume expression (3.36) requires less regularity on the data (only the one obtained from the variational setting) than (3.43). For example, it is well-known that $u_{f}, u_{s}$ may fail to be $H^{2}$ functions when the domains $\Omega_{s}$ or $\Omega_{f}$ involve corners [12]; in such case (3.36) remains valid while the surface expression (3.43) may become invalid. Furthermore, some authors have found evidence that the Fréchet derivatives of shape functional are better approximated when discretizing the volume form [33]. Nevertheless, one should note that because $W_{0}^{1, \infty}\left(\Omega, \mathbb{R}^{d}\right)$ is not a Hilbert space, regularity assumptions are still required in the end for the identification (3.15) of the Fréchet derivative to a gradient.

Let us now illustrate these results with the calculation of the shape derivative of the 'solid compliance', which is not of the form (3.17) considered previously:

$$
J\left(\Gamma, u_{f}(\Gamma), u_{s}(\Gamma)\right)=\int_{\Omega_{s}} \mu\left|\nabla u_{s}\right|^{2} \mathrm{~d} x
$$

The associated transported objective function $\mathfrak{J}$ via (3.26) reads:

$$
\mathfrak{J}\left(\boldsymbol{\theta}, \hat{u}_{f}, \hat{u}_{s}\right)=\int_{(I+\boldsymbol{\theta}) \Omega_{s}} \mu\left|\nabla\left(\hat{u}_{s} \circ(I+\boldsymbol{\theta})^{-1}\right)\right|^{2} \mathrm{~d} x=\int_{\Omega_{s}} \mu\left|(I+\nabla \boldsymbol{\theta})^{-T} \nabla \hat{u}_{s}\right|^{2}|\operatorname{det}(I+\boldsymbol{\theta})| \mathrm{d} x .
$$

Lemma 3. The functional $\mathfrak{J}$ defined in (3.46) has continuous partial derivatives at $\left(\boldsymbol{\theta}, \hat{u}_{s}, \hat{u}_{f}\right)=\left(0, u_{s}(\Gamma), u_{f}(\Gamma)\right)$ which are given by:

$$
\begin{gathered}
\frac{\partial \mathfrak{J}}{\partial \hat{u}_{f}}\left(v_{f}\right)=0, \quad \frac{\partial \mathfrak{J}}{\partial \hat{u}_{s}}\left(v_{s}\right)=\int_{\Omega_{s}} 2 \mu \nabla u_{s} \cdot \nabla v_{s} \mathrm{~d} x, \\
\frac{\partial \mathfrak{J}}{\partial \boldsymbol{\theta}}(\boldsymbol{\theta})=\int_{\Omega_{s}}\left(\mu\left|\nabla u_{s}\right|^{2} \operatorname{div}(\boldsymbol{\theta})-2 \mu \nabla u_{s} \cdot \nabla \boldsymbol{\theta} \cdot \nabla u_{s}\right) \mathrm{d} x, \\
\frac{\overline{\partial \mathfrak{J}}}{\partial \boldsymbol{\theta}}(\boldsymbol{\theta})=\int_{\Gamma}\left[-\mu\left|\nabla u_{s}\right|^{2}+2 \mu\left|\frac{\partial u_{s}}{\partial n}\right|^{2}\right](\boldsymbol{\theta} \cdot \boldsymbol{n}) \mathrm{d} s .
\end{gathered}
$$

Therefore the solid compliance (3.45) $\boldsymbol{\theta} \mapsto J\left(\Gamma_{\boldsymbol{\theta}}, u_{s}\left(\Gamma_{\boldsymbol{\theta}}\right), u_{f}\left(\Gamma_{\boldsymbol{\theta}}\right)\right)$ is differentiable with respect to $\boldsymbol{\theta} \in W_{0}^{1, \infty}\left(\Omega, \mathbb{R}^{d}\right)$, and the shape derivative is given by Proposition 2 in volume form or Proposition 3 in surface form.

Remark 8. Note that in this particular case the adjoint state $p_{s}$ defined by (3.34) satisfies $p_{s}=2 u_{s}$, but no such property holds for $p_{f}$ (the problem is not self-adjoint). Unlike the usual case of the standard Poisson equation, there is no obvious way to write the solid compliance (3.45) in a form (3.17) involving only volumic integrals in $u_{s}, u_{f}$ without occurrence of their gradient as in Section 3.3. For example, the formulation as the work of external forces,

$$
J\left(\Gamma, u_{s}(\Gamma), u_{f}(\Gamma)\right)=\int_{\Omega_{s}} f_{s} u_{s} \mathrm{~d} x-\int_{\Gamma} \nu \frac{\partial u_{f}}{\partial n} u_{s} \mathrm{~d} s,
$$

involves the gradient of $u_{f}$; it is therefore less convenient to handle than (3.45), since the partial derivative

$$
\frac{\partial \mathfrak{J}}{\partial u_{f}}: v \mapsto-\int_{\Gamma} \nu \frac{\partial v}{\partial n} u_{s} \mathrm{~d} s
$$

is not defined on $V_{f, 0}$, but merely in a subspace of smoother functions. 


\subsubsection{A mixed variational formulation for the state and adjoint problem.}

We conclude this section with an alternative point of view which sheds some light on the a priori 'surprising' boundary condition $p_{s}=p_{f}$ on $\Gamma$ for the adjoint system (3.34) and (3.35). The starting observation is that the systems (3.1) and (3.2) may be formally described by means of a variational formulation for the couple $\left(u_{s}, u_{f}\right)$ which features different functional spaces for the solution and test functions (a so-called Petrov-Galerkin variational formulation). The latter is simply obtained by summing (3.8) and (3.11):

$$
\begin{aligned}
& \text { Find }\left(u_{f}, u_{s}\right) \in H_{0}^{1}\left(\Omega_{f}\right) \times H^{1}\left(\Omega_{s}\right) \text { such that } \forall v \in H^{1}(\Omega) \text {, } \\
& \qquad \int_{\Omega_{s}} \mu \nabla u_{s} \cdot \nabla v \mathrm{~d} x+\int_{\Omega_{f}} \nu \nabla u_{f} \cdot \nabla v \mathrm{~d} x=\int_{\Omega_{s}} f_{s} v \mathrm{~d} x+\int_{\Omega_{f}} f_{f} v \mathrm{~d} x .
\end{aligned}
$$

Problem (3.51) implicitly encloses the transmission condition $\mu \frac{\partial u_{s}}{\partial n}=\nu \frac{\partial u_{f}}{\partial n}$ on $\Gamma$, and the need to resort to extensions of test functions defined on $\Omega_{s}$ in the variational formulation (3.11) for $u_{s}$ is reflected here in that the test function $v$ belongs to $H^{1}(\Omega)$.

As is customary (see for instance Section 3.3), the adjoint system is obtained by formally taking the linear transpose of the mixed variational formulation (3.51) (with a different right-hand side), which exchanges the roles of the functional spaces for the sought solution and the test functions:

$$
\begin{aligned}
& \text { Find } p \in H^{1}(\Omega) \text { such that } \forall\left(v_{f}, v_{s}\right) \in H_{0}^{1}\left(\Omega_{f}\right) \times H^{1}\left(\Omega_{s}\right) \text {, } \\
& \qquad \int_{\Omega_{s}} \nu \nabla p \cdot \nabla v_{s} \mathrm{~d} x+\int_{\Omega_{f}} \nu \nabla p \cdot \nabla v_{f} \mathrm{~d} x=\frac{\partial \mathfrak{J}}{\partial \hat{u}_{s}}\left(v_{s}\right)+\frac{\partial \mathfrak{J}}{\partial \hat{u}_{f}}\left(v_{f}\right) .
\end{aligned}
$$

The above equation is in turn equivalent to the triangular system (3.28) and (3.29) for the restrictions $p_{s}$ and $p_{f}$ of $p$ on $\Omega_{s}$ and $\Omega_{f}$, where the transmission condition $p_{s}=p_{f}$ on $\Gamma$ is implicitly contained in the requirement that $p$ be an element of $H^{1}(\Omega)$.

Remark 9. The above argument is only formal insofar as there is no guarantee that the variational problem (3.51) be well-posed. It can however be made rigorous by changing the functional spaces featured in there, more precisely, by searching for $\left(u_{f}, u_{s}\right)$ in

$$
\left\{\left(u_{f}, u_{s}\right) \in H_{0}^{1}\left(\Omega_{f}\right) \times H^{1}\left(\Omega_{s}\right), \mathbb{1}_{\Omega_{f}} \nu \nabla u_{f}+\mathbb{1}_{\Omega_{s}} \nu \nabla u_{s} \in H(\operatorname{div}, \Omega)\right\},
$$

where $\mathbb{1}_{\Omega_{f}}$ (resp. $\mathbb{1}_{\Omega_{s}}$ ) is the characteristic function of $\Omega_{f}$ (resp. $\Omega_{s}$ ), and by searching for $v$ in $L^{2}(\Omega)$. It is possible to prove that the inf-sup condition of the Banach-Necas-Babuska theorem holds (see [26]) in the case of this new version of (3.51), which guarantees its well-posedness.

\section{Shape Derivatives For the three-Physic PROBlem}

We now briefly describe how the methodology presented in Section 3 applies to the weakly coupled, multiphysics system (2.1) to (2.3). Let us introduce the functional spaces which are required, respectively, for the Navier-Stokes equations

$$
V_{\boldsymbol{v}, p}(\Gamma)=\left\{(\boldsymbol{w}, q) \in H^{1}\left(\Omega_{f}, \mathbb{R}^{d}\right) \times L^{2}\left(\Omega_{f}\right) / \mathbb{R} \mid \boldsymbol{w}=0 \text { on } \partial \Omega_{f}\right\},
$$

for the thermal equation

$$
V_{T}(\Gamma)=\left\{S \in H^{1}(\Omega) \mid S=0 \text { on } \partial \Omega_{T}^{D}\right\},
$$

for the thermo-mechanical equations

$$
V_{\boldsymbol{u}}(\Gamma)=\left\{\boldsymbol{r} \in H^{1}\left(\Omega_{s}, \mathbb{R}^{d}\right) \mid \boldsymbol{r}=0 \text { on } \partial \Omega_{s}^{D}\right\} .
$$

Note that, as is customary in the theory of the Navier-Stokes equations, the quotient space $L^{2}\left(\Omega_{f}\right) / \mathbb{R}$, associated to the pressure field, gathers square integrable functions defined up to an additive constant. We consider as well the affine spaces associated to the non-homogeneous Dirichlet boundary data $\boldsymbol{v}_{0} \in$ 
$H^{1 / 2}\left(\partial \Omega_{f}^{D}, \mathbb{R}^{d}\right), \boldsymbol{u}_{0} \in H^{1 / 2}\left(\partial \Omega_{s}^{D}, \mathbb{R}^{d}\right)$ and $T_{0} \in H^{1 / 2}\left(\partial \Omega_{T}^{D}\right)$ featured in (2.1) to (2.3):

$$
\begin{aligned}
\boldsymbol{v}_{0}+V_{\boldsymbol{v}, p}(\Gamma) & =\left\{(\boldsymbol{w}, q) \in H^{1}\left(\Omega_{f}, \mathbb{R}^{d}\right) \times L^{2}\left(\Omega_{f}\right) / \mathbb{R} \mid \boldsymbol{w}=\boldsymbol{v}_{0} \text { on } \partial \Omega_{f}^{D} \text { and } \boldsymbol{w}=0 \text { on } \Gamma\right\}, \\
T_{0}+V_{T}(\Gamma) & =\left\{S \in H^{1}(\Omega) \mid S=T_{0} \text { on } \partial \Omega_{T}^{D}\right\}, \\
\boldsymbol{u}_{0}+V_{\boldsymbol{u}}(\Gamma) & =\left\{\boldsymbol{r} \in H^{1}\left(\Omega_{s}, \mathbb{R}^{d}\right) \mid \boldsymbol{r}=\boldsymbol{u}_{0} \text { on } \partial \Omega_{s}^{D}\right\} .
\end{aligned}
$$

Finally, we shall make use of the trace space

$$
H_{00}^{1 / 2}\left(\Gamma, \mathbb{R}^{d}\right)=\left\{\left.\boldsymbol{r}\right|_{\Gamma} \mid \boldsymbol{r} \in H^{1}\left(\Omega_{f}, \mathbb{R}^{d}\right) \text { and } \boldsymbol{r}=0 \text { on } \partial \Omega_{f} \backslash \Gamma\right\},
$$

and its dual $H_{00}^{-1 / 2}\left(\Gamma, \mathbb{R}^{d}\right)$. The state variables $\boldsymbol{v}, p, T, \boldsymbol{u}$ are the solutions to the following variational problems. For Navier-Stokes equations $(2.1)$, find $(\boldsymbol{v}, p) \in \boldsymbol{v}_{0}+V_{\boldsymbol{v}, p}(\Gamma)$ such that

$$
\forall(\boldsymbol{w}, q) \in V_{\boldsymbol{v}, p}(\Gamma) \quad \int_{\Omega_{f}}\left[\sigma_{f}(\boldsymbol{v}, p): \nabla \boldsymbol{w}+\rho \boldsymbol{w} \cdot \nabla \boldsymbol{v} \cdot \boldsymbol{v}-q \operatorname{div}(\boldsymbol{v})\right] \mathrm{d} x=\int_{\Omega_{f}} \boldsymbol{f}_{f} \cdot \boldsymbol{w} \mathrm{d} x ;
$$

For the thermal equation (2.2), find $T \in T_{0}+V_{T}(\Gamma)$ such that, for any $S \in V_{T}(\Gamma)$,

$$
\int_{\Omega_{s}} k_{s} \nabla T \cdot \nabla S \mathrm{~d} x+\int_{\Omega_{f}}\left(k_{f} \nabla T \cdot \nabla S+\rho c_{p} S \boldsymbol{v} \cdot \nabla T\right) \mathrm{d} x=\int_{\Omega_{s}} Q_{s} S \mathrm{~d} x+\int_{\Omega_{f}} Q_{f} S \mathrm{~d} x+\int_{\partial \Omega_{T}^{N}} h S \mathrm{~d} s ;
$$

For the thermo-mechanical equations (2.3), find $\boldsymbol{u} \in \boldsymbol{u}_{0}+V_{\boldsymbol{u}}(\Gamma)$ such that

$$
\forall \boldsymbol{r} \in V_{\boldsymbol{u}}(\Gamma), \quad \int_{\Omega_{s}} \sigma_{s}\left(\boldsymbol{u}, T_{s}\right): \nabla \boldsymbol{r} \mathrm{d} x=\int_{\Omega_{s}} \boldsymbol{f}_{s} \cdot \boldsymbol{r} \mathrm{d} x+\int_{\partial \Omega_{s}^{N}} \boldsymbol{g} \cdot \boldsymbol{r} \mathrm{d} s-\int_{\Gamma} \boldsymbol{r} \cdot \sigma_{f}(\boldsymbol{v}, p) \cdot \boldsymbol{n} \mathrm{d} s .
$$

Let us comment on the well-posedness of the coupled system of variational problems (4.2), (4.3) and (4.4). As in Section 3, the volumic source terms are assumed to enjoy $H^{1}$ regularity in their domain: $\boldsymbol{f}_{f} \in$ $H^{1}\left(\Omega_{f}, \mathbb{R}^{d}\right), \boldsymbol{f}_{s} \in H^{1}\left(\Omega_{s}, \mathbb{R}^{d}\right), Q_{f} \in H^{1}\left(\Omega_{f}\right), Q_{s} \in H^{1}\left(\Omega_{s}\right)$. The surface fluxes $h, \boldsymbol{g}$ are assumed to belong to $L^{2}$ spaces. At first, the classical theory for the Navier-Stokes equation states that (4.2) is well-posed as soon as the Reynolds numbers Re $:=\left\|\boldsymbol{v}_{0}\right\|_{H^{1 / 2}\left(\partial \Omega_{f}^{D}, \mathbb{R}^{d}\right)} \rho / \nu$ is sufficiently small; see [55].

The variational formulation (4.3) of the thermal problem is not well-posed in utter generality because of the lack of coercivity induced by the advection term $\int_{\Omega_{f}} \rho c_{p} S \boldsymbol{v} \cdot \nabla T \mathrm{~d} x$ and of the presence of inhomogeneous Dirichlet boundary conditions. However, in usual applications [44, 38], it is customary to impose a Dirichlet boundary condition $T=T_{0, f}$ at the inlet of the computational domain (i.e. where $\boldsymbol{v} \cdot \boldsymbol{n}<0$ ) and a Neumann boundary condition $-k_{f} \nabla T \cdot \boldsymbol{n}=0$ at the outlet $(\boldsymbol{v} \cdot \boldsymbol{n}>0)$. This together with the incompressibility condition $\operatorname{div}(\boldsymbol{v})=0$ is easily shown to imply the coercivity of the bilinear form featured in (4.3); see e.g. [11].

Eventually, the well-posedness of the linear elasticity problem (4.4) results from the Lax-Milgram theorem, the only subtle point is that, as in Section $3, \sigma_{f}(\boldsymbol{v}, p) \cdot \boldsymbol{n}$ is an element of the dual space of $H_{00}^{1 / 2}\left(\Gamma, \mathbb{R}^{d}\right)$ : if $\boldsymbol{v}, p$ were regular enough, the following integration by parts would hold true:

$$
\forall \boldsymbol{r} \in V_{\boldsymbol{u}}(\Gamma),-\int_{\Gamma} \boldsymbol{r} \cdot \sigma_{f}(\boldsymbol{v}, p) \cdot \boldsymbol{n} \mathrm{d} s=\int_{\Omega_{f}}\left(-\operatorname{div}\left(\sigma_{f}(\boldsymbol{v}, p)\right) \cdot \boldsymbol{r}-\sigma_{f}(\boldsymbol{v}, p): \nabla \boldsymbol{r}\right) \mathrm{d} x .
$$

Hence the normal stress $\sigma_{f}(\boldsymbol{v}, p) \boldsymbol{n}$ can be understood mathematically as an element of $H_{00}^{-1 / 2}\left(\Gamma, \mathbb{R}^{d}\right)$, defined by

$$
\forall \boldsymbol{r} \in H_{00}^{1 / 2}\left(\Gamma, \mathbb{R}^{d}\right),-\int_{\Gamma} \boldsymbol{r} \cdot \sigma_{f}(\boldsymbol{v}, p) \cdot \boldsymbol{n} \mathrm{d} s=\int_{\Omega_{f}}\left(\boldsymbol{f}_{f} \cdot \tilde{\boldsymbol{r}}-\rho \tilde{\boldsymbol{r}} \cdot \nabla \boldsymbol{v} \cdot \boldsymbol{v}-\sigma_{f}(\boldsymbol{v}, p): \nabla \tilde{\boldsymbol{r}}+\tilde{q} \operatorname{div}(\boldsymbol{v})\right) \mathrm{d} x
$$

for any extension $(\tilde{\boldsymbol{r}}, \tilde{q}) \in H^{1}\left(\Omega_{f}, \mathbb{R}^{d}\right) \times\left(L^{2}\left(\Omega_{f}\right) / \mathbb{R}\right)$ satisfying $\tilde{\boldsymbol{r}}=\boldsymbol{r}$ on $\Gamma$. Note that although it is not fully necessary, we consider also an extension $\tilde{q}$ of the pressure field to maintain a complete analogy with (3.10), which turns to be convenient in the calculation of the shape derivative performed in Appendix A.

Throughout this section, we assume that the above conditions for the well-posedness of the coupled system of variational problems (4.2), (4.3) and (4.4) are fulfilled.

In the above context, we aim at solving the minimization problem

$$
\min _{\Gamma} J(\Gamma, \boldsymbol{v}(\Gamma), p(\Gamma), T(\Gamma), \boldsymbol{u}(\Gamma)),
$$


where the velocity $\boldsymbol{v}(\Gamma)$, pressure $p(\Gamma)$, temperature $T(\Gamma)$ and elastic displacement $\boldsymbol{u}(\Gamma)$ are the solutions to (2.1) to $(2.3)$.

In order to compute shape derivatives with respect to variations of a given interface $\Gamma$, we introduce as in Section 3.4 .2 a transported functional $\mathfrak{J}$ defined on the fixed functional space $W_{0}^{1, \infty}\left(\Omega, \mathbb{R}^{d}\right) \times H^{1}\left(\Omega_{f}, \mathbb{R}^{d}\right) \times$ $\left(L^{2}\left(\Omega_{f}\right) / \mathbb{R}\right) \times H^{1}(\Omega) \times H^{1}\left(\Omega_{s}, \mathbb{R}^{d}\right)$ by:

$$
\begin{aligned}
\forall \boldsymbol{\theta} \in W_{0}^{1, \infty}\left(\Omega, \mathbb{R}^{d}\right),(\hat{\boldsymbol{v}}, \hat{p}, \hat{T}, \hat{\boldsymbol{u}}) \in H^{1}\left(\Omega_{f}, \mathbb{R}^{d}\right) \times\left(L^{2}\left(\Omega_{f}\right) / \mathbb{R}\right) \times H^{1}(\Omega) \times H^{1}\left(\Omega_{s}, \mathbb{R}^{d}\right) \\
\mathfrak{J}(\boldsymbol{\theta}, \hat{\boldsymbol{v}}, \hat{p}, \hat{T}, \hat{\boldsymbol{u}})=J\left(\Gamma_{\boldsymbol{\theta}}, \hat{\boldsymbol{v}} \circ(I+\boldsymbol{\theta})^{-1}, \hat{p} \circ(I+\boldsymbol{\theta})^{-1}, \hat{T} \circ(I+\boldsymbol{\theta})^{-1}, \hat{\boldsymbol{u}} \circ(I+\boldsymbol{\theta})^{-1}\right) .
\end{aligned}
$$

The only requirement made on $J$ is that the associated functional $\mathfrak{J}$ has continuous partial derivatives at $(\boldsymbol{\theta}, \hat{\boldsymbol{v}}, \hat{p}, \hat{T}, \hat{\boldsymbol{u}})=(0, \boldsymbol{v}(\Gamma), p(\Gamma), T(\Gamma), \boldsymbol{u}(\Gamma))$. Under this assumption, arguing as in Section 3.4.2 and Section 3.3, we define the adjoint variables $\boldsymbol{w}, q, S, \boldsymbol{r}$ as follows. The elasticity adjoint variable $\boldsymbol{r} \in V_{\boldsymbol{u}}(\Gamma)$ is the solution of

$$
\int_{\Omega_{s}} A e(\boldsymbol{r}): \nabla \boldsymbol{r}^{\prime} \mathrm{d} x=\frac{\partial \mathfrak{J}}{\partial \hat{\boldsymbol{u}}}\left(\boldsymbol{r}^{\prime}\right) \quad \forall \boldsymbol{r}^{\prime} \in V_{\boldsymbol{u}}(\Gamma)
$$

The thermal adjoint variable $S \in V_{T}(\Gamma)$ is the solution of

$$
\int_{\Omega_{s}} k_{s} \nabla S \cdot \nabla S^{\prime} \mathrm{d} x+\int_{\Omega_{f}}\left(k_{f} \nabla S \cdot \nabla S^{\prime}+\rho c_{p} S \boldsymbol{v} \cdot \nabla S^{\prime}\right) \mathrm{d} x=\int_{\Omega_{s}} \alpha \operatorname{div}(\boldsymbol{r}) S^{\prime} \mathrm{d} x+\frac{\partial \mathfrak{J}}{\partial \hat{T}}(S) \quad \forall S^{\prime} \in V_{T}(\Gamma)
$$

The fluid adjoint variables $(\boldsymbol{w}, q) \in H^{1}\left(\Omega_{f}, \mathbb{R}^{d}\right) \times\left(L^{2}\left(\Omega_{f}\right) / \mathbb{R}\right)$ are the solution of

$$
\begin{aligned}
& \boldsymbol{w}=\boldsymbol{r} \text { on } \Gamma \text { and } \forall\left(\boldsymbol{w}^{\prime}, q^{\prime}\right) \in V_{\boldsymbol{v}, p}(\Gamma) \\
& \qquad \int_{\Omega_{f}}\left(\sigma_{f}(\boldsymbol{w}, q): \nabla \boldsymbol{w}^{\prime}+\rho \boldsymbol{w} \cdot \nabla \boldsymbol{w}^{\prime} \cdot \boldsymbol{v}+\rho \boldsymbol{w} \cdot \nabla \boldsymbol{v} \cdot \boldsymbol{w}^{\prime}-q^{\prime} \operatorname{div}(\boldsymbol{w})\right) \mathrm{d} x= \\
& \qquad \int_{\Omega_{f}}-\rho c_{p} S \nabla T \cdot \boldsymbol{w}^{\prime} \mathrm{d} x+\frac{\partial \mathfrak{J}}{\partial\left(\boldsymbol{v}^{\prime}, p^{\prime}\right)}\left(\boldsymbol{w}^{\prime}, q^{\prime}\right),
\end{aligned}
$$

and we recall our convention whereby the point $(0, \boldsymbol{v}(\Gamma), p(\Gamma), T(\Gamma), \boldsymbol{u}(\Gamma))$, where the partial derivatives of $J$ are evaluated, is omitted.

Remark 10. The existence and uniqueness of a solution $(\boldsymbol{w}, q, S, \boldsymbol{r})$ in $H^{1}\left(\Omega_{f}, \mathbb{R}^{d}\right) \times\left(L^{2}\left(\Omega_{f}\right) / \mathbb{R}\right) \times H^{1}(\Omega) \times$ $H^{1}\left(\Omega_{s}, \mathbb{R}^{d}\right)$ to the adjoint system (4.9) to (4.11) follows from the same considerations as in the case of the state system (4.2) to (4.4), except when it comes to the linearized Navier-Stokes equation (4.11). The latter is well-posed provided the Reynolds number is sufficiently small; see [30], Chap. IV about this point.

Note that, as expected, the cascade dependency $(\boldsymbol{v}, q) \rightarrow T \rightarrow \boldsymbol{u}$ in the state variables (the variable on the right of the arrow depends on that on the left) is reversed into $\boldsymbol{r} \rightarrow S \rightarrow(\boldsymbol{w}, q)$ for the adjoint variables, which reflects the fact that the adjoint system is formally the linearized transposition of the state problem.

Remark 11. Let us consider the case of a particular objective functional of the form

$$
J(\Gamma, \boldsymbol{v}(\Gamma), p(\Gamma), T(\Gamma), \boldsymbol{u}(\Gamma))=\int_{\Omega_{f}} j_{f}(\boldsymbol{v}(\Gamma), p(\Gamma), T(\Gamma)) \mathrm{d} x+\int_{\Omega_{s}} j_{s}(\boldsymbol{u}(\Gamma), T(\Gamma)) \mathrm{d} x
$$

where $j_{f}: \mathbb{R}_{\boldsymbol{v}}^{d} \times \mathbb{R}_{p} \times \mathbb{R}_{T} \rightarrow \mathbb{R}$ and $j_{s}: \mathbb{R}_{\boldsymbol{u}}^{d} \times \mathbb{R}_{T} \rightarrow \mathbb{R}$ are smooth and satisfy adequate growth conditions. The adjoint equations (4.9) to (4.11) rewrite respectively in strong form, for the elasticity system,

$$
\left\{\begin{array}{rlrl}
-\operatorname{div}(A e(\boldsymbol{r})) & =\frac{\partial j_{s}}{\partial \boldsymbol{u}} & \text { in } \Omega_{s} \\
\boldsymbol{r} & =0 & \text { on } \partial \Omega_{s}^{D} \\
A e(\boldsymbol{r}) \boldsymbol{n} & =0 & \text { on } \partial \Omega_{s}^{N} \cup \Gamma \\
17 &
\end{array}\right.
$$


for the thermal equation,

$$
\left\{\begin{aligned}
-\operatorname{div}\left(k_{s} \nabla S_{s}\right) & =\alpha \operatorname{div}(\boldsymbol{r})+\frac{\partial j_{s}}{\partial T_{s}} & \text { in } \Omega_{s} \\
-\operatorname{div}\left(k_{f} \nabla S_{f}\right)-\rho c_{p} \boldsymbol{v} \cdot \nabla S_{f} & =\frac{\partial j_{f}}{\partial T_{f}} & \text { in } \Omega_{f} \\
S & =0 & \text { on } \partial \Omega_{T}^{D} \\
k_{s} \frac{\partial S_{s}}{\partial n} & =0 & \text { on } \partial \Omega_{T}^{N} \cap \partial \Omega_{s} \\
k_{f} \frac{\partial S_{f}}{\partial n}+\rho c_{p}(\boldsymbol{v} \cdot \boldsymbol{n}) S_{f} & =0 & \text { on } \partial \Omega_{T}^{N} \cap \partial \Omega_{f} \\
S_{s} & =S_{f} & \text { on } \Gamma \\
-k_{s} \frac{\partial S_{s}}{\partial n} & =-k_{f} \frac{\partial S_{f}}{\partial n} & \text { on } \Gamma,
\end{aligned}\right.
$$

for the Navier-Stokes equations,

$$
\left\{\begin{aligned}
-\operatorname{div}\left(\sigma_{f}(\boldsymbol{w}, q)\right)+\rho\left(\nabla \boldsymbol{v}^{T} \boldsymbol{w}-\nabla \boldsymbol{w} \boldsymbol{v}\right) & =-\rho c_{p} S \nabla T_{f}+\frac{\partial j_{f}}{\partial \boldsymbol{v}} & & \text { in } \Omega_{f} \\
-\operatorname{div}(\boldsymbol{w}) & =\frac{\partial j_{f}}{\partial p} & & \text { in } \Omega_{f} \\
\boldsymbol{w} & =0 & & \text { on } \partial \Omega_{f}^{D} \\
\sigma_{f}(\boldsymbol{w}, q) \boldsymbol{n}+\rho(\boldsymbol{v} \cdot \boldsymbol{n}) \boldsymbol{w} & =0 & & \text { on } \partial \Omega_{f}^{N} \\
\boldsymbol{w} & =\boldsymbol{r} & & \text { on } \Gamma
\end{aligned}\right.
$$

Note the surprising fact in the linearized adjoint system (4.13) for Navier-Stokes equations that the interface condition for the velocity variable $\boldsymbol{w}$ is of Dirichlet type on $\Gamma$, while it was of Neumann type for the direct elasticity problem (2.3).

A very similar analysis to that of Section 3 yields the shape derivative of $J$ in the present physical context; see Appendix A for the proofs.

Proposition 4. Assume that the transported objective function $\mathfrak{J}$, defined by (4.8), has continuous partial derivatives at $(\boldsymbol{\theta}, \hat{\boldsymbol{v}}, \hat{p}, \hat{T}, \hat{\boldsymbol{u}})=\left(0, \boldsymbol{v}\left(\Gamma_{\boldsymbol{\theta}}\right), p\left(\Gamma_{\boldsymbol{\theta}}\right), T\left(\Gamma_{\boldsymbol{\theta}}\right), \boldsymbol{u}\left(\Gamma_{\boldsymbol{\theta}}\right)\right)$. Then the objective function $J$, defined by (4.7), is differentiable with respect to $\boldsymbol{\theta} \in W_{0}^{1, \infty}\left(\Omega, \mathbb{R}^{d}\right)$ and the derivative reads

$$
\begin{aligned}
\frac{\mathrm{d}}{\mathrm{d} \boldsymbol{\theta}}\left[J\left(\Gamma_{\boldsymbol{\theta}}, \boldsymbol{v}\left(\Gamma_{\boldsymbol{\theta}}\right), p\left(\Gamma_{\boldsymbol{\theta}}\right), T\left(\Gamma_{\boldsymbol{\theta}}\right), \boldsymbol{u}\left(\Gamma_{\boldsymbol{\theta}}\right)\right)\right](\boldsymbol{\theta}) & \\
= & \frac{\partial \mathfrak{J}}{\partial \boldsymbol{\theta}}(\boldsymbol{\theta})+\int_{\Omega_{f}}\left[\boldsymbol{w} \cdot \operatorname{div}\left(\boldsymbol{f}_{f} \otimes \boldsymbol{\theta}\right)-\left(\sigma_{f}(\boldsymbol{v}, p): \nabla \boldsymbol{w}+\rho \boldsymbol{w} \cdot \nabla \boldsymbol{v} \cdot \boldsymbol{v}\right) \operatorname{div}(\boldsymbol{\theta})\right] \mathrm{d} x \\
& \quad+\int_{\Omega_{f}}\left[\sigma_{f}(\boldsymbol{v}, p):(\nabla \boldsymbol{w} \nabla \boldsymbol{\theta})+\sigma_{f}(\boldsymbol{w}, q):(\nabla \boldsymbol{v} \nabla \boldsymbol{\theta})+\rho \boldsymbol{w} \cdot(\nabla \boldsymbol{v} \nabla \boldsymbol{\theta}) \cdot \boldsymbol{v}\right] \mathrm{d} x \\
& \quad-\int_{\Omega_{s}} \operatorname{div}(\boldsymbol{\theta})\left(k_{s} \nabla T \cdot \nabla S\right) \mathrm{d} x-\int_{\Omega_{f}} \operatorname{div}(\boldsymbol{\theta})\left(k_{f} \nabla T \cdot \nabla S+\rho c_{p}(\boldsymbol{v} \cdot \nabla T) S\right) \mathrm{d} x \\
+\int_{\Omega_{s}} k_{s}\left(\nabla \boldsymbol{\theta}+\nabla \boldsymbol{\theta}^{T}\right) \nabla T \cdot \nabla S \mathrm{~d} x+\int_{\Omega_{f}}\left[k_{f}\left(\nabla \boldsymbol{\theta}+\nabla \boldsymbol{\theta}^{T}\right) \nabla T \cdot \nabla S+\rho c_{p} \boldsymbol{v} \cdot\left(\nabla \boldsymbol{\theta}^{T} \nabla T\right) S\right] \mathrm{d} x & \quad+\int_{\Omega_{s}} \operatorname{div}\left(Q_{s} \boldsymbol{\theta}\right) S \mathrm{~d} x+\int_{\Omega_{f}} \operatorname{div}\left(Q_{f} \boldsymbol{\theta}\right) S \mathrm{~d} x \\
+ & \int_{\Omega_{s}}\left[-\operatorname{div}(\boldsymbol{\theta}) \sigma_{s}(\boldsymbol{u}, T): \nabla \boldsymbol{r}+\sigma_{s}(\boldsymbol{u}, T):(\nabla \boldsymbol{r} \nabla \boldsymbol{\theta})+A e(\boldsymbol{r}):(\nabla \boldsymbol{u} \nabla \boldsymbol{\theta})+\boldsymbol{r} \cdot \operatorname{div}\left(\boldsymbol{f}_{s} \otimes \boldsymbol{\theta}\right)\right] \mathrm{d} x,
\end{aligned}
$$

where $\boldsymbol{r}, S, \boldsymbol{w}, q$ are the adjoint states defined by (4.9) to (4.11). 
Proposition 5. If in addition the state and adjoint variables $\boldsymbol{v}, T_{s}, T_{f}, \boldsymbol{u}, \boldsymbol{w}, S, \boldsymbol{r}$ (resp. p, q) have $H^{2}$ (resp $\left.H^{1}\right)$ regularity in their domain of definition, and if the partial derivative $\frac{\partial \mathfrak{J}}{\partial \boldsymbol{\theta}}$ has a decomposition of the form (3.41), then (4.14) rewrites as an integral over the interface $\Gamma$ depending only on the normal component $\boldsymbol{\theta} \cdot \boldsymbol{n}$ of $\boldsymbol{\theta}$ :

$$
\begin{aligned}
& \frac{\mathrm{d}}{\mathrm{d} \boldsymbol{\theta}}\left[J\left(\Gamma_{\boldsymbol{\theta}}, \boldsymbol{v}\left(\Gamma_{\boldsymbol{\theta}}\right), p\left(\Gamma_{\boldsymbol{\theta}}\right), T\left(\Gamma_{\boldsymbol{\theta}}\right), \boldsymbol{u}\left(\Gamma_{\boldsymbol{\theta}}\right)\right)\right](\boldsymbol{\theta}) \\
& =\frac{\overline{\partial \mathfrak{J}}}{\partial \boldsymbol{\theta}}(\boldsymbol{\theta})+\int_{\Gamma}\left(\boldsymbol{f}_{f} \cdot \boldsymbol{w}-\sigma_{f}(\boldsymbol{v}, p): \nabla \boldsymbol{w}+\boldsymbol{n} \cdot \sigma_{f}(\boldsymbol{w}, q) \nabla \boldsymbol{v} \cdot \boldsymbol{n}+\boldsymbol{n} \cdot \sigma_{f}(\boldsymbol{v}, p) \nabla \boldsymbol{w} \cdot \boldsymbol{n}\right)(\boldsymbol{\theta} \cdot \boldsymbol{n}) \mathrm{d} s \\
& +\int_{\Gamma}\left(k_{s} \nabla T_{s} \cdot \nabla S_{s}-k_{f} \nabla T_{f} \cdot \nabla S_{f}+Q_{f} S-Q_{s} S_{s}-2 k_{s} \frac{\partial T_{s}}{\partial n} \frac{\partial S_{s}}{\partial n}+2 k_{f} \frac{\partial T_{f}}{\partial n} \frac{\partial S_{f}}{\partial n}\right)(\boldsymbol{\theta} \cdot \boldsymbol{n}) \mathrm{d} s \\
& \quad+\int_{\Gamma}\left(\sigma_{s}\left(\boldsymbol{u}, T_{s}\right): \nabla \boldsymbol{r}-\boldsymbol{f}_{s} \cdot \boldsymbol{r}-\boldsymbol{n} \cdot A e(\boldsymbol{r}) \nabla \boldsymbol{u} \cdot \boldsymbol{n}-\boldsymbol{n} \cdot \sigma_{s}\left(\boldsymbol{u}, T_{s}\right) \nabla \boldsymbol{r} \cdot \boldsymbol{n}\right)(\boldsymbol{\theta} \cdot \boldsymbol{n}) \mathrm{d} s,
\end{aligned}
$$

where $\frac{\overline{\partial \mathfrak{J}}}{\partial \boldsymbol{\theta}}$ denotes the part of $\frac{\partial \mathfrak{J}}{\partial \boldsymbol{\theta}}$ that depends only on the normal trace $\boldsymbol{\theta} \cdot \boldsymbol{n} ;$ viz. (3.42).

Remark 12. Formula (4.14) is a volume expression of the shape derivative, while formula (4.15) is a surface expression of the same derivative. Formula (4.15) can be simplified a little by using the following identities on $\Gamma$, which arise as consequences of the boundary conditions featured in (2.1) to (2.3):

$$
\begin{aligned}
\boldsymbol{n} \cdot \sigma_{f}(\boldsymbol{w}, q) \nabla \boldsymbol{v} \cdot \boldsymbol{n} & =\sigma_{f}(\boldsymbol{w}, q): \nabla \boldsymbol{v} \\
\boldsymbol{n} \cdot \sigma_{f}(\boldsymbol{v}, p) \nabla \boldsymbol{w} \cdot \boldsymbol{n}-\boldsymbol{n} \cdot \sigma_{s}\left(\boldsymbol{u}, T_{s}\right) \nabla \boldsymbol{r} \cdot \boldsymbol{n} & =\sigma_{f}(\boldsymbol{v}, p): \nabla \boldsymbol{w}-\sigma_{s}(\boldsymbol{v}, p): \nabla \boldsymbol{r} .
\end{aligned}
$$

The above equations (4.14) and (4.15) generalize more classical shape derivatives expressions for each of the physics considered individually: elastic, thermic, and fluid parts coincide with expressions stated in e.g. [6, 29] for the elasticity, [58] for pure thermoelasticity, [43] for the thermal conductivity terms, and [45, 16, 21] for the Navier Stokes equations. However some terms of formula (4.15) vanishing for particular instances of the objective function $J$ (for example, $A e(\boldsymbol{r}) \cdot \boldsymbol{n}=0$ for objective functions written as a volume integral depending only on $\boldsymbol{u}$ and not on its gradient), may have not been written in previous works.

\section{NumericAl REPRESENTATION OF SHAPES AND THEIR DEFORMATIONS}

One critical issue in the device of shape optimization algorithms is the difficulty in finding a numerical representation of shapes which lends itself to accurate mechanical analyses (carried out e.g. with the Finite Element method), but also allows to efficiently account for their deformations. For instance, it is well-known [46] that representing shapes with a Finite Element mesh, and realizing their deformations by 'pushing their vertices' in the direction of the shape gradient leads in very few iterations to nearly flat (or worse, invalid) mesh elements. To overcome this difficulty, multiple numerical methods have been thought of that avoid representing the shape explicitly at each stage of the optimization process (at the cost of an approximate resolution of the mechanical problem at stake); let us mention, among others, level set methods $[5,6,41,50,56]$, phase field methods [10,53], etc. Not to mention others methods which are based on variable density parametrization (like SIMP [8]) and do not explicitly represent the shape's boundary. In the present article, we use the level set based mesh evolution method introduced in our previous work [4]. The main idea is to combine two numerical representations of the partition of the total computational domain $\Omega$ into the fluid and solid phases $\Omega_{f}$ and $\Omega_{s}$ (see Figure 3):

- On the one hand, $\Omega_{f}$ and $\Omega_{s}$ are implicitly described, using the level set method: $\Omega_{f}$ (resp. $\Omega_{s}$ ) is seen as the negative (resp. positive) subdomain of a function $\phi: \Omega \rightarrow \mathbb{R}$,

- On the other hand, a computational mesh of $\Omega$ is available, in which $\Omega_{f}$ and $\Omega_{s}$ are explicitly discretized.

We then consistently alternate between both descriptions of the partition $\Omega=\Omega_{f} \cup \Omega_{s}$ depending on the requirements of the ongoing operation: Finite Element resolutions are typically carried out using the meshed description, while the motion of the phases is tracked using the level set method.

In this section, we outline the main features of this method in the case of two space dimensions; note however that there is no conceptual difficulty in extending this process to the three-dimensional case (even 


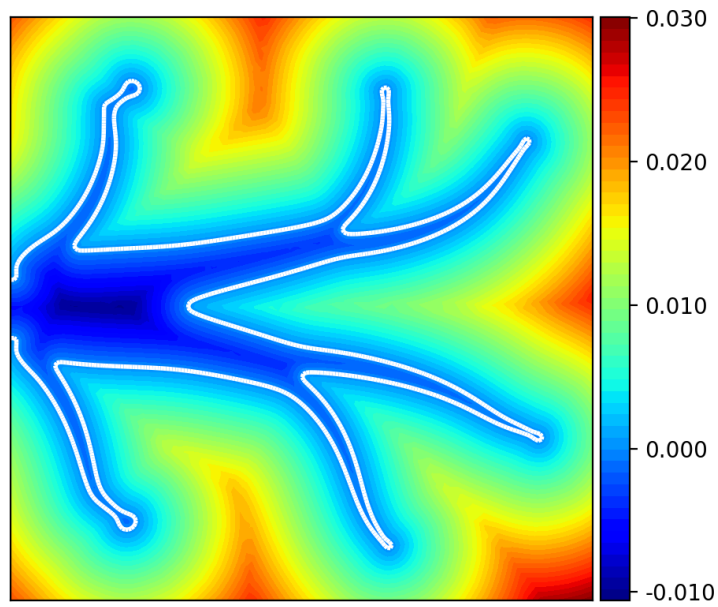

(A) Level set function $\phi$

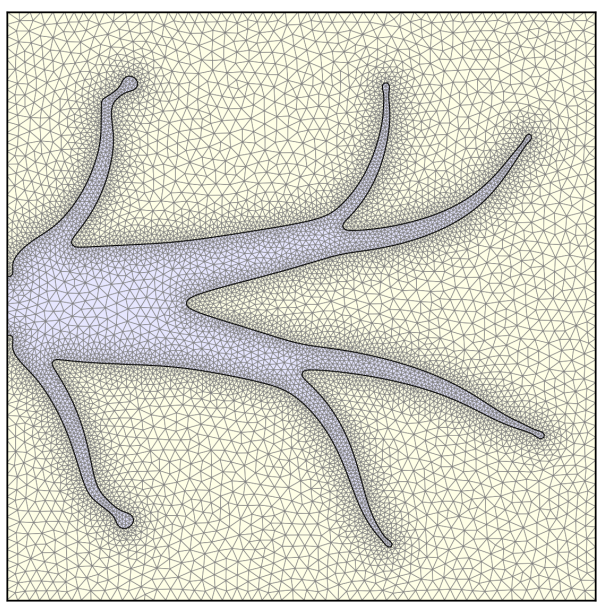

(B) Computational mesh

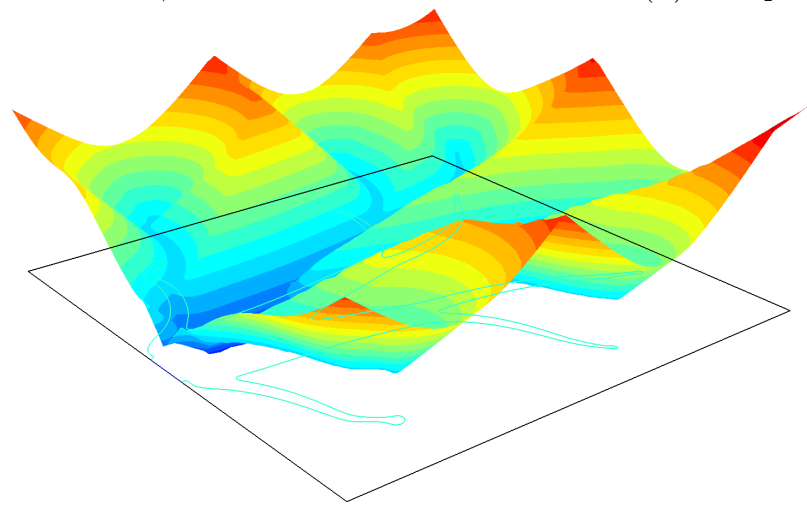

(C) Level set function $\phi(3 \mathrm{D}$ plot)

FiguRE 3. Representation of a two-phase partition $\Omega=\Omega_{f} \cup \Omega_{s}$ by means of a level set function on the computational mesh. The fluid-solid interface $\Gamma$ (the black line on picture (b)) delimits the boundary between the explicitly meshed subdomains $\Omega_{f}$ and $\Omega_{s}$.

if the involved algorithms are then much more tedious to implement); see [4].

\subsection{Two complementary numerical representations of shapes.}

\subsubsection{An implicit description via the level set method.}

The level set method was pioneered by S. Osher and J. Sethian in [42], then introduced in the shape optimization context in $[5,6,41,50,56]$. In our situation, it amounts to representing the fluid phase $\Omega_{f}$ (resp. the solid phase $\Omega_{s}$ ) as the negative (resp. positive) subdomain of a scalar 'level set' function $\phi: \Omega \rightarrow \mathbb{R}$, defined on the total computational domain $\Omega$ :

$$
\begin{cases}\phi(x)<0 & \text { if } x \in \Omega_{f}, \\ \phi(x)=0 & \text { if } x \in \Gamma \\ \phi(x)>0 & \text { if } x \in \Omega_{s} .\end{cases}
$$

The main asset of this description is that if $\Omega_{f} \equiv \Omega_{f}(t)$ and $\Omega_{s} \equiv \Omega_{s}(t)$ are evolving in time according to a (vector) velocity field $\boldsymbol{\theta}(t, x)$, the motion reformulates in terms of an associated level set function $\phi(t, \cdot)$ 
(i.e. (5.1) holds at every time $t>0$ ) as an advection equation:

$$
\frac{\partial \phi}{\partial t}(t, x)+\boldsymbol{\theta}(t, x) \cdot \nabla \phi(t, x)=0, t>0, x \in \Omega
$$

When the velocity field $\boldsymbol{\theta}(t, x)$ is consistently normal to the interface $\Gamma(t)$, i.e. $\boldsymbol{\theta}(t, x)=v(t, x) \boldsymbol{n}$ on $\Gamma$ for some scalar function $v(t, x),(5.2)$ rewrites as the well-known Hamilton-Jacobi equation:

$$
\frac{\partial \phi}{\partial t}(t, x)+V(t, x)|\nabla \phi(t, x)|=0, t>0, x \in \Omega .
$$

In our numerical setting, $\Omega$ is equipped with a simplicial mesh $\mathcal{T}$, and the level set function $\phi$ is represented as a $\mathbb{P}_{1}$ Lagrange Finite Element function on $\mathcal{T}$ (its values are stored at the vertices of $\mathcal{T}$ ). For convenience, we solve the evolution equation (5.2) on the unstructured mesh $\mathcal{T}$ by using an algorithm based on the method of characteristics. This method is issued from the previous work [13] and shares common features with the semi-Lagrangian method of J. Strain [52].

\subsubsection{A meshed description of the phases $\Omega_{s}$ and $\Omega_{f}$.}

An alternative way to represent a partition of the computational domain $\Omega$ into a fluid and a solid phase $\Omega_{f}$ and $\Omega_{s}$ is to consider a simplicial mesh $\mathcal{T}_{\Gamma}$ of the whole domain $\Omega$ (the subscript ${ }_{\Gamma}$ emphasizes the fact that it depends on the partition $\Omega=\Omega_{f} \cup \Omega_{s}$ ), that is, a discretization

$$
\bar{\Omega}=\bigcup_{n=1}^{N} T_{n}
$$

of $\bar{\Omega}$ into a collection of non overlapping (closed) triangles $T_{n}, n=1, \ldots, N$. We additionally impose that $\mathcal{T}_{\Gamma}$ fulfill the following properties:

- The mesh $\mathcal{T}_{\Gamma}$ is globally conforming in the sense of Finite Elements, i.e. the intersection between any two triangles $T_{i}$ and $T_{j}$ is either empty, or reduced to a vertex, or reduced to an edge of the mesh;

- The shape $\Omega_{f}$ and its complement $\Omega_{s}$ are explicitly discretized in $\mathcal{T}_{\Gamma}$; to be precise, $\mathcal{T}_{\Gamma}=\mathcal{T}_{f} \cup \mathcal{T}_{s}$ is the disjoint reunion of two triangular meshes $\mathcal{T}_{f}$ and $\mathcal{T}_{s}$ for $\Omega_{f}$ and $\Omega_{s}$ respectively.

This representation makes each phase $\Omega_{f}, \Omega_{s}$ readily available for mechanical computations. This is quite convenient when it comes to solving (2.1) to (2.3) and to computing the shape derivative (4.15) of the optimized functional. Moreover, it lends itself to the use of external commercial solvers since the numerical resolution of the state equations (2.1), (2.2) and (2.3) does not require any (intrusive) approximation or penalization such as the Ersatz material method in the context of linear elasticity.

\subsection{Switching between both representations of shapes.}

The success of combining an implicit and a meshed representation of the partition $\Omega=\Omega_{f} \cup \Omega_{s}$ relies on two algorithms for passing from one to the other, as we outline in this section.

\subsubsection{Generating a level set function associated to a domain.}

Let $\Omega$ be partitioned into a fluid and a solid phase $\Omega_{f}$ and $\Omega_{s}$, in such a way that $\Omega$ is equipped with a triangular mesh $\mathcal{T}_{\Gamma}$ in which $\Omega_{f}$ and $\Omega_{s}$ are explicitly discretized. A level set function satisfying (5.1) may be conveniently generated at the vertices of $\mathcal{T}_{\Gamma}$ as the signed distance function $d_{\Omega_{f}}$ to $\Omega_{f}$ :

$$
\forall x \in \Omega, \quad d_{\Omega_{f}}(x)=\left\{\begin{array}{cl}
-d(x, \Gamma) & \text { if } x \in \Omega_{f}, \\
0 & \text { if } x \in \Gamma, \\
d(x, \Gamma) & \text { if } x \in \Omega_{s},
\end{array}\right.
$$

where $d(x, \Gamma)=\min _{p \in \Gamma}|x-p|$ is the usual Euclidean distance from a point $x \in \Omega$ to $\Gamma$. 
Numerous algorithms are available to carry out this operation, such as the celebrated Fast Marching Method (see [49], or [35] for a version on simplicial meshes), or the Fast Sweeping method [61]. Here we rely on an algorithm from the previous work [20]. Notice that this part does not require $\Omega_{f}$ and $\Omega_{s}$ to be explicitly discretized in the computational mesh of $\Omega$.

\subsubsection{From a level set function to a mesh.}

The situation is the following: the fixed computational domain $\Omega$ is equipped with a triangular mesh $\mathcal{T}$. A $\mathbb{P}_{1}$ Lagrange function $\phi$ is defined at the vertices of $\mathcal{T}$, whose negative (resp. positive) subdomain is the fluid phase of interest $\Omega_{f}$ (resp. the solid phase $\Omega_{s}$ ); viz. (5.1). We aim at constructing a new mesh $\mathcal{T}_{\Gamma}$ of $\Omega$ in such a way that $\Omega_{f}$ and $\Omega_{s}$ (thus $\Gamma$ ) are explicitly discretized in $\mathcal{T}_{\Gamma}$. This is achieved owing to the methodology introduced in [18], which proceeds within two steps, as illustrated on Figure 5:

Step 1: Rough discretization of $\Gamma$ in the mesh of $\Omega$ :

During this first step, the triangles $T \in \mathcal{T}$ crossed by the interface $\Gamma$ are subdivided in such a way that $\Omega_{f}, \Omega_{s}$ and $\Gamma$ explicitly appear in the resulting mesh $\widetilde{\mathcal{T}}$.

To achieve this, every triangle $T \in \mathcal{T}$ is inspected. Denoting its vertices by $a_{0}, a_{1}$ and $a_{2}$ (in 2-d), $T$ is crossed by $\Gamma$ if and only if the values $\phi\left(a_{0}\right), \phi\left(a_{1}\right)$ and $\phi\left(a_{2}\right)$ are not all three of the same sign. In such a case, $\phi$ being linear inside $T$, the portion of segment $\Gamma \cap T=\{x \in T, \phi(x)=0\}$ is calculated from these three values. The triangle $T$ is then split into two or three triangles according to a predefined pattern, in such a way that $\Gamma \cap T$ explicitly appears in the subdivision.

This simple step results in a new, conforming mesh $\widetilde{\mathcal{T}}$ of $\Omega$ in which $\Omega_{f}$ and $\Omega_{s}$ are explicitly discretized. Unfortunately, $\widetilde{\mathcal{T}}$ has poor quality when it comes to Finite Element calculations: it contains very ill-shaped, nearly flat elements which may jeopardize the accuracy of mechanical calculations; see Figure 5 (b).

\section{Step 2: Local remeshing of $\widetilde{\mathcal{T}}$ into a good-quality mesh $\mathcal{T}_{\Gamma}$}

Starting from the low-quality mesh $\widetilde{\mathcal{T}}$, local remeshing operations are iteratively performed to result in a good quality mesh of $\Omega$, in which $\Omega_{f}$ and $\Omega_{s}$ are still explicitly discretized. These four operations are quite classical in meshing practice; we refer to Figure 4 for an illustration and to e.g. [28] for more details:

- Edge split: Split a 'too long' edge in the mesh;

- Vertex collapse: Merge the two ends of a 'too short' edge in the mesh;

- Edge swap: Flip the common edge between two adjacent triangles to the alternative configuration;

- Vertex relocation: Slightly move one node in the mesh by keeping all the other ones fixed, and constant connectivities.

These operations are iteratively carried out as long as they improve the overall quality of the mesh. Let us emphasize that their application has to be be carefully monitored, since they may entail invalid configurations, showing e.g. overlapping elements. Moreover, additional controls have to be performed when they are applied to configurations near the boundaries $\Gamma$ or $\partial \Omega$, so that their geometric representation is not deteriorated. Eventually, with these operators, it is easy to adapt the density of triangles in a desired region of space, so that, for instance, the resulting mesh $\mathcal{T}_{\Gamma}$ has smaller elements near the interface $\Gamma$.

\subsection{The shape optimization algorithm.}

We now summarize the main steps of one typical iteration of our shape optimization strategy for minimizing a function $J\left(\Gamma, u_{f}(\Gamma), u_{s}(\Gamma)\right)$. At the beginning of iteration $n$, it is assumed that a mesh $\mathcal{T}_{\Gamma^{n}}$ of $\Omega$ is available in which the phases $\Omega_{f}^{n}, \Omega_{s}^{n}$ and the interface $\Gamma^{n}$ are explicitly discretized in the sense of Section 5.1.2. The following operations are then performed, and result in a new mesh $\mathcal{T}_{\Gamma^{n+1}}$ of $\Omega$ with an (updated) explicitly discretized partition $\Omega=\Omega_{f}^{n+1} \cup \Omega_{s}^{n+1}$; see Figure 5:

(1) Generate the signed distance function $\phi^{n}=d_{\Omega_{f}^{n}}$ to $\Omega_{f}^{n}$ on the whole mesh $\mathcal{T}_{\Gamma^{n}}$ of $\Omega$.

(2) Solve the state equations (2.1), (2.2) or (2.3) on the fluid mesh $\mathcal{T}_{f}^{n}$, the total mesh $\mathcal{T}_{\Gamma^{n}}$ and the elastic mesh $\mathcal{T}_{s}^{n}$ respectively. 

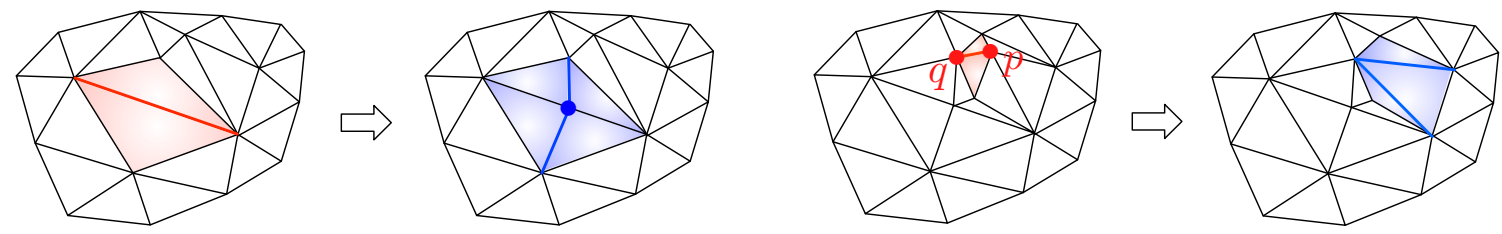

(A)

(B)
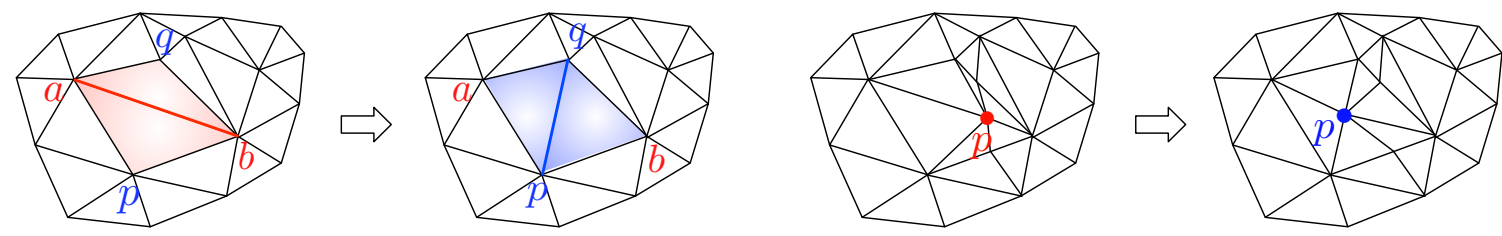

(c)

(D)

FiguRE 4. Local remeshing operations: (a) split of the red edge by insertion of a new vertex; (b) collapse of the 'small' edge $p q$; (c) swap of the edge $a b$ for the alternate configuration $p q$; (d) relocation of point $p$ to improve the quality of the local configuration.

(3) Find a descent direction by solving the identification (or extension-regularization) problem (3.15) for a discrete scalar product $a$. Doing so yields an extension and regularization $\boldsymbol{\theta}^{n}$ of the shape derivative of $J$ on the whole computational mesh $\mathcal{T}_{\Gamma^{n}}$.

(4) Solve the advection equation (5.2) on $\mathcal{T}_{\Gamma^{n}}$ for a small time step using $\phi^{n}$ as the initial state. This yields a $\mathbb{P}_{1}$ level set function $\widehat{\phi^{n+1}}$ on the mesh $\mathcal{T}_{\Gamma^{n}}$ for the deformed configuration $\Gamma^{n+1}$.

(5) Adapt the mesh $\mathcal{T}_{\Gamma^{n}}$ to the level Set function $\widetilde{\phi^{n+1}}$ by using the methodology of Section 5.2.2. A new mesh $\mathcal{T}_{\Gamma^{n+1}}$ of $\Omega$ is obtained in which $\Gamma^{n+1}$ is explicitly described.

\section{NumERICAL EXAMPLES}

In this section, we present and discuss several numerical examples in the three-physic context detailed in Section 2, or in simplified instances of it. We demonstrate on these multiphysics examples how the previous ideas can be effectively implemented in order to address a wide range of topology optimization problems.

\subsection{Details about the numerical implementation.}

Our numerical implementation relies on the open-source FreeFem++ environment for the resolution of Finite Element problems [31] (see [7] for its use in the context of structural optimization and [21] about its use in the context of fluid flow optimization).

The Navier-Stokes system (4.2) is solved by using a mixed formulation, where the space $V_{\boldsymbol{v}, p}$ is discretized with $\mathbb{P}_{1}$-bubble $\times \mathbb{P}_{1}$ elements. The equations (2.2) and (2.3) for the temperature $T$ and the elastic displacement $\boldsymbol{u}$ are solved with $\mathbb{P}_{1}$ finite elements. In all the considered examples, the Reynolds number and the velocity $\boldsymbol{v}$ of the fluid are sufficiently small so that the convergence and stability of our numerical schemes are guaranteed without the need for more complex numerical strategies, e.g. upwinding methods. Note that, when solving the fluid-structure interaction problem (2.3), a numerical estimate of the normal stress $\sigma_{f}(\boldsymbol{v}, p) \boldsymbol{n}$ is used as a boundary load in the discretization of the variational formulation (4.4). Naturally, a mixed formulation analogous to (3.51) could be implemented for the triplet $(\boldsymbol{v}, p, \boldsymbol{u})$, which would avoid computing normal derivatives at the boundary.

As far as the calculation of a descent direction for the considered objective function $J$ is concerned, the identification problem (3.15) (also called extension and regularization of the interface velocity) is solved using FreeFem++, based on the surface expression (4.15) for the shape derivative for the first two examples Sections 6.2 and 6.3 and the volumetric (4.14) for the third example Section 6.4. 


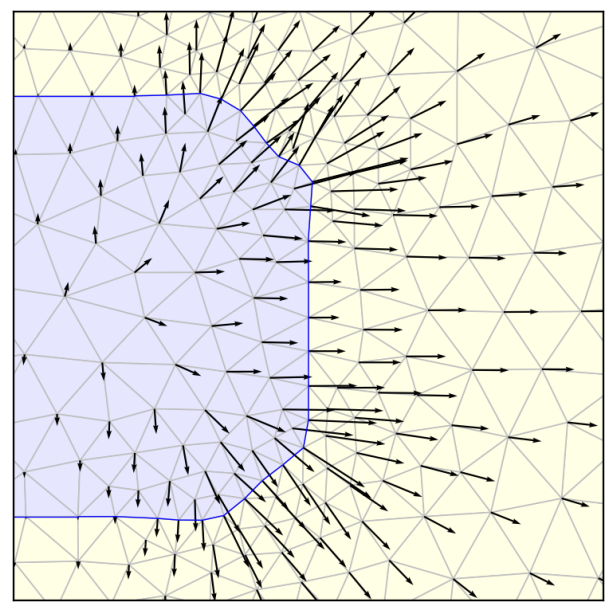

(A)

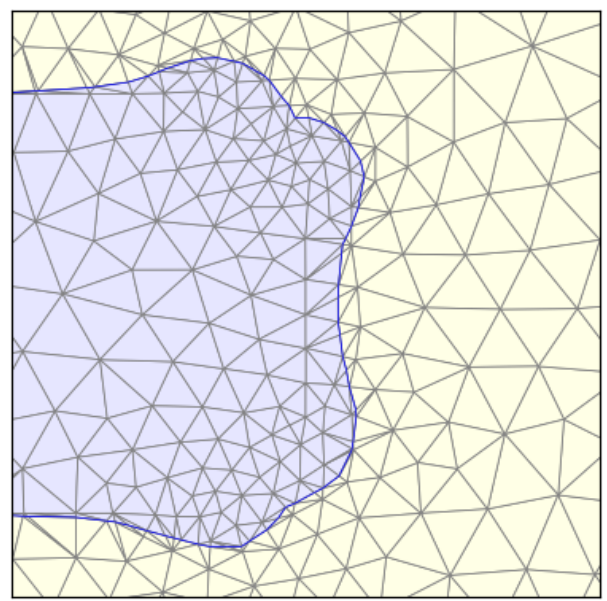

(c)

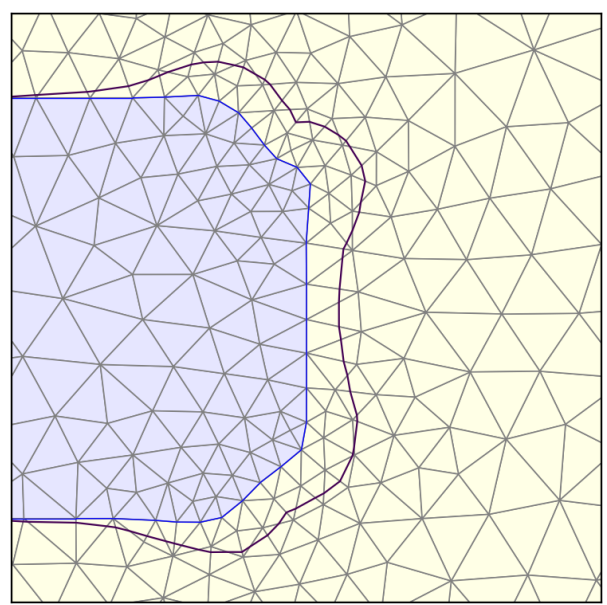

(B)

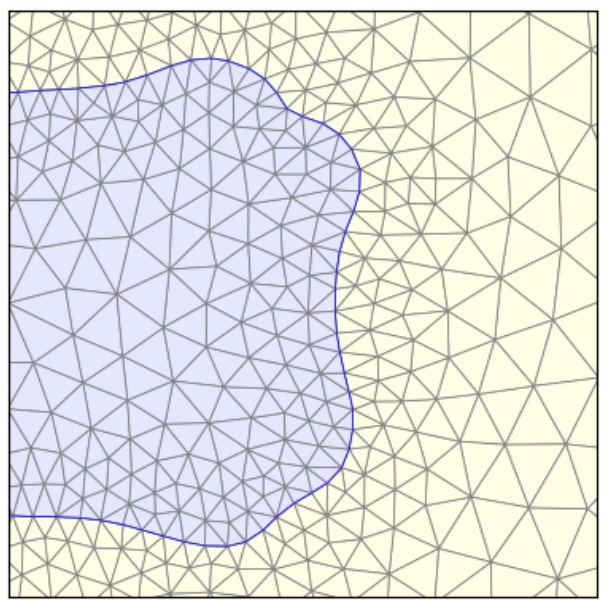

(D)

Figure 5. (a) A vector field $\boldsymbol{\theta}$ is defined at the vertices of the computational mesh $\mathcal{T}$ in which $\Omega_{s}$ and $\Omega_{f}$ are explicitly discretized in $\mathcal{T}$; (b) a level set function $\phi$ associated with the partition $\Omega_{s} \cup \Omega_{f}$ is advected on $\mathcal{T}$; (c) A poor quality mesh $\tilde{\mathcal{T}}$ is obtained by splitting $\mathcal{T}$ according to the updated level set function ; (d) $\tilde{\mathcal{T}}$ is iteratively remeshed into a new mesh $\mathcal{T}_{\Gamma}$ of sufficient quality for Finite Element analysis.

The open-source library mmg is used when it comes to the isosurface discretization and quality-oriented remeshing operations outlined in Section 5.2.2; see [18, 17]. As far as the initial computation of the signed distance function to a meshed domain is concerned (see Section 5.2.1), we rely on the open-source algorithm mshdist; see [20].

In all our examples, the considered shape and topology optimization problem features an equality constraint, e.g. on the volume of one of the two phases $\Omega_{f}, \Omega_{s}$. Such a constraint is progressively enforced using an analytical guess for the Lagrange multiplier with a method very close to that in [7].

\subsection{A steady-state fluid-structure interaction problem.}

In this first example, a fluid is flowing through a pipe, where it is pushing on a vertical beam of solid clamped at its bottom; see Figure 6 for the details of the test case. We neglect the thermal effects (namely, (2.2) 


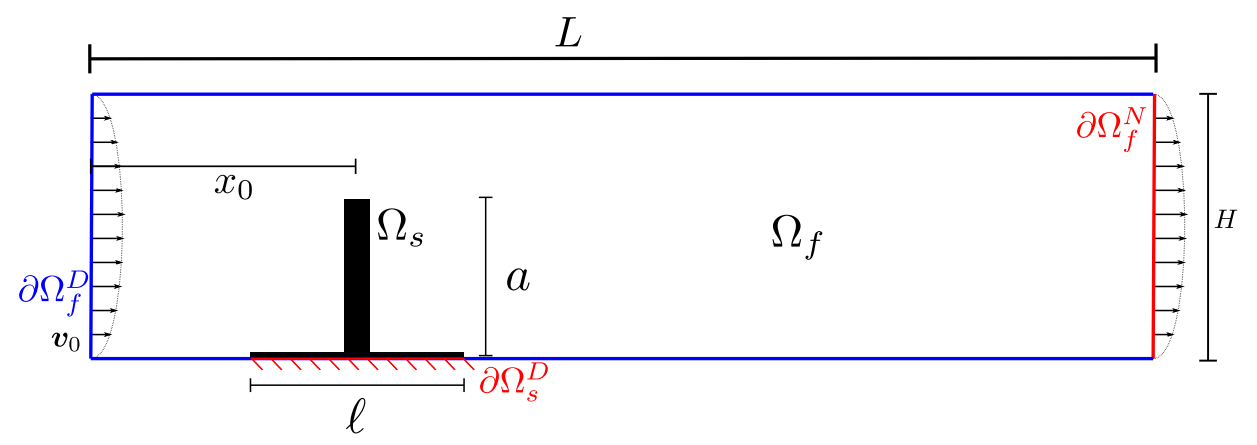

Figure 6. Physical setting of the fluid-structure optimization problem of Section 6.2. During optimization, the black domain cannot be reduced but only enlarged by adding reinforcements.

\begin{tabular}{cccccccc}
\hline$L$ & $H$ & $\ell$ & $a$ & $\nu$ & $\rho$ & $\lambda$ & $\mu$ \\
\hline \hline 2 & 0.5 & 0.4 & 0.3 & 0.005 & 1 & 0.00529 & 0.0476 \\
\hline
\end{tabular}

TABLE 1. Numerical values of the physical parameters for the fluid-structure problem of Section 6.2

is ignored), so that (2.3) boils down to a standard linear elasticity system with the forcing induced by the fluid. Our aim is to minimize the compliance of the solid phase $\Omega_{s}$, that is:

$$
J(\Gamma, \boldsymbol{u}(\Gamma))=\int_{\Omega_{s}} A e(\boldsymbol{u}): e(\boldsymbol{u}) \mathrm{d} x .
$$

This example was previously considered by Yoon [60] with a different density-based (SIMP) method. In this setting, the formulas (3.47) and (3.48) needed for the shape derivative of $J$ and the definition of the adjoint systems read:

$$
\begin{gathered}
\frac{\partial \mathfrak{J}}{\partial \boldsymbol{\theta}}(\boldsymbol{\theta})=\int_{\Omega_{s}}(A e(\boldsymbol{u}): e(\boldsymbol{u}) \operatorname{div}(\boldsymbol{\theta})-2 A e(\boldsymbol{u}):(\nabla \boldsymbol{u} \nabla \boldsymbol{\theta})) \mathrm{d} x \\
\frac{\overline{\partial \mathfrak{J}}}{\partial \boldsymbol{\theta}}(\boldsymbol{\theta})=\int_{\Gamma}(-A e(\boldsymbol{u}): e(\boldsymbol{u})+2 \boldsymbol{n} \cdot(A e(\boldsymbol{u})) \nabla \boldsymbol{u} \cdot \boldsymbol{n})(\boldsymbol{\theta} \cdot \boldsymbol{n}) \mathrm{d} s \\
\frac{\partial \mathfrak{J}}{\partial \hat{\boldsymbol{u}}}\left(\boldsymbol{r}^{\prime}\right)=\int_{\Omega_{s}} 2 A e(\boldsymbol{u}): e\left(\boldsymbol{r}^{\prime}\right) \mathrm{d} x .
\end{gathered}
$$

The numerical values of the considered physical parameters are supplied in Table 1 . The velocity profile $\boldsymbol{v}_{0}$ imposed at the entrance of the pipe is parabolic, with maximum amplitude $v_{\max }=1$, and value 0 at the upper and lower walls. The Reynolds number computed from the height of the vertical beam is equal to $\operatorname{Re}=\frac{\rho h v_{\max }}{\nu}=60$. The elastic displacement is set to 0 on a horizontal segment of length $\ell$ supporting the beam.

The functional $J$ is minimized using the shape optimization algorithm of Section 5.3, under the constraint that the volume of the solid phase represent a fraction $\eta_{s}=\left|\Omega_{s}\right| /|\Omega|=0.025$ of that of the total domain. The initial and optimized shapes, and the corresponding fluid velocity fields $\boldsymbol{v}$ are displayed on Figure 8 . The evolution of the objective function and of the volume fraction are reported on Figure 7 . Note that in the first part of the optimization, $J$ increases sometimes substantially due to the fact that the volume constraint is not yet satisfied, or due to sudden discontinuities at topological changes. On Figure 9, several intermediate shapes are displayed, which further illustrates a key feature of our numerical method: an explicitly meshed, black and white description of the shape is available during the whole optimization process. Our final design 


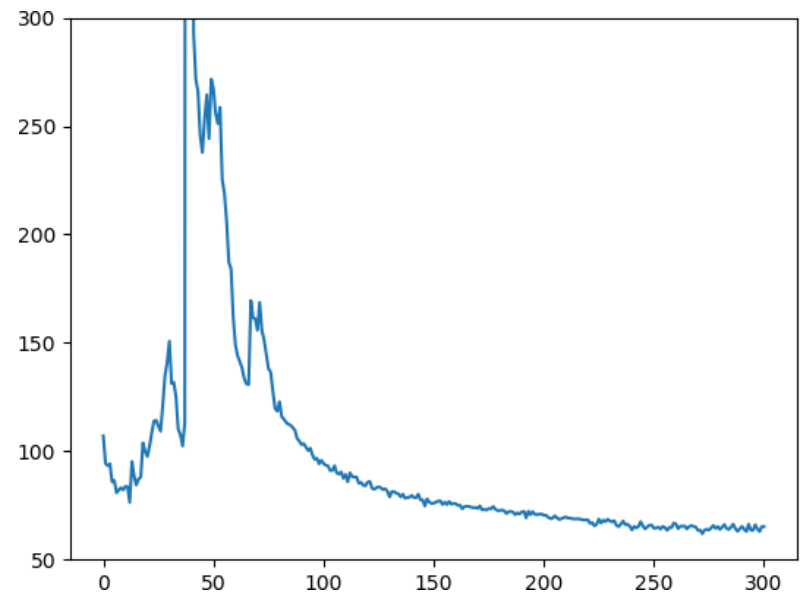

(A) Objective function $J$

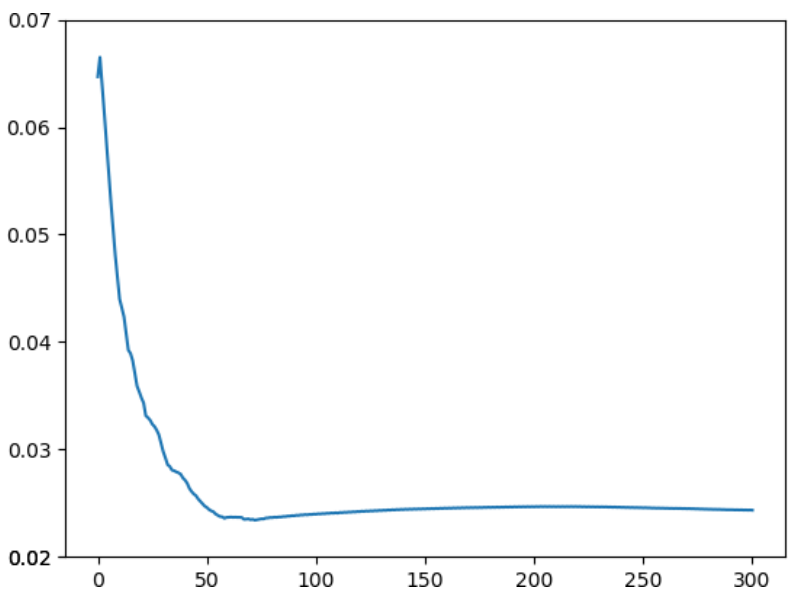

(B) Volume fraction

FiguRE 7. Convergence histories for the fluid-structure optimization problem of Section 6.2.
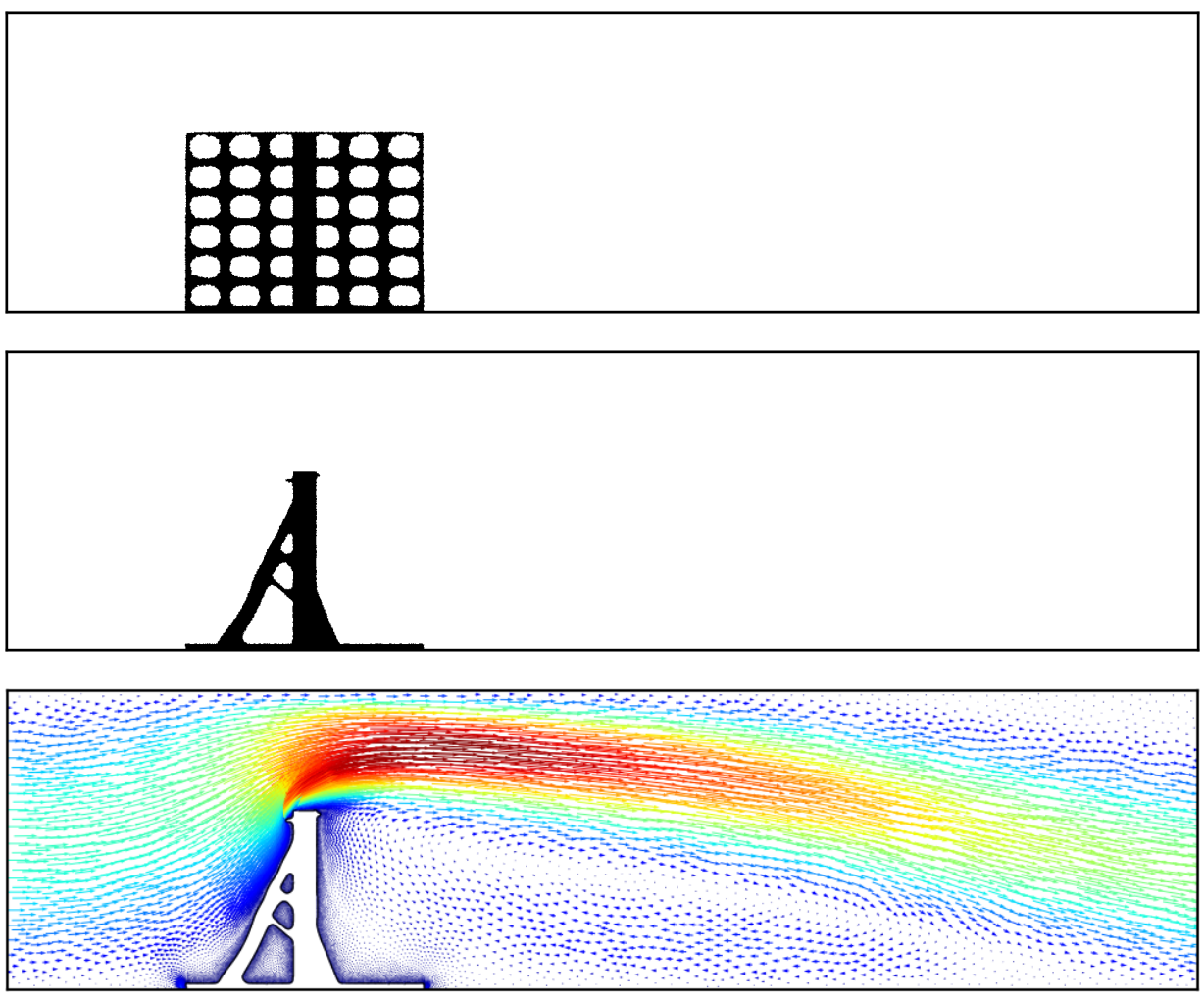

Figure 8. Top: initialization. Middle: optimized shape in the fluid-structure example of Section 6.2 (the solid phase is depicted in black); bottom: velocity field $\boldsymbol{v}$ associated to the optimized shape.

is different from that in [60] because our Reynolds number is much bigger and the location of the beam is different.

Finally, Figure 10 shows parts of the initial and the final mesh. We point out that we used a feature of the library $\mathrm{mmg}$ to selectively enforce a fine mesh resolution on the discrete interface $\Gamma$, while allowing larger triangles far from this boundary in order to save computational effort. Note in particular the small 

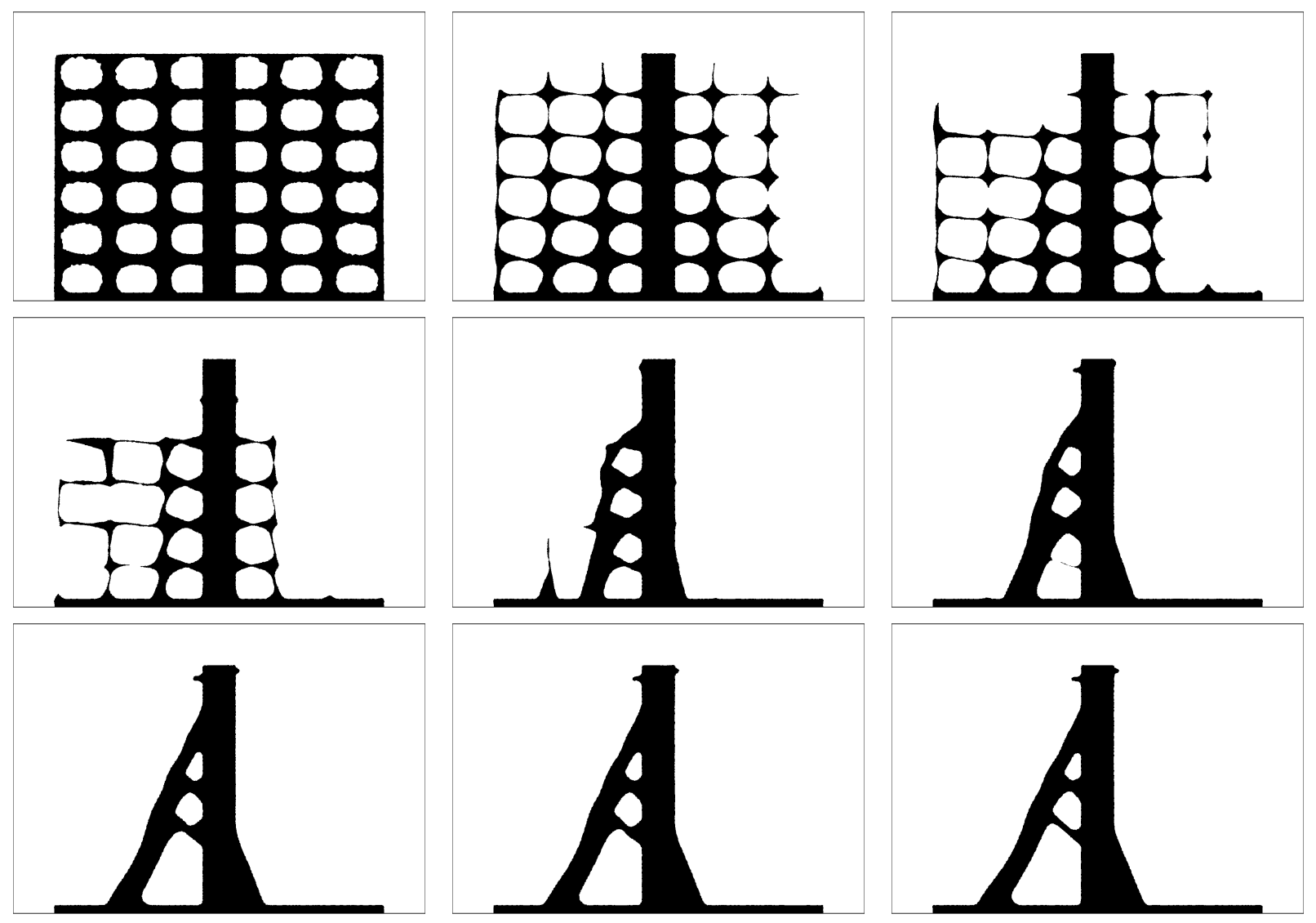

FigURE 9. Optimization history for the steady state fluid-structure optimization problem of Section 6.2: iterations 1, 15, 30, 47, 75, 110, 220, 236 and 300

solid bumps near the top of the vertical beam which appear at the end of the optimization and which we checked led indeed to a better design: the objective function value for the optimized design is $J=65.2$ versus $J=74.3$ for the same design without the two inclusions, for a negligible variation of the volume fraction. The mesh resolutions evolve from approximatively 28,000 vertices near the first iterations to 7,000 at convergence. The optimization ran in approximately one hour on a laptop equipped with Intel(R) Core(TM) i7-4702MQ @ 2.20 GHz. Note that no optimization of the implementation was performed for reducing the total computational time, e.g. with the use of preconditionners or parallelism when solving finite element problems that represent most of the computational effort for these 2D examples.

\subsection{Convective heat transfer.}

Our second example involves only a coupling of the flow and heat equations (2.1) and (2.2), i.e. the elastic behavior of the complement $\Omega_{s}$ of the optimized fluid phase $\Omega_{f}$ is not taken into account (equation (2.3) is ignored). This test case features a cavity where a fluid is entering with a parabolic profile (with maximal velocity $v_{\max }=1$ ) and an inlet temperature $T_{\mathrm{in}}$. The setting is similar to that in [38] (see Figure 11), although we use different parameters values; namely, a higher Reynold number $\left(\mathrm{Re}=\frac{\rho L v_{\max }}{5 \nu}=40\right.$ while $\mathrm{Re}=3$ in [38]) and different prescribed temperatures $T_{\text {up }}$ and $T_{\text {low }}$ for the lower and upper walls respectively (the other regions of the boundary of the cavity are insulated from the outside, i.e. zero normal fluxes boundary conditions hold for the temperature). 

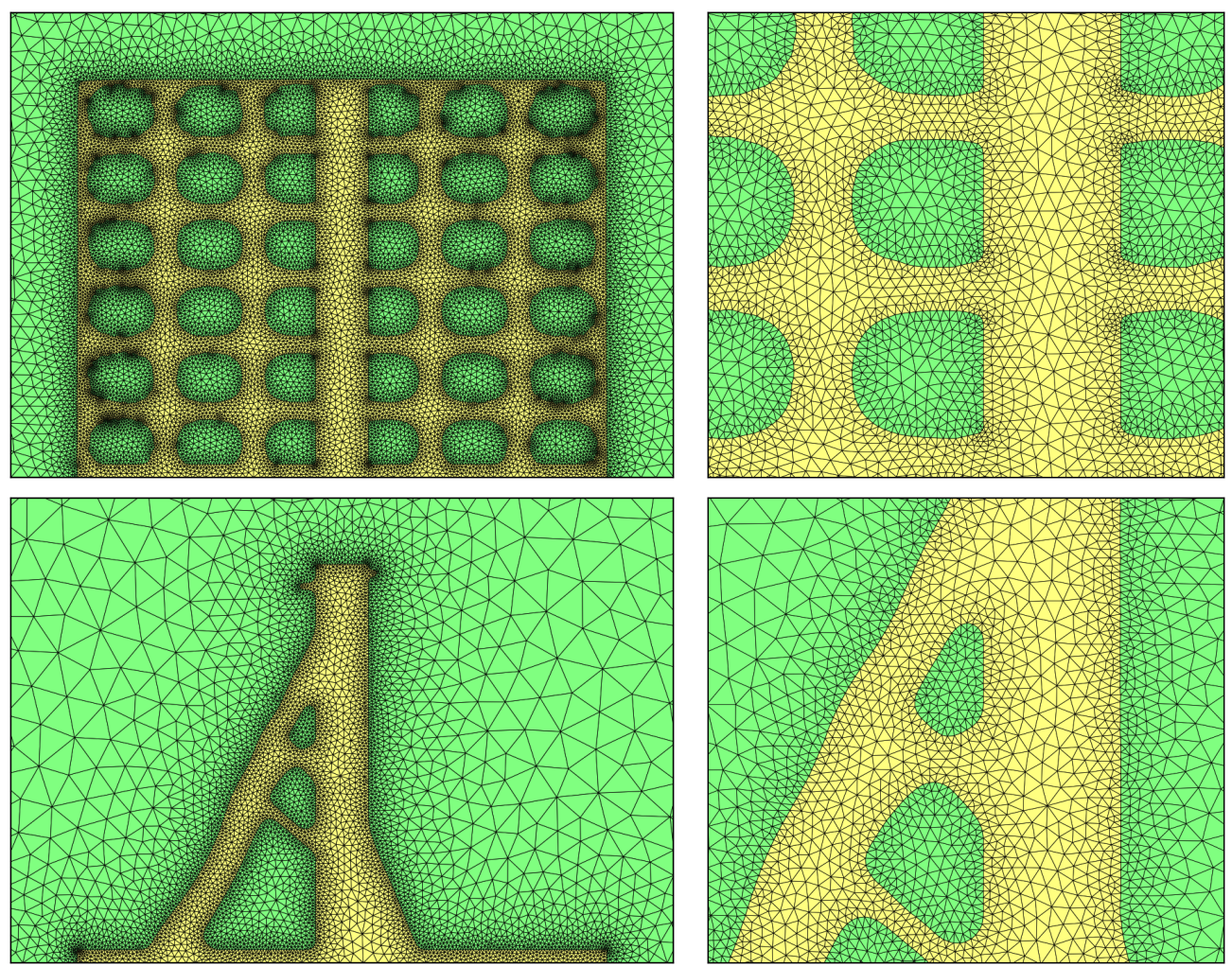

Figure 10. Zooms on the meshes of the initial and final shapes in the fluid-structure interaction problem of Section 6.2.

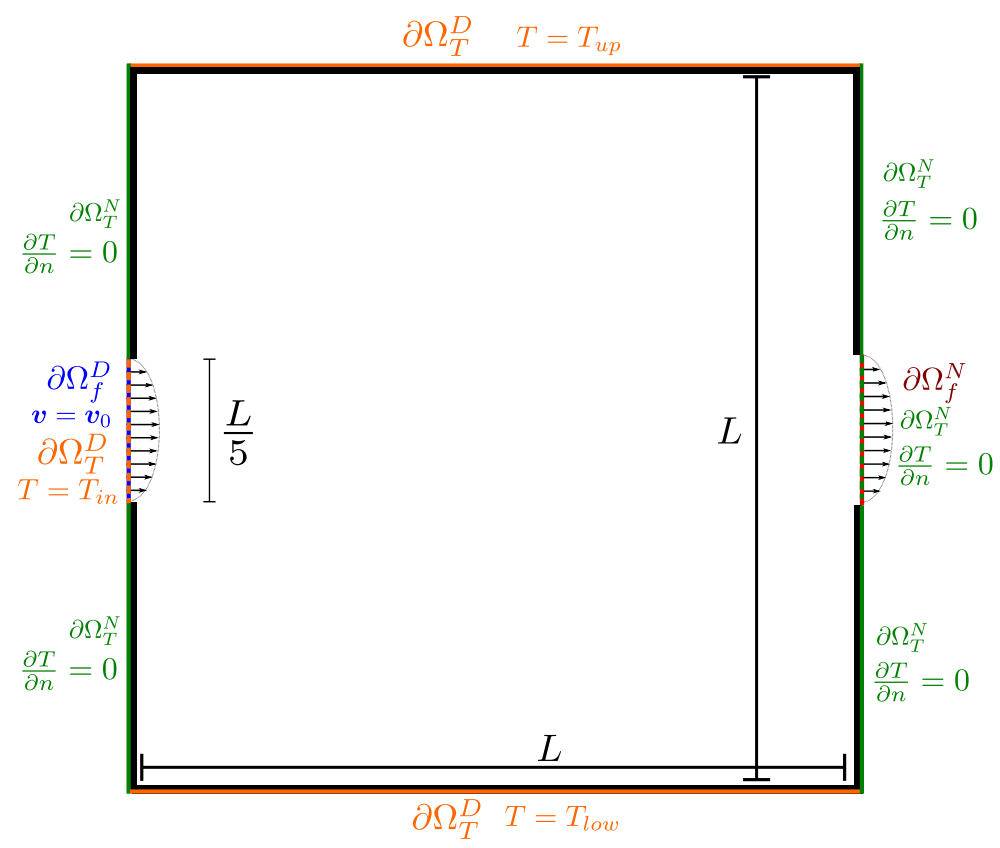

Figure 11. Setting of the convective heat transfer test case of Section 6.3. The black layers at the walls stand for solid, non optimizable boundaries. 


\begin{tabular}{ccccccccccccc}
\hline$L$ & $\rho$ & $c_{p}$ & $\nu$ & $v_{\max }$ & $k_{s}$ & $k_{f}$ & $\omega$ & $T_{\text {in }}$ & $T_{\text {low }}$ & $T_{\text {up }}$ (Ex. 1) & $T_{\text {up }}$ (Ex. 2) \\
\hline \hline 0.1 & 10 & 100 & 0.005 & 1 & 10 & 1 & 0.4 & 0 & 10 & -5 & 10 \\
\hline
\end{tabular}

TABLE 2. Numerical values of the physical parameters in the convective heat transfer problem of Section 6.3.

Our aim is to achieve a trade-off between the minimization of the viscous energy dissipated by the fluid and the maximization of the heat transferred by the fluid. The considered objective function is therefore:

$$
J(\Gamma, \boldsymbol{v}(\Gamma), T(\Gamma))=\omega \int_{\Omega_{f}} \sigma_{f}(\boldsymbol{v}, p): \nabla \boldsymbol{v} \mathrm{d} x-(1-\omega) \int_{\Omega_{f}} \rho c_{p} \boldsymbol{v} \cdot \nabla T \mathrm{~d} x,
$$

for some fixed coefficient $\omega \in[0,1]$. Note that the second term in (6.5) rewrites as the heat flux at the outlet upon integration by parts:

$$
\int_{\Omega_{f}} \rho c_{p} \boldsymbol{v} \cdot \nabla T \mathrm{~d} x=\int_{\partial \Omega_{f}} \rho c_{p} T(\boldsymbol{v} \cdot \boldsymbol{n}) \mathrm{d} s=\int_{\partial \Omega_{f}^{N}} \rho c_{p} T(\boldsymbol{v} \cdot \boldsymbol{n}) \mathrm{d} s+\int_{\partial \Omega_{f}^{D}} \rho c_{p} T_{0}\left(\boldsymbol{v}_{0} \cdot \boldsymbol{n}\right) \mathrm{d} s
$$

where the second term is a constant depending on the inlet data. A constraint is imposed on the fraction $\eta_{f}=0.2=\left|\Omega_{f}\right| /|\Omega|$ of the volume of the total domain occupied by the fluid.

For this example, the formulas needed in the calculation of the sensitivity of $J$ are:

$$
\begin{aligned}
\frac{\partial \mathfrak{J}}{\partial \boldsymbol{\theta}}(\boldsymbol{\theta})=\int_{\Omega_{f}}\left[\left(\omega \sigma_{f}(\boldsymbol{v}, p): \nabla \boldsymbol{v}-(1-\omega) \rho c_{p} \boldsymbol{v}\right.\right. & \cdot \nabla T) \operatorname{div}(\boldsymbol{\theta})] \mathrm{d} x \\
& +\int_{\Omega_{f}}\left[-2 \omega \nu e(\boldsymbol{v}): \nabla \boldsymbol{v} \nabla \boldsymbol{\theta}+(1-\omega) \rho c_{p} \boldsymbol{v} \cdot \nabla \boldsymbol{\theta}^{T} \nabla T\right] \mathrm{d} x,
\end{aligned}
$$

$$
\begin{gathered}
\overline{\frac{\partial \mathfrak{J}}{\partial \boldsymbol{\theta}}}(\boldsymbol{\theta})=-\int_{\Gamma}\left(\omega \sigma_{f}(\boldsymbol{v}, p): \nabla \boldsymbol{v}\right)(\boldsymbol{\theta} \cdot \boldsymbol{n}) \mathrm{d} s \\
\frac{\partial \mathfrak{J}}{\partial(\hat{\boldsymbol{v}}, \hat{p})}\left(\boldsymbol{w}^{\prime}, q^{\prime}\right)=\omega \int_{\Omega_{f}} 4 \nu e(\boldsymbol{v}): e\left(\boldsymbol{w}^{\prime}\right) \mathrm{d} x-(1-\omega) \int_{\Omega_{f}} \rho c_{p} \boldsymbol{w}^{\prime} \cdot \nabla T \mathrm{~d} x, \\
\frac{\partial \mathfrak{J}}{\partial \hat{T}}\left(S^{\prime}\right)=-(1-\omega) \int_{\Omega_{f}} \rho c_{p} \boldsymbol{v} \cdot \nabla S^{\prime} \mathrm{d} x .
\end{gathered}
$$

The numerical values of the various parameters involved are reported in Table 2.

We consider two configurations as for the temperature $T_{\text {up }}$ at the upper wall of the device. In a first example (Ex.1), this temperature is lower than the inlet temperature: $T_{\mathrm{up}}<T_{\mathrm{in}}<T_{\text {low }}$. The resulting optimized shape, represented in Figure 12, consists of a main pipe accumulating the hot temperature from the bottom wall with additional outgrowths of fluid domains where the velocity is almost zero. Similar outgrowths have been observed in [37], since they take advantage of the low diffusivity of the fluid material to insulate thermically the main pipe from the colder temperature of the upper wall. Several intermediate shapes arising in the course of the optimization process are depicted on Figure 14.

In a second example (Ex.2), the upper and lower wall temperatures are equal and higher than the inlet temperature $\left(T_{\mathrm{in}}<T_{\mathrm{up}}=T_{\text {low }}\right)$. The optimized shape is represented on Figure 13. Here, the optimization creates oblique areas of fluid with very small velocity so as to insulate thermically the main pipes from the cold inlet temperature. Note that in contrast with [37], the optimization selects a design featuring a lack of symmetry in the test case of Ex.2 (Figure 13), as a consequence of the use of unstructured meshes. 

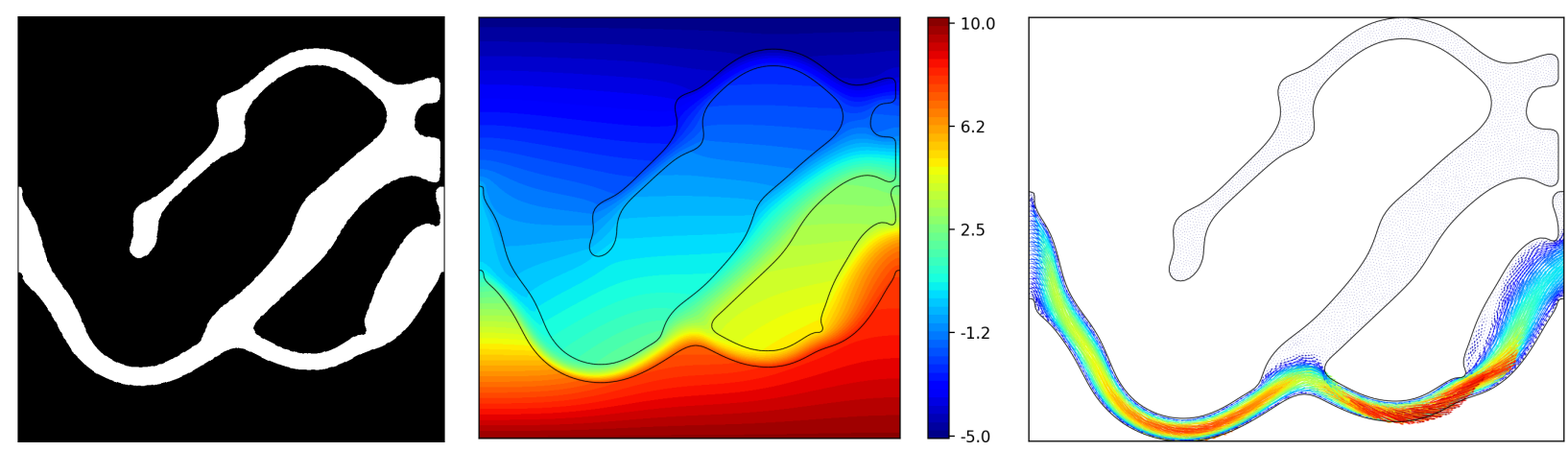

FiguRE 12. Left: optimized configuration for the convective heat transfer test case (Ex.1) of Section 6.3, where the temperature at the upper wall, at the inlet and at the lower wall satisfy $T_{\text {up }}<T_{\text {in }}<T_{\text {low }}$. Middle: corresponding temperature field $T$. Right: fluid velocity field $\boldsymbol{v}$.
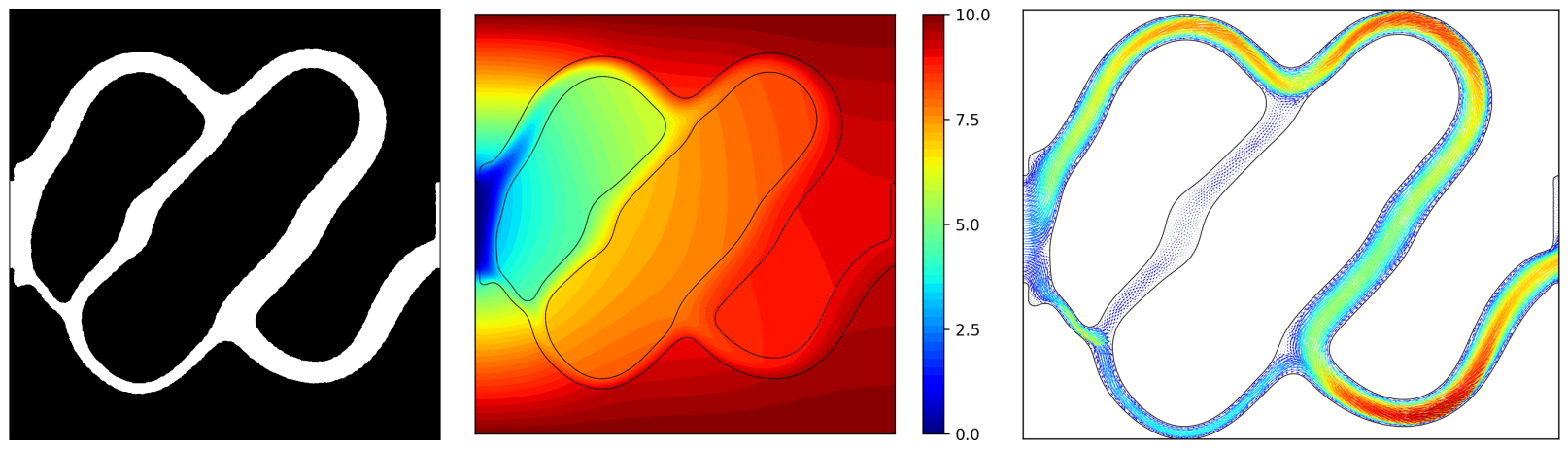

FiguRE 13. Left: optimized configuration for the convective heat transfer test case (Ex.2) of Section 6.3, where the temperature at the upper wall, at the inlet and at the lower wall satisfy $T_{\text {in }}<T_{\text {up }}=T_{\text {low }}$. Middle: corresponding temperature field $T$. Right: fluid velocity field $\boldsymbol{v}$

\subsection{Optimization of a compliant thermoelastic solid with fluid-structure interaction.}

We finally turn to a shape optimization example in the full three-physic setting presented in Section 2. A fluid is flowing from the left to the right of a two-dimensional pipe; at the center of this pipe, a solid body is attached to the boundary of a small non optimizable square $D$ of side length $c$. The flow is entering the pipe at the inlet with a parabolic profile (with maximal velocity $v_{\max }=1$ ), and a prescribed temperature $T_{\mathrm{in}}$ and the solid body receives a thermal flux $h$ applied at the boundary $\partial D$ of the square. The reference temperature of the solid material is equal to the fluid inlet temperature: $T_{\text {ext }}=T_{\mathrm{in}}$. All the other boundaries in this device are insulated from the outside: zero Neumann boundary conditions $\frac{\partial T}{\partial n}=0$ hold as far as the temperature is concerned; see Figure 15 for a schematic of the problem.

We consider in total four different physical configurations corresponding to two possible signs of the thermal flux - either $h>0$ or $h<0$ - and to two different systems for the physical behavior of the flow: either the Navier-Stokes system (2.1), or its linear Stokes counterpart (obtained from the former by omitting the non linear term). In the case where $h>0$, the square boundary $\partial D$ plays the role of a thermal source: the high temperature in the solid body induces thermal expansion. In the latter case, where $h<0, \partial D$ plays the role of a thermal sink: the lower temperature in the solid body induces thermal contraction. In both cases, the role of the fluid is to mitigate the temperature variations induced in $\Omega_{s}$ by the thermal source term $h$. 

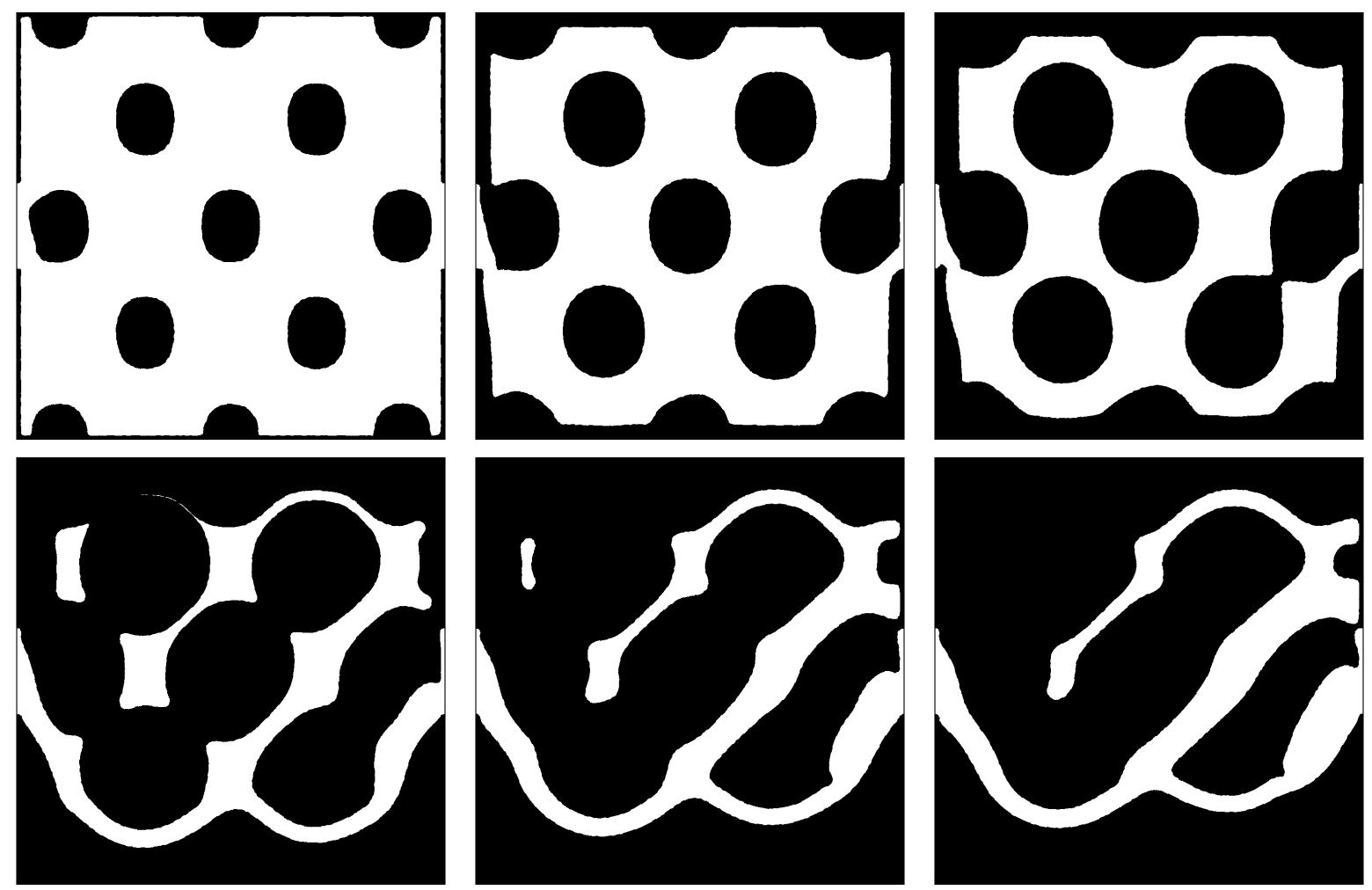

FiguRE 14. From left to right and top to bottom: iterations 1, 15, 30, 110, 220 and 300 of the optimization process in the convective heat transfer of Section 6.3 in the situation (Ex.1).

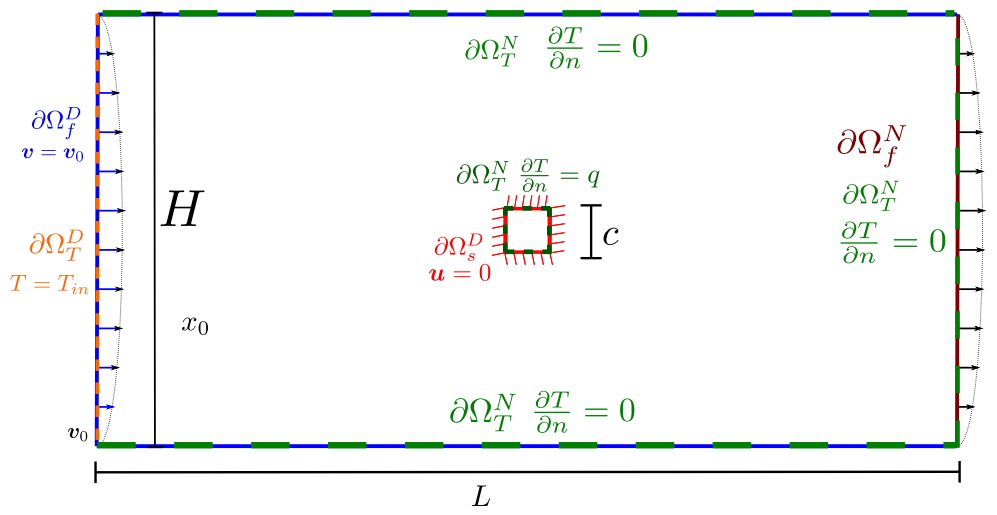

FigURE 15. Setting of the thermoelastic fluid-structure problem of Section 6.4.

The Reynolds number is set to $\mathrm{Re}=\frac{\rho L v_{\max }}{\nu}=200$ and the Péclet number is $\mathrm{Pe}=\frac{L v_{\max }}{k_{f}}=1000$. The volume fraction $\eta_{s}=\left|\Omega_{s}\right| /|\Omega|$ of the solid phase is imposed to be equal to 0.03 . In all the considered regimes, a sufficiently high value of the thermal dilation coefficient $\alpha$ is used so as to make the thermoelastic effect dominant. The various numerical values for the physical parameters of the problem are summarized in Table 3 . 


\begin{tabular}{ccccccccccccccc}
\hline$L$ & $H$ & $c$ & $\rho$ & $c_{p}$ & $\nu$ & $v_{\max }$ & $k_{s}$ & $k_{f}$ & $T_{\mathrm{in}}$ & $T_{\mathrm{ref}}$ & $\lambda$ & $\mu$ & $\alpha$ & $h$ \\
\hline \hline 2 & 1 & 0.1 & 1 & 0.5 & 0.01 & 1 & 10 & 1 & 0 & 0 & 12.96 & 5.55 & 3 & \pm 250 \\
\hline
\end{tabular}

TABLE 3. Parameter values for the thermoelastic fluid-structure test case of Section 6.4.

Our aim is to minimize the mechanical efforts induced in the solid structure $\Omega_{s}$ by the thermal dilation effect and the stress imposed by the fluid:

$$
J(\Gamma, \boldsymbol{u}(\Gamma))=\int_{\Omega_{s}} \sigma_{s}\left(\boldsymbol{u}, T_{s}\right): \nabla \boldsymbol{u} \mathrm{d} x,
$$

which corresponds to the internal energy stored inside the structure. The sensitivity of $J$ with respect to the domain is calculated using the following formulas:

$$
\begin{gathered}
\frac{\partial \mathfrak{J}}{\partial \boldsymbol{\theta}}(\boldsymbol{\theta})=\int_{\Omega_{s}}\left(\sigma_{s}\left(\boldsymbol{u}, T_{s}\right): \nabla \boldsymbol{u} \operatorname{div}(\boldsymbol{\theta})-2 A e(\boldsymbol{u}): \nabla \boldsymbol{u} \nabla \boldsymbol{\theta}+\alpha\left(T_{s}-T_{r e f}\right) \operatorname{Tr}(\nabla \boldsymbol{u} \nabla \boldsymbol{\theta})\right) \mathrm{d} x, \\
\frac{\partial \mathfrak{J}}{\partial \boldsymbol{\theta}}(\boldsymbol{\theta})=-\int_{\Gamma}\left[\sigma_{s}\left(\boldsymbol{u}, T_{s}\right): \nabla \boldsymbol{u}-2 \boldsymbol{n} \cdot A e(\boldsymbol{u}) \nabla \boldsymbol{u} \cdot \boldsymbol{n}+\alpha\left(T_{s}-T_{r e f}\right) \boldsymbol{n} \cdot \nabla \boldsymbol{u} \cdot \boldsymbol{n}\right](\boldsymbol{\theta} \cdot \boldsymbol{n}) \mathrm{d} s \\
\frac{\partial \mathfrak{J}}{\partial \hat{\boldsymbol{u}}}\left(\boldsymbol{r}^{\prime}\right)=\int_{\Omega_{s}}\left(2 A e(\boldsymbol{u}): e\left(\boldsymbol{r}^{\prime}\right)-\alpha\left(T_{s}-T_{r e f}\right) \operatorname{div}\left(\boldsymbol{r}^{\prime}\right)\right) \mathrm{d} x, \\
\frac{\partial \mathfrak{J}}{\partial \hat{T}}\left(S^{\prime}\right)=-\int_{\Omega_{s}} \alpha S^{\prime} \operatorname{div}(\boldsymbol{u}) \mathrm{d} x .
\end{gathered}
$$

The optimized shapes in the four situations are displayed in Figure 16. Note that for the Stokes flow with $h>0$, we used a hole shaped initialization in order to improve the convergence. For the other test cases, we used a disk shape initialization. The convergence histories for the objective function and deviation to the volume constraint are shown on Figure 17. Notably, our optimization algorithm was able to decrease the objective function while keeping the volume fraction constant. Several intermediate shapes are represented in Figure 18 in the situation where $h>0$ and the fluid behavior is driven either by the Stokes or the full Navier-Stokes equations (2.1). For this latter case, the state variables $\boldsymbol{v}, T$ and $\boldsymbol{u}$ are additionnally depicted on Figure 19. In all four cases, the solid part $\Omega_{s}$ tends to have a large contact surface with the fluid, so as to reduce the effect of the thermal source (recall that $T_{\text {in }}=T_{\text {ref }}$ ). The optimized shapes in the cases $h>0$ and $h<0$ are dramatically different, and are quite unintuitive from the mechanical viewpoint. Finally, the optimized shapes for a common value of $h$ are noticeably different between the Stokes and Navier-Stokes cases, which could be expected due to the non negligible value of the Reynolds number. We have indeed checked that the optimized shape in the case of a Stokes flow has worse performance when evaluated in the context of a Navier-Stokes flow than the optimized shape in this setting (and vice-versa).

In this example, the objective function $J$ turns out to be very sensitive with respect to very small variations of the shape. Recall that we do not resort to any upwinding scheme in our implementation. Therefore, we used a very fine mesh resolution (the minimum edge length was $\mathrm{hmin}=0.001$ ) as well as the volume expression (3.36) of the shape derivative, which both enhanced the quality of the optimization process; see Figure 20.

On average, each intermediate mesh of $\Omega$ has approximately 20,000 vertices. A typical 300 iteration run of any of the aforementionned test cases (including Newton iterations for the numerical resolution of the Navier-Stokes equations) took approximately 6 hours on a $2.50 \mathrm{GHz} \operatorname{Intel}(\mathrm{R}) \mathrm{Xeon}(\mathrm{R}) \mathrm{CPU}$.

As we have already mentionned, these are preliminary results. The ongoing work focuses on more realistic test cases, featuring larger Reynolds and Péclet numbers, and possibly in three space dimensions; this will notably require to introduce upwinding in our finite element methods. 


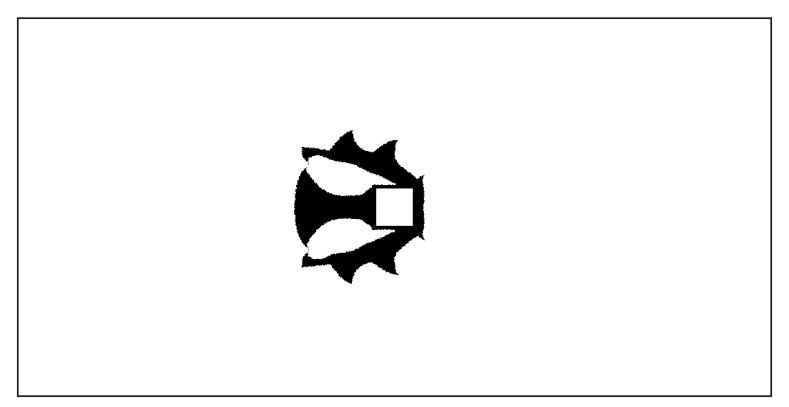

(A) $h>0$ (Stokes)

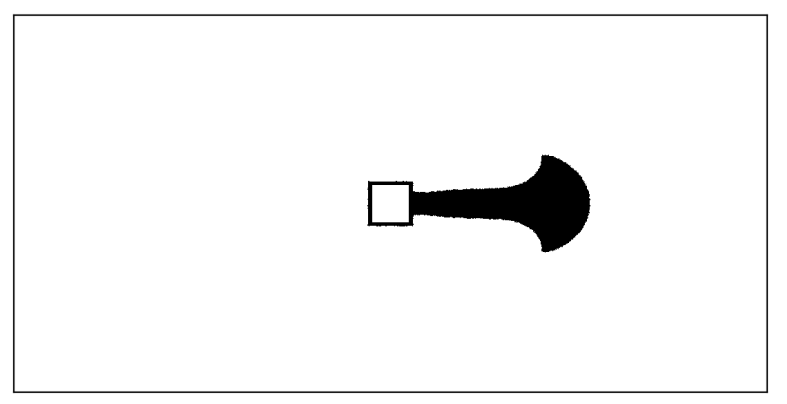

(c) $h<0$ (Stokes)

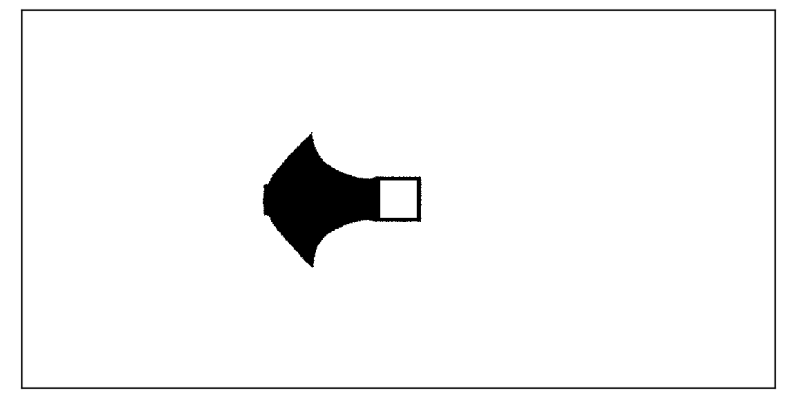

(в) $h>0$ (Navier-Stokes)

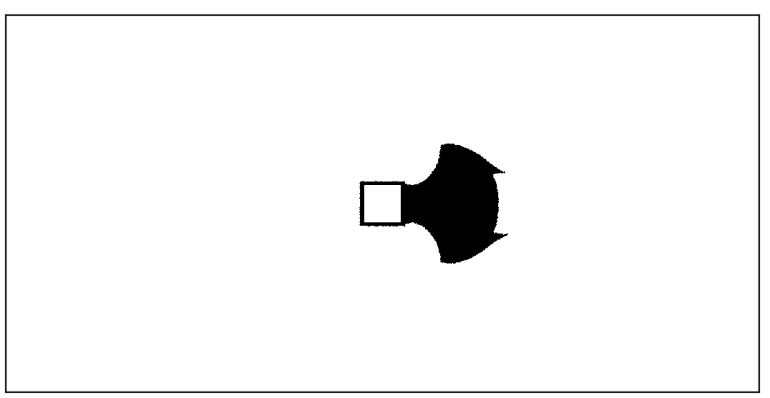

(D) $h<0$ (Navier-Stokes)

Figure 16. Optimized shapes for the three-physic test case of Section 6.4 in the four considered physical situations.

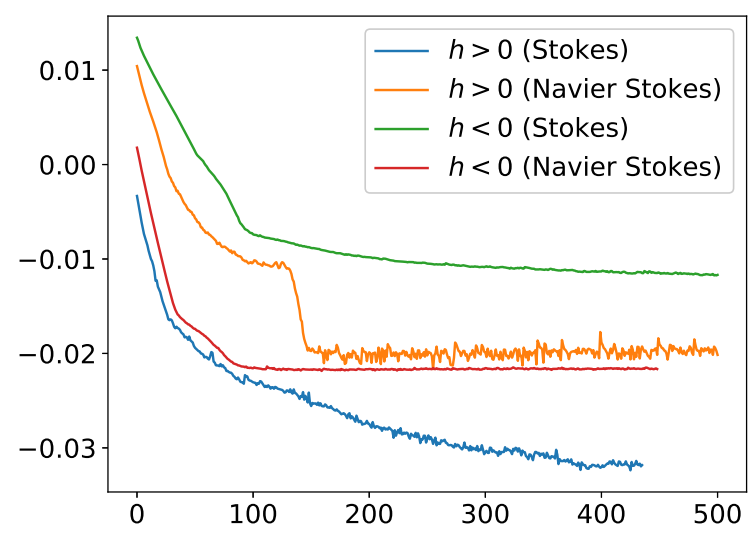

(A) Objective functions $J$

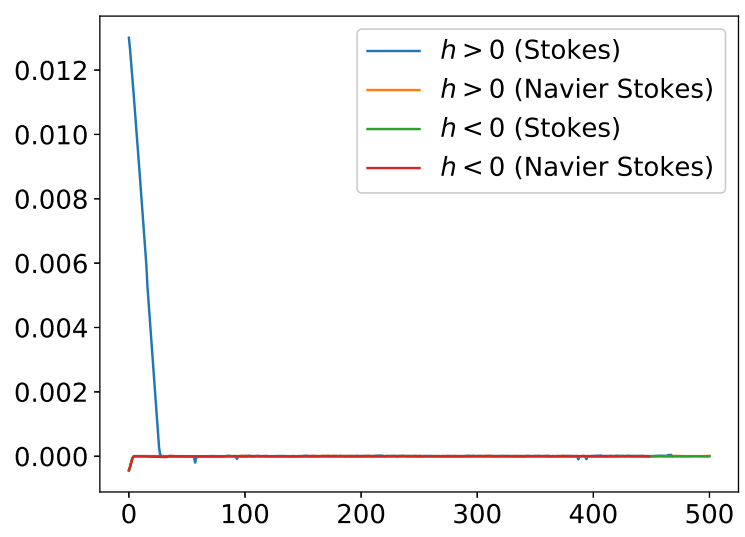

(B) Deviations $\eta_{s}-0.03$ to the volume constraint.

Figure 17. Convergence histories of the objective and constraint functions in the threephysic test case of Section 6.4.

\section{Appendix A. Proof of Proposition 4}

We provide in this appendix a proof of Propositions 4 and 5, or equivalently of (4.14) and (4.15), which is a mere adaptation of the arguments involved in Section 3. Using classical arguments based on the implicit function theorem (see e.g. [32]), one proves that under the condition that the linearized version of the state equations (2.1) to (2.3) are well posed (see Remark 10), the mappings $\boldsymbol{v}\left(\Gamma_{\boldsymbol{\theta}}\right) \circ(I+\boldsymbol{\theta}), p\left(\Gamma_{\boldsymbol{\theta}}\right) \circ(I+\boldsymbol{\theta})$, $T\left(\Gamma_{\boldsymbol{\theta}}\right) \circ(I+\boldsymbol{\theta})$, and $\boldsymbol{u}\left(\Gamma_{\boldsymbol{\theta}}\right) \circ(I+\boldsymbol{\theta})$ are differentiable with respect to $\boldsymbol{\theta}$. Differentiating the variational formulations (4.2) to (4.4), one finds that the Fréchet derivatives $\dot{\boldsymbol{v}}(\boldsymbol{\theta}), \dot{p}(\boldsymbol{\theta}), \dot{T}(\boldsymbol{\theta})$ and $\dot{\boldsymbol{u}}(\boldsymbol{\theta})$ at $\boldsymbol{\theta}=0$ solve 

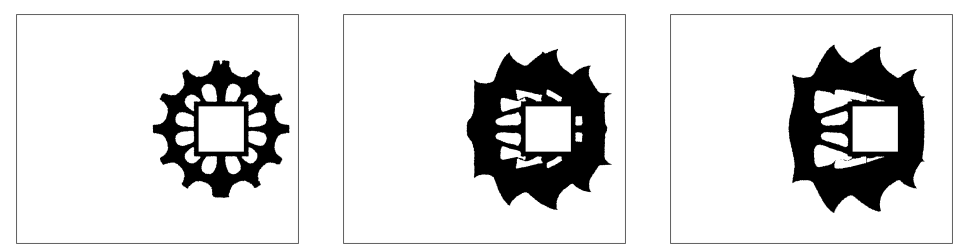

(A) $h>0$ (Stokes)
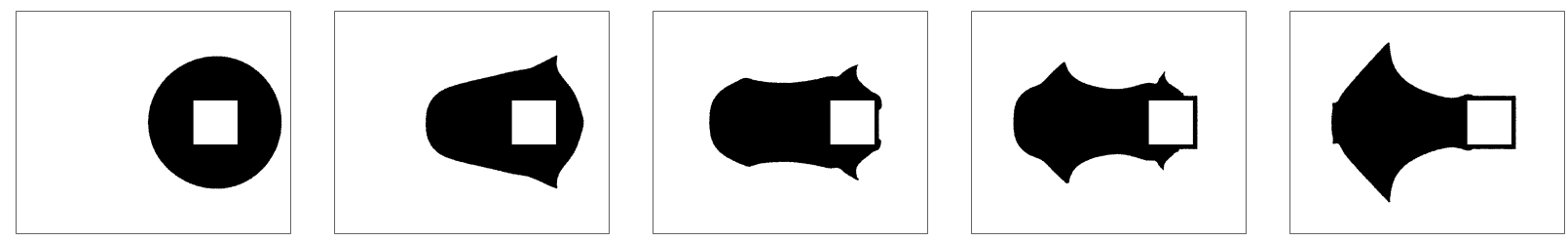

(B) $h>0$ (Navier-Stokes)

Figure 18. From left to right and top to bottom: iterations 1, 35, 80, 120 and 300 of the optimization process in the three-physic context of Section 6.4 for Stokes and Navier Stokes flow where $h>0$.

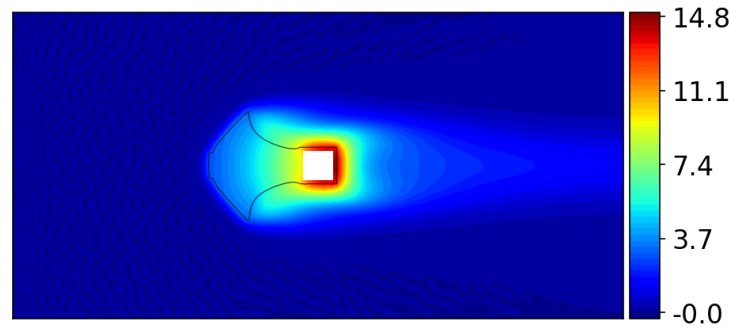

(A) Temperature field $T$

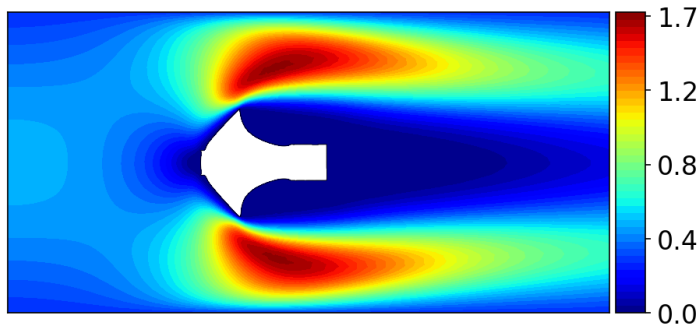

(B) Velocity field $\boldsymbol{v}$ (kinetic energy $\left(v_{x}^{2}+v_{y}^{2}\right) / 2$ )

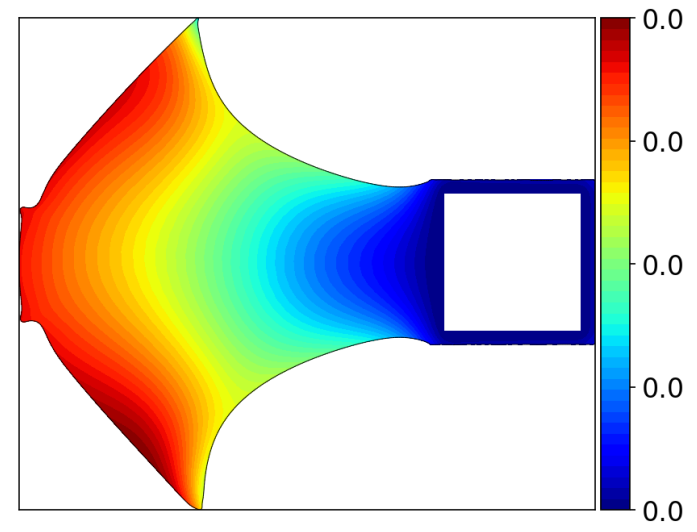

(C) Displacement field $\boldsymbol{u}$ (square energy $\left(u_{x}^{2}+u_{y}^{2}\right) / 2$ )

Figure 19. State variables $\boldsymbol{v}, T$ and $\boldsymbol{u}$ for the optimized configuration of the three-physic shape optimization problem of Section 6.4, in the situation $h>0$ and solved with the Navier-Stokes equations.

the following variational problems:

(A.1) Find $(\dot{\boldsymbol{v}}(\boldsymbol{\theta}), \dot{p}(\boldsymbol{\theta})) \in V_{\boldsymbol{v}, p}(\Gamma)$ such that $\forall\left(\boldsymbol{w}^{\prime}, q^{\prime}\right) \in V_{\boldsymbol{v}, p}(\Gamma)$,

$$
\begin{aligned}
& \int_{\Omega_{f}}\left[\sigma_{f}(\dot{\boldsymbol{v}}(\boldsymbol{\theta}), \dot{p}(\boldsymbol{\theta}))\right.\left.: \nabla \boldsymbol{w}^{\prime}+\rho \boldsymbol{w}^{\prime} \cdot \nabla \boldsymbol{v} \cdot \dot{\boldsymbol{v}}(\boldsymbol{\theta})+\rho \boldsymbol{w}^{\prime} \cdot \nabla \dot{\boldsymbol{v}}(\boldsymbol{\theta}) \cdot \boldsymbol{v}-q^{\prime} \operatorname{div}(\dot{\boldsymbol{v}}(\boldsymbol{\theta}))\right] \mathrm{d} x \\
&=\int_{\Omega_{f}}\left[\boldsymbol{w}^{\prime} \cdot \operatorname{div}\left(\boldsymbol{f}_{f} \otimes \boldsymbol{\theta}\right)-\left(\sigma_{f}(\boldsymbol{v}, p): \nabla \boldsymbol{w}^{\prime}+\rho \boldsymbol{w}^{\prime} \cdot \nabla \boldsymbol{v} \cdot \boldsymbol{v}\right) \operatorname{div}(\boldsymbol{\theta})\right] \mathrm{d} x \\
& \quad+\int_{\Omega_{f}}\left(\sigma_{f}(\boldsymbol{v}, p):\left(\nabla \boldsymbol{w}^{\prime} \nabla \boldsymbol{\theta}\right)+\sigma_{f}\left(\boldsymbol{w}^{\prime}, q^{\prime}\right):(\nabla \boldsymbol{v} \nabla \boldsymbol{\theta})+\rho \boldsymbol{w}^{\prime} \cdot \nabla \boldsymbol{v} \nabla \boldsymbol{\theta} \cdot \boldsymbol{v}\right) \mathrm{d} x,
\end{aligned}
$$




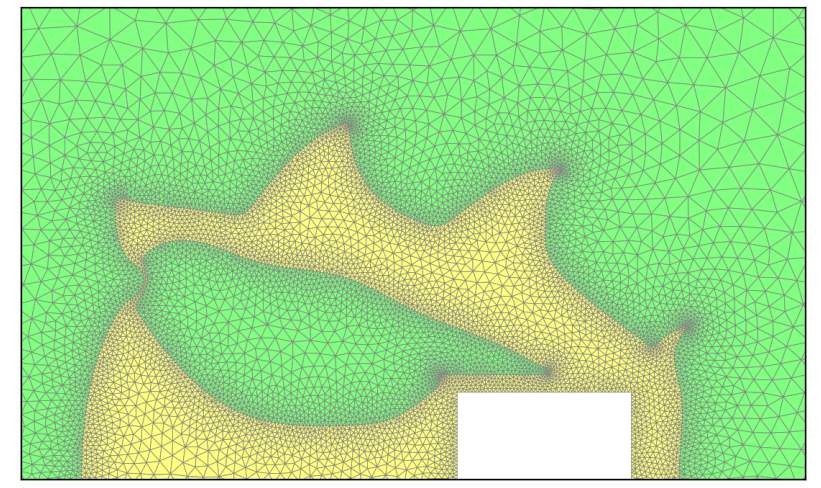

Figure 20. Zooms on the mesh for the final configuration of the Stokes case of Ex. 1.

(A.2) Find $\dot{T}(\boldsymbol{\theta}) \in V_{T}(\Gamma)$ such that $\forall S^{\prime} \in V_{T}(\Gamma)$,

$$
\begin{aligned}
\int_{\Omega_{s}} k_{s} \nabla \dot{T}(\boldsymbol{\theta}) \cdot \nabla & S^{\prime} \mathrm{d} x+\int_{\Omega_{f}}\left(k_{f} \nabla \dot{T}(\boldsymbol{\theta}) \cdot \nabla S+\rho c_{p} S^{\prime} \dot{\boldsymbol{v}}(\boldsymbol{\theta}) \cdot \nabla T\right) \mathrm{d} x=-\int_{\Omega_{f}} \rho c_{p} S^{\prime} \boldsymbol{v} \cdot \nabla \dot{T}(\boldsymbol{\theta}) \mathrm{d} x \\
+ & \int_{\Omega_{s}}\left[\operatorname{div}\left(Q_{s} \boldsymbol{\theta}\right) S^{\prime}+k_{s}\left(\nabla \boldsymbol{\theta}+\nabla \boldsymbol{\theta}^{T}-\operatorname{div}(\boldsymbol{\theta}) I\right) \nabla T \cdot \nabla S^{\prime}\right] \mathrm{d} x \\
+ & \int_{\Omega_{f}}\left[\operatorname{div}\left(Q_{f} \boldsymbol{\theta}\right) S^{\prime}+k_{f}\left(\nabla \boldsymbol{\theta}+\nabla \boldsymbol{\theta}^{T}-\operatorname{div}(\boldsymbol{\theta}) I\right) \nabla T \cdot \nabla S^{\prime}\right] \mathrm{d} x \\
& +\int_{\Omega_{f}}\left(-\rho c_{p} S^{\prime} \boldsymbol{v} \cdot \nabla T \operatorname{div}(\boldsymbol{\theta})+\rho c_{p} S^{\prime} \boldsymbol{v} \cdot \nabla \boldsymbol{\theta}^{T} \nabla T\right) \mathrm{d} x
\end{aligned}
$$

(A.3) Find $\dot{\boldsymbol{u}}(\boldsymbol{\theta}) \in V_{\boldsymbol{u}}(\Gamma)$ such that $\forall \boldsymbol{r}^{\prime} \in V_{\boldsymbol{u}}(\Gamma)$,

$$
\begin{array}{rl}
\int_{\Omega_{s}} A e(\dot{\boldsymbol{u}}(\boldsymbol{\theta})): \nabla \boldsymbol{r}^{\prime} \mathrm{d} & x=\int_{\Omega_{s}} \alpha \dot{T}(\boldsymbol{\theta}) \operatorname{div}\left(\boldsymbol{r}^{\prime}\right) \mathrm{d} x+\int_{\Omega_{s}}\left[-\operatorname{div}(\boldsymbol{\theta}) \sigma_{s}\left(\boldsymbol{u}, T_{s}\right): \nabla \boldsymbol{r}^{\prime}+\operatorname{div}\left(\boldsymbol{f}_{s} \otimes \boldsymbol{\theta}\right) \cdot \boldsymbol{r}^{\prime}\right] \mathrm{d} x \\
& +\int_{\Omega_{s}}\left(\sigma_{s}\left(\boldsymbol{u}, T_{s}\right):(\nabla \boldsymbol{r} \nabla \boldsymbol{\theta})+A e(\boldsymbol{r}):(\nabla \boldsymbol{u} \nabla \boldsymbol{\theta})\right) \mathrm{d} x-\int_{\Gamma} \boldsymbol{r}^{\prime} \cdot \sigma_{f}(\dot{\boldsymbol{v}}(\boldsymbol{\theta}), \dot{p}(\boldsymbol{\theta})) \cdot \boldsymbol{n} \mathrm{d} s,
\end{array}
$$

where $\sigma_{f}(\dot{\boldsymbol{v}}(\boldsymbol{\theta}), \dot{p}(\boldsymbol{\theta})) \cdot \boldsymbol{n}$ is an element of the dual space $H_{00}^{-1 / 2}\left(\Gamma, \mathbb{R}^{d}\right)$ of $H_{00}^{1 / 2}\left(\Gamma, \mathbb{R}^{d}\right)$ whose action is given by (differentiating (4.5) with respect to $\boldsymbol{\theta})$ :

$$
\begin{aligned}
& \forall \boldsymbol{r}^{\prime} \in H_{00}^{1 / 2}\left(\Gamma, \mathbb{R}^{d}\right),-\int_{\Gamma} \boldsymbol{r}^{\prime} \cdot \sigma_{f}(\dot{\boldsymbol{v}}(\boldsymbol{\theta}), \dot{p}(\boldsymbol{\theta})) \cdot \boldsymbol{n} \mathrm{d} s \\
&=\int_{\Omega_{f}}\left(\operatorname{div}\left(\boldsymbol{f}_{f} \otimes \boldsymbol{\theta}\right) \cdot \tilde{\boldsymbol{r}}-\left(\rho \tilde{\boldsymbol{r}} \cdot \nabla \boldsymbol{v} \cdot \boldsymbol{v}+\sigma_{f}(\boldsymbol{v}, p): \nabla \tilde{\boldsymbol{r}}\right) \operatorname{div}(\boldsymbol{\theta})\right) \mathrm{d} x \\
&+\int_{\Omega_{f}}\left(\rho \tilde{\boldsymbol{r}} \cdot \nabla \boldsymbol{v} \nabla \boldsymbol{\theta} \cdot \boldsymbol{v}+\sigma_{f}(\boldsymbol{v}, p):(\nabla \tilde{\boldsymbol{r}} \nabla \boldsymbol{\theta})+\sigma_{f}(\tilde{\boldsymbol{r}}, \tilde{q}):(\nabla \boldsymbol{v} \nabla \boldsymbol{\theta})\right) \mathrm{d} x \\
& \quad-\int_{\Omega_{f}}\left(\sigma_{f}(\dot{\boldsymbol{v}}(\boldsymbol{\theta}), \dot{p}(\boldsymbol{\theta})): \nabla \tilde{\boldsymbol{r}}+\rho \tilde{\boldsymbol{r}} \cdot \nabla \boldsymbol{v} \cdot \dot{\boldsymbol{v}}(\boldsymbol{\theta})+\rho \tilde{\boldsymbol{r}} \cdot \nabla \dot{\boldsymbol{v}}(\boldsymbol{\theta}) \cdot \boldsymbol{v}-\tilde{q} \operatorname{div}(\dot{\boldsymbol{v}}(\boldsymbol{\theta}))\right) \mathrm{d} x,
\end{aligned}
$$

for any extension $(\tilde{\boldsymbol{r}}, \tilde{q}) \in V_{\boldsymbol{v}, p}(\Gamma)$ satisfying $\tilde{\boldsymbol{r}}=\boldsymbol{r}^{\prime}$ on $\Gamma$. Note that the above expression is independent of the extension because of (A.1) with $\boldsymbol{w}^{\prime}=\tilde{\boldsymbol{r}}$ and $q^{\prime}=\tilde{q}$. Then by definition of $\mathfrak{J}$ :

$$
\begin{aligned}
J\left(\Gamma_{\boldsymbol{\theta}}, \boldsymbol{v}\left(\Gamma_{\boldsymbol{\theta}}\right), p\left(\Gamma_{\boldsymbol{\theta}}\right), T\left(\Gamma_{\boldsymbol{\theta}}\right),\right. & \left.\boldsymbol{u}\left(\Gamma_{\boldsymbol{\theta}}\right)\right) \\
& =\mathfrak{J}\left(\boldsymbol{\theta}, \boldsymbol{v}\left(\Gamma_{\boldsymbol{\theta}}\right) \circ(I+\boldsymbol{\theta}), p\left(\Gamma_{\boldsymbol{\theta}}\right) \circ(I+\boldsymbol{\theta}), T\left(\Gamma_{\boldsymbol{\theta}}\right) \circ(I+\boldsymbol{\theta}), \boldsymbol{u}\left(\Gamma_{\boldsymbol{\theta}}\right) \circ(I+\boldsymbol{\theta})\right),
\end{aligned}
$$


whence the chain rule yields:

$$
\frac{\mathrm{d}}{\mathrm{d} \boldsymbol{\theta}}\left[J\left(\Gamma_{\boldsymbol{\theta}}, \boldsymbol{v}\left(\Gamma_{\boldsymbol{\theta}}\right), p\left(\Gamma_{\boldsymbol{\theta}}\right), T\left(\Gamma_{\boldsymbol{\theta}}\right), \boldsymbol{u}\left(\Gamma_{\boldsymbol{\theta}}\right)\right)\right](\boldsymbol{\theta})=\frac{\partial \mathfrak{J}}{\partial \boldsymbol{\theta}}(\boldsymbol{\theta})+\frac{\partial \mathfrak{J}}{\partial(\boldsymbol{v}, p)}(\dot{\boldsymbol{v}}(\boldsymbol{\theta}), \dot{p}(\boldsymbol{\theta}))+\frac{\partial \mathfrak{J}}{\partial T}(\dot{T}(\boldsymbol{\theta}))+\frac{\partial \mathfrak{J}}{\partial \boldsymbol{u}}(\boldsymbol{u}(\boldsymbol{\theta}))
$$

One then uses the adjoint equations (4.9) to (4.11) with $\boldsymbol{r}^{\prime}=\dot{\boldsymbol{u}}(\boldsymbol{\theta}), S^{\prime}=\dot{T}(\boldsymbol{\theta}), \boldsymbol{w}^{\prime}=\dot{\boldsymbol{v}}(\boldsymbol{\theta}), q^{\prime}=\dot{p}(\boldsymbol{\theta})$ as test functions to obtain:

$$
\frac{\partial \mathfrak{J}}{\partial T}(\dot{T}(\boldsymbol{\theta}))=\int_{\Omega_{s}} k_{s} \nabla S \cdot \nabla \dot{T}(\boldsymbol{\theta}) \mathrm{d} x+\int_{\Omega_{f}}\left(k_{f} \nabla S \cdot \nabla \dot{T}(\boldsymbol{\theta})+\rho c_{p} S \boldsymbol{v} \cdot \nabla \dot{T}(\boldsymbol{\theta})\right) \mathrm{d} x-\int_{\Omega_{s}} \alpha \dot{T}(\boldsymbol{\theta}) \operatorname{div}(\boldsymbol{r}) \mathrm{d} x,
$$

$$
\begin{aligned}
& \frac{\partial \mathfrak{J}}{\partial(\boldsymbol{v}, p)}(\dot{\boldsymbol{v}}(\boldsymbol{\theta}), \dot{p}(\boldsymbol{\theta})) \\
= & \int_{\Omega_{f}}\left(\sigma_{f}(\boldsymbol{w}, q): \nabla \dot{\boldsymbol{v}}(\boldsymbol{\theta})+\rho \boldsymbol{w} \cdot \nabla \boldsymbol{v} \cdot \dot{\boldsymbol{v}}(\boldsymbol{\theta})+\rho \boldsymbol{w} \cdot \nabla \dot{\boldsymbol{v}}(\boldsymbol{\theta}) \cdot \boldsymbol{v}-\dot{p}(\boldsymbol{\theta}) \operatorname{div}(\boldsymbol{w})\right) \mathrm{d} x+\int_{\Omega_{f}} \rho c_{p} S \nabla T \cdot \dot{\boldsymbol{v}}(\boldsymbol{\theta}) \mathrm{d} x \\
= & \int_{\Omega_{f}}\left(\sigma_{f}(\dot{\boldsymbol{v}}(\boldsymbol{\theta}), \dot{p}(\boldsymbol{\theta})): \nabla \boldsymbol{w}+\rho \boldsymbol{w} \cdot \nabla \dot{\boldsymbol{v}}(\boldsymbol{\theta}) \cdot \boldsymbol{v}+\rho \boldsymbol{w} \cdot \nabla \boldsymbol{v} \cdot \dot{\boldsymbol{v}}(\boldsymbol{\theta})-q \operatorname{div}(\dot{\boldsymbol{v}}(\boldsymbol{\theta}))\right) \mathrm{d} x+\int_{\Omega_{f}} \rho c_{p} S \nabla T \cdot \dot{\boldsymbol{v}}(\boldsymbol{\theta}) \mathrm{d} x .
\end{aligned}
$$

Using now equations (A.2) and (A.3) with $\boldsymbol{r}^{\prime}=\boldsymbol{r}, S^{\prime}=S$ as test functions and (A.4) with $(\tilde{\boldsymbol{r}}, \tilde{q})=(\boldsymbol{w}, q)$ as an extension of $\boldsymbol{r}^{\prime}=\boldsymbol{r} \in H^{1 / 2}\left(\Gamma, \mathbb{R}^{d}\right)$ to eliminate the bilinear terms, the above three equations rewrite:

$$
\begin{aligned}
\frac{\partial \mathfrak{J}}{\partial \boldsymbol{u}}(\dot{\boldsymbol{u}}(\boldsymbol{\theta}))=-\int_{\Gamma} \boldsymbol{r} & \sigma_{f}(\dot{\boldsymbol{v}}(\boldsymbol{\theta}), \dot{p}(\boldsymbol{\theta})) \cdot \boldsymbol{n} \mathrm{d} s+\int_{\Omega_{s}} \alpha \dot{T}(\boldsymbol{\theta}) \operatorname{div}(\boldsymbol{r}) \mathrm{d} x \\
+\int_{\Omega_{s}}\left[-\operatorname{div}(\boldsymbol{\theta}) \sigma_{s}\left(\boldsymbol{u}, T_{s}\right):\right. & \left.\nabla \boldsymbol{r}+\operatorname{div}\left(\boldsymbol{f}_{s} \otimes \boldsymbol{\theta}\right) \cdot \boldsymbol{r}\right] \mathrm{d} x \\
& +\int_{\Omega_{s}}\left(\sigma_{s}\left(\boldsymbol{u}, T_{s}\right):(\nabla \boldsymbol{r} \nabla \boldsymbol{\theta})+A e(\boldsymbol{r}):(\nabla \boldsymbol{u} \nabla \boldsymbol{\theta})\right) \mathrm{d} x,
\end{aligned}
$$

$$
\begin{aligned}
& \frac{\partial \mathfrak{J}}{\partial T}(\dot{T}(\boldsymbol{\theta}))=- \int_{\Omega_{s}} \alpha \dot{T}(\boldsymbol{\theta}) \operatorname{div}(\boldsymbol{r}) \mathrm{d} x-\int_{\Omega_{f}} \rho c_{p} S \dot{\boldsymbol{v}}(\boldsymbol{\theta}) \cdot \nabla T \mathrm{~d} x \\
&+\int_{\Omega_{s}}\left[\operatorname{div}\left(Q_{s} \boldsymbol{\theta}\right) S+k_{s}\left(\nabla \boldsymbol{\theta}+\nabla \boldsymbol{\theta}^{T}-\operatorname{div}(\boldsymbol{\theta}) I\right) \nabla T \cdot \nabla S\right] \mathrm{d} x \\
&+\int_{\Omega_{f}}\left[\operatorname{div}\left(Q_{f} \boldsymbol{\theta}\right) S+k_{f}\left(\nabla \boldsymbol{\theta}+\nabla \boldsymbol{\theta}^{T}-\operatorname{div}(\boldsymbol{\theta}) I\right) \nabla T \cdot \nabla S\right] \mathrm{d} x \\
&+\int_{\Omega_{f}}\left(-\rho c_{p} S \boldsymbol{v} \cdot \nabla T \operatorname{div}(\boldsymbol{\theta})+\rho c_{p} S \boldsymbol{v} \cdot \nabla \boldsymbol{\theta}^{T} \nabla T\right) \mathrm{d} x \\
& \frac{\partial \mathfrak{J}}{\partial(\boldsymbol{v}, p)}(\dot{\boldsymbol{v}}(\boldsymbol{\theta}), \dot{p}(\boldsymbol{\theta}))=\int_{\Gamma} \boldsymbol{r} \cdot \sigma_{f}(\dot{\boldsymbol{v}}(\boldsymbol{\theta}), \dot{p}(\boldsymbol{\theta})) \cdot \boldsymbol{n} \mathrm{d} s+\int_{\Omega_{f}} \rho c_{p} S \nabla T \cdot \dot{\boldsymbol{v}}(\boldsymbol{\theta}) \mathrm{d} x \\
&+\int_{\Omega_{f}}\left(\operatorname{div}\left(\boldsymbol{f}_{f} \otimes \boldsymbol{\theta}\right) \cdot \boldsymbol{w}-\left(\rho \boldsymbol{w} \cdot \nabla \boldsymbol{v} \cdot \boldsymbol{v}+\sigma_{f}(\boldsymbol{v}, p): \nabla \boldsymbol{w}\right) \operatorname{div}(\boldsymbol{\theta})\right) \mathrm{d} x \\
& \quad+\int_{\Omega_{f}}\left(\rho \boldsymbol{w} \cdot \nabla \boldsymbol{v} \nabla \boldsymbol{\theta} \cdot \boldsymbol{v}+\sigma_{f}(\boldsymbol{v}, p):(\nabla \boldsymbol{w} \nabla \boldsymbol{\theta})+\sigma_{f}(\boldsymbol{w}, q):(\nabla \boldsymbol{v} \nabla \boldsymbol{\theta})\right) \mathrm{d} x .
\end{aligned}
$$

Formula (4.14) follows by summing up the above three equations. If $H^{2}$ regularity holds for $\boldsymbol{v}, \boldsymbol{u}, T$ and $H^{1}$ regularity holds for $p$ on their respective domains of definition, then an integration by parts allows to rewrite 
(4.14) as;

$$
\begin{aligned}
& \frac{\mathrm{d}}{\mathrm{d} \boldsymbol{\theta}}\left[J\left(\Gamma_{\boldsymbol{\theta}}, \boldsymbol{v}\left(\Gamma_{\boldsymbol{\theta}}\right), p\left(\Gamma_{\boldsymbol{\theta}}\right), T\left(\Gamma_{\boldsymbol{\theta}}\right), \boldsymbol{u}\left(\Gamma_{\boldsymbol{\theta}}\right)\right)\right](\boldsymbol{\theta}) \\
& =\int_{\Gamma} \boldsymbol{g}_{\mathfrak{J}} \cdot \boldsymbol{\theta} \mathrm{d} s+\int_{\Gamma}\left(\boldsymbol{f}_{f} \cdot \boldsymbol{w}-\sigma_{f}(\boldsymbol{v}, p): \nabla \boldsymbol{w}-\rho \boldsymbol{w} \cdot \nabla \boldsymbol{v} \cdot \boldsymbol{v}\right)(\boldsymbol{\theta} \cdot \boldsymbol{n}) \mathrm{d} s \\
& +\int_{\Gamma}\left[\boldsymbol{n} \cdot \sigma_{f}(\boldsymbol{v}, p) \nabla \boldsymbol{w} \cdot \boldsymbol{\theta}+\boldsymbol{n} \cdot \sigma_{f}(\boldsymbol{w}, q) \nabla \boldsymbol{v} \cdot \boldsymbol{\theta}+\rho(\boldsymbol{v} \cdot \boldsymbol{n}) \boldsymbol{w} \cdot \nabla \boldsymbol{v} \cdot \boldsymbol{\theta}\right] \mathrm{d} s \\
& \quad+\int_{\Gamma}\left(k_{s} \nabla T_{s} \cdot \nabla S_{s}-k_{f} \nabla T_{f} \cdot \nabla S_{f}+Q_{f} S-Q_{s} S_{s}\right)(\boldsymbol{\theta} \cdot \boldsymbol{n}) \mathrm{d} s \\
& \quad+\int_{\Gamma}\left(-k_{s}\left(\nabla T_{s} \cdot \boldsymbol{\theta}\right)\left(\nabla S_{s} \cdot \boldsymbol{n}\right)-k_{s}\left(\nabla S_{s} \cdot \boldsymbol{\theta}\right)\left(\nabla T_{s} \cdot \boldsymbol{n}\right)\right) \mathrm{d} s \\
& \quad+\int_{\Gamma}\left(k_{f}\left(\nabla T_{f} \cdot \boldsymbol{\theta}\right)\left(\nabla S_{f} \cdot \boldsymbol{n}\right)+k_{f}\left(\nabla S_{f} \cdot \boldsymbol{\theta}\right)\left(\nabla T_{f} \cdot \boldsymbol{n}\right)\right) \mathrm{d} s \\
& \quad+\int_{\Gamma}\left[\left(\sigma_{s}\left(\boldsymbol{u}, T_{s}\right): \nabla \boldsymbol{r}-\boldsymbol{f}_{s} \cdot \boldsymbol{r}\right)(\boldsymbol{\theta} \cdot \boldsymbol{n})-\boldsymbol{n} \cdot \sigma_{s}\left(\boldsymbol{u}, T_{s}\right) \nabla \boldsymbol{r} \cdot \boldsymbol{\theta}-\boldsymbol{n} \cdot A e(\boldsymbol{r}) \nabla \boldsymbol{u} \cdot \boldsymbol{\theta}\right] \mathrm{d} s+\int_{\Gamma} \boldsymbol{\Lambda} \cdot \boldsymbol{\theta} \mathrm{d} x
\end{aligned}
$$

where $\boldsymbol{\Lambda}$ is a $L^{1}\left(\Omega, \mathbb{R}^{d}\right)$ function obtained from Green's identity. The Hadamard structure theorem implies that (A.13) vanishes on compactly supported fields $\boldsymbol{\theta}$ or on fields $\boldsymbol{\theta}$ tangent to $\Gamma$. This implies that in fact, $\boldsymbol{\Lambda}=0$, and (4.15) follows by removing terms depending on the tangential component of $\boldsymbol{\theta}$ on $\Gamma$.

Acknowledgements. This work was supported by the Association Nationale de la Recherche et de la Technologie (ANRT) [grant number CIFRE 2017/0024]. G. A. is a member of the DEFI project at INRIA Saclay Ile-de-France. The work of G. A. is partially supported by the SOFIA project, funded by BPI (Banque Publique d'Investissement). The work of C.D. is partially supported by the IRS-CAOS grant from Université Grenoble-Alpes.

\section{REFERENCES}

[1] G. Allaire, Conception optimale de structures, volume 58 of Mathématiques \& Applications (Berlin)[Mathematics 83 Applications], Springer-Verlag, Berlin, 2007.

[2] — Shape optimization by the homogenization method, vol. 146, Springer Science \& Business Media, 2012.

[3] G. Allaire, C. Dapogny, And P. Frey, A mesh evolution algorithm based on the level set method for geometry and topology optimization, Structural and Multidisciplinary Optimization, 48 (2013), pp. 711-715.

[4] - Shape optimization with a level set based mesh evolution method, Computer Methods in Applied Mechanics and Engineering, 282 (2014), pp. 22-53.

[5] G. Allaire, F. Jouve, And A. Toader, A level-set method for shape optimization, Comptes Rendus Mathematique, 334 (2002), pp. 1125-1130.

[6] G. Allaire, F. Jouve, And A.-M. Toader, Structural optimization using sensitivity analysis and a level-set method, Journal of Computational Physics, 194 (2004), pp. 363-393.

[7] G. Allaire and O. Pantz, Structural optimization with freefem ++ , Structural and Multidisciplinary Optimization, 32 (2006), pp. 173-181.

[8] M. P. Bendsoe and O. Sigmund, Topology optimization, Springer-Verlag, Berlin, 2003. Theory, methods and applications.

[9] T. Borrvall and J. Petersson, Topology optimization of fluids in stokes flow, International Journal for Numerical Methods in Fluids, 41 (2003), pp. 77-107.

[10] B. Bourdin And A. Chambolle, Design-dependent loads in topology optimization, ESAIM: Control, Optimisation and Calculus of Variations, 9 (2003), pp. 19-48.

[11] F. BOYER ET AL., Trace theorems and spatial continuity properties for the solutions of the transport equation, Differential and integral equations, 18 (2005), pp. 891-934.

[12] H. Brezis, Functional analysis, Sobolev spaces and partial differential equations, Springer Science \& Business Media, 2010.

[13] C. Bui, C. DApogny, And P. Frey, An accurate anisotropic adaptation method for solving the level set advection equation, International Journal for Numerical Methods in Fluids, 70 (2012), pp. 899-922.

[14] M. Burger, A framework for the construction of level set methods for shape optimization and reconstruction, Interfaces and Free boundaries, 5 (2003), pp. 301-329.

[15] J. CÉA, Conception optimale ou identification de formes, calcul rapide de la dérivée directionnelle de la fonction coût, ESAIM: Mathematical Modelling and Numerical Analysis, 20 (1986), pp. 371-402. 
[16] C. P. Chukwudozie, Shape optimization for drag minimization using the Navier-Stokes equation, Master's thesis, Louisiana State University, 2015.

[17] C. Dapogny, Optimisation de formes, méthode des lignes de niveaux sur maillages non structurés et évolution de maillages, PhD thesis, Université Pierre et Marie Curie-Paris VI, 2013.

[18] C. Dapogny, C. Dobrzynski, And P. Frey, Three-dimensional adaptive domain remeshing, implicit domain meshing, and applications to free and moving boundary problems, Journal of Computational Physics, 262 (2014), pp. 358-378.

[19] C. Dapogny, A. Faure, G. Michailidis, G. Allaire, A. Couvelas, and R. Estevez, Geometric constraints for shape and topology optimization in architectural design, Computational Mechanics, 59 (2017), pp. 933-965.

[20] C. Dapogny And P. Frey, Computation of the signed distance function to a discrete contour on adapted triangulation, Calcolo, 49 (2012), pp. 193-219.

[21] C. Dapogny, P. Frey, F. Omnès, and Y. Privat, Geometrical shape optimization in fluid mechanics using FreeFem++. HAL preprint hal-01481707, Mar. 2017.

[22] Т. Dвоuк, A review about the engineering design of optimal heat transfer systems using topology optimization, Applied Thermal Engineering, 112 (2017), pp. 841-854.

[23] F. DE GOURnAY, Velocity extension for the level-set method and multiple eigenvalues in shape optimization, SIAM journal on control and optimization, 45 (2006), pp. 343-367.

[24] M. C. Delfour AND J.-P. ZolÉSIO, Shapes and geometries: metrics, analysis, differential calculus, and optimization, SIAM, 2011.

[25] C. Dilgen, S. Dilgen, D. Fuhrman, O. Sigmund, and B. Lazarov, Topology optimization of turbulent flows, Computer Methods in Applied Mechanics and Engineering, 331 (2018), pp. 363-393.

[26] A. Ern And J.-L. Guermond, Theory and practice of finite elements, vol. 159, Springer Science \& Business Media, 2013.

[27] L. C. Evans And R. F. Gariepy, Measure theory and fine properties of functions, CRC press, 2015.

[28] P. Frey and P. L. George, Mesh generation: application to finite elements, Wiley Online Library, 2000.

[29] M. Giacomini, O. Pantz, and K. Trabelsi, Volumetric expressions of the shape gradient of the compliance in structural shape optimization, arXiv preprint arXiv:1701.05762, (2017).

[30] V. Girault And P.-A. Raviart, Finite element methods for Navier-Stokes equations: theory and algorithms, vol. 5, Springer Science \& Business Media, 2012.

[31] F. Hecht, New development in freefem++, Journal of numerical mathematics, 20 (2012), pp. 251-266.

[32] A. Henrot and M. Pierre, Variation et optimisation de formes: une analyse géométrique, vol. 48, Springer Science \& Business Media, 2006.

[33] R. Hiptmair, A. Paganini, and S. Sargheini, Comparison of approximate shape gradients, BIT Numerical Mathematics, 55 (2015), pp. 459-485.

[34] A. Jameson, Aerodynamic design via control theory, in Recent advances in computational fluid dynamics (Princeton, NJ, 1988), vol. 43 of Lecture Notes in Engrg., Springer, Berlin, 1989, pp. 377-401.

[35] R. Kimmel and J. A. Sethian, Computing geodesic paths on manifolds, Proceedings of the national academy of Sciences, 95 (1998), pp. 8431-8435.

[36] J.-L. Lions and E. Magenes, Non-Homogenous Boundary Value Problems and Applications, vol. 1, ISpringer, New York, 011972.

[37] G. Marck, M. Nemer, And J.-L. Harion, Topology optimization of heat and mass transfer problems: Laminar flow, Numerical Heat Transfer, Part B: Fundamentals, 63 (2013), pp. 508-539.

[38] G. Marck And Y. PRIVAT, On some shape and topology optimization problems in conductive and convective heat transfers, in OPTI 2014, An International Conference on Engineering and Applied Sciences Optimization, M. Papadrakakis, M. Karlaftis, and N. Lagaros, eds., June 2014, pp. 1640-1657.

[39] B. Mohammadi and O. Pironneau, Applied shape optimization for fluids, Numerical Mathematics and Scientific Computation, Oxford University Press, Oxford, second ed., 2010.

[40] F. Murat And J. Simon, Sur le contrôle par un domaine géométrique, rapport la 189, univ, Paris VI, (1976).

[41] S. Osher AND F. SANTosa, Level set methods for optimization problems involving geometry and constraints: I. frequencies of a two-density inhomogeneous drum, Journal of Computational Physics, 171 (2001), pp. 272-288.

[42] S. Osher AND J. A. Sethian, Fronts propagating with curvature-dependent speed: algorithms based on hamilton-jacobi formulations, Journal of computational physics, 79 (1988), pp. 12-49.

[43] O. Pantz, Sensibilité de l'équation de la chaleur aux sauts de conductivité, Comptes Rendus Mathematique, 341 (2005), pp. 333-337.

[44] P. Papazoglou, Topology optimization of heat exchangers, Master's thesis, TU Delft, 2015.

[45] O. Pironneau, On optimum profiles in stokes flow, Journal of Fluid Mechanics, 59 (1973), pp. 117-128.

[46] - Optimal shape design for elliptic systems, Springer Science \& Business Media, 2012.

[47] P. Plotnikov and J. SokoŁowski, Compressible Navier-Stokes equations: theory and shape optimization, vol. 73, Springer Science \& Business Media, 2012.

[48] T. Richter, Fluid-structure interactions, vol. 118 of Lecture Notes in Computational Science and Engineering, Springer, Cham, 2017. Models, analysis and finite elements.

[49] J. A. Sethian, A fast marching level set method for monotonically advancing fronts, Proceedings of the National Academy of Sciences, 93 (1996), pp. 1591-1595.

[50] J. A. Sethian And A. Wiegmann, Structural boundary design via level set and immersed interface methods, Journal of computational physics, 163 (2000), pp. 489-528. 
[51] J. Sokolowski And J.-P. ZolÉsio, Introduction to shape optimization, vol. 16 of Springer Series in Computational Mathematics, Springer-Verlag, Berlin, 1992. Shape sensitivity analysis.

[52] J. Strain, Semi-lagrangian methods for level set equations, Journal of Computational Physics, 151 (1999), pp. $498-533$.

[53] A. Takezawa, S. Nishiwaki, and M. Kitamura, Shape and topology optimization based on the phase field method and sensitivity analysis, Journal of Computational Physics, 229 (2010), pp. 2697-2718.

[54] L. Tartar, An introduction to Sobolev spaces and interpolation spaces, vol. 3, Springer Science \& Business Media, 2007.

[55] R. Temam, Navier stokes equations: Theory and numerical analysis, vol. 45, North-Holland Publishing Company, 1977.

[56] M. Y. WANG, X. WANG, AND D. GuO, A level set method for structural topology optimization, Computer methods in applied mechanics and engineering, 192 (2003), pp. 227-246.

[57] K. Welker, Suitable spaces for shape optimization, arXiv preprint arXiv:1702.07579, (2017).

[58] Q. XIA AND M. Y. WANG, Topology optimization of thermoelastic structures using level set method, Computational Mechanics, 42 (2008), pp. 837-857.

[59] G. H. Yoon, Topological layout design of electro-fluid-thermal-compliant actuator, Computer Methods in Applied Mechanics and Engineering, 209-212 (2012), pp. $28-44$.

[60] - Stress-based topology optimization method for steady-state fluid-structure interaction problems, Computer Methods in Applied Mechanics and Engineering, 278 (2014), pp. 499 - 523.

[61] H. ZHAO, A fast sweeping method for eikonal equations, Mathematics of computation, 74 (2005), pp. 603-627.

[62] X. Zhao, M. Zhou, O. Sigmund, and C. Andreasen, A "poor man's approach" to topology optimization of cooling channels based on a darcy flow model, International Journal of Heat and Mass Transfer, 116 (2018), pp. 1108-1123. 